\title{
Checklist of Epicauta Dejean from America (Meloidae, Meloinae, Epicautini)
}

\author{
María Paula Campos-Soldini', Melania Edith Safenraiter', \\ Leandro Sebastián Wagner', Estrella Natalín Fernández', Christian Javier Sequin' \\ I Laboratorio de Entomología CICyTTP-CONICET/FCYT-UADER, Diamante, Entre Ríos, Argentina \\ Corresponding author: María Paula Campos-Soldini (mariapaulacampos@gmail.com)
}

Academic editor: Warren Steiner | Received 2 January 2018 | Accepted 8 August 2018 | Published 17 December 2018

http://zoobank.org/EA272FFC-02EE-4036-BA2C-1D5D379F1F38

Citation: Campos-Soldini MP, Safenraiter ME, Wagner LS, Fernández EN, Sequin CJ (2018) Checklist of Epicauta Dejean from America (Meloidae, Meloinae, Epicautini). ZooKeys 807: 47-125. https://doi.org/10.3897/ zookeys.807.23375

\begin{abstract}
This paper presents a comprehensive list of American Epicauta. Two hundred and sixty-four named species were recorded in this checklist based primarily on literature and museum records. Seventy-two species were included in the subgenus Macrobasis and 192 species into the nominotypic subgenus. Nomenclatural modifications are provided for E. langei Borchmann and E. nigripes Borchmann, both junior synonyms of E. pilme (Molina); E. albicincta Borchmann, junior synonym of E. suturalis (Haag-Rutenberg); E. lugubris Denier, junior synonym of E. tristis (Mäklin); and E. wagneri Pict, junior synonym of E. vicina (HaagRutenberg). Three hundred and ninety-two new records for South America are provided.
\end{abstract}

\section{Keywords}

blister beetles, Epicautini, new records, review

\section{Introduction}

Reviews of American Epicauta Dejean (1834) were compiled by several authors in different contributions (Werner 1945, 1954, 1955, 1957, 1958, 1973; Kaszab 1953, 1969; Pinto 1975, 1980, 1984, 1999; Admas and Selander 1979; Selander 1982; Selander and Mathieu 1969; Ballmer 1980). The most recent reviews of North and Cen- 
tral America were compiled by Pinto (1999) and García-París et al. (2007) and they listed a total of 173 and 124 respectively. There are no contributions for South America that include Epicauta in a catalogue. The reviews are gathered mostly from works dating to the last century, such as Berg 1881, 1883; Burmeister 1881; Horn 1873; Brèthes 1910, 1915; Borchmann 1917, 1930, 1937, 1940; Denier 1933a, b, 1934, 1935a, b, 1940; Pic 1928; Blackwelder 1945; Werner 1945, 1954, 1955; Martínez 1951, 1952, 1954, 1955, 1958, 1959, 1992; MacSwain 1956; Adams and Selander 1979; Selander 1981a b, 1982, 1984, 1991; Selander and Mathieu 1969; Agafitei and Selander 1980; Kaszab 1959, 1960; 1963a, b; and Pinto 1975, 1980, 1984, 1991. In fact, the South American species are listed in the old catalogue of Blackwelder (1945) which includes others North and Central American Epicauta species. The need to verify and update the checklist of Americas Epicauta became evident after the authors presented numerous new records for South America and saw the need to include all species of Epicauta in a single and updated catalogue.

\section{Materials and methods}

The species of Epicauta were arranged in the order of the reviews of Pinto (1991) and the distribution data were compiled analysing all the available publications and museum data from the following institutions: Museo de La Plata, La Plata, Argentina (MLPA) (see Campos-Soldini et al. 2009), Instituto y Fundación Miguel Lillo, Tucumán, Argentina (IMLA), Museo de Ciencias Naturales "Florentino Ameghino" (MCNFA), and Museo Argentino de Ciencias Naturales 'Bernardino Rivadavia', Buenos Aires, Argentina (MACN), and the private collection of Barriga-Tunón.

For seasonal distribution and information on larval stages see Selander and Weddle (1969), Pinto (1980, 1991), Bologna (1991), and Pinto and Bologna (1999). For identification keys to Macrobasis and the nominotypical subgenus from North and Central America see Pinto (1991). For host plants and identification keys to E. bella group, $E$. vittata group, and E. maculata group from South America see Campos-Soldini (2011), Campos-Soldini and Roig-Juñent (2011), and Campos-Solidni and Roig-Juñent (2015), respectively. Junior synonyms are provided for each species when applicable.

\section{Results}

Two hundred and sixty- four named species are recorded in this checklist based primarily on the literature and museum records. Seventy- two species are included in the subgenus Macrobasis and 192 species into the nominotypic subgenus. Nomenclatural modifications are provided for E. langei Borchmann and E. nigripes Borchmann junior synonym of $E$. pilme (Molina), E. albicincta Borchmann junior synonym of $E$. suturalis (Haag-Rutenberg), E. lugubris Denier junior synonym of E. tristis (Mäklin) and $E$. wagneri Pict junior synonym of E. vicina (Haag-Rutenberg). Three hundred and nine- 
ty-two new records for South America are provided for the following species: E. adspersa (Klug); E. aemula (Fischer); E. albomarginata (Mäklin); E. anthracina (Erichson); E. assimilis (Haag-Rutembreg); E. aterrima (Klug); E. avellanea Denier; E. aymara Denier; E. bosqi Denier; E. bruchi Borchmann; E. brunneipennis (Haag-Rutembreg); E. carmelita (Haag-Rutenberg); E. caustica Rojas; E. clericalis (Berg); E. costipennis Borchmann; E. curticollis Borchmann; E. diagramma (Burmeister); E. excavata (Klug); E. flavogrisea (Burmeisteir); E. floydwerneri Martínez; E. franciscana Denier; E. fulvicornis (Burmeister); E. fumosa (Germar); E. grammica (Fischer); E. griseonigra (Fairmaire); E. hieroglyphica (Haag-Rutenberg); E. inconstants (Fischer); E. koebleri Denier; E. kraussi (Haag-Rutenberg); E. leopardina (Hagg-Rutenberg); E. lizeri Denier; E. luctifera (Fairmaire); E. minutepunctata Borchmann; E. missionum (Berg); E. montei Denier; E. nattereri Haag-Rutenberg; E. nigropunctata Blanchard); E. philaemata (Klug); E. pilme Molina; E. pluvialis Borchmann; E. pullata (Berg); E. purpureiceps (Berg); E. riojana (Fairmaire); E. rutilifrons Borchmann; E. sanguinithorax (Haag-Rutenberg); E. semivittata (Fairmaire); E. subvittata (Erichson); E. suturalis (Haag-Rutenberg); E. talpa (Haag-Rutenberg); E. tristis (Mäklin); E. vicina (Haag-Rutenberg); E. vidua (Klug); E. xanthomera (Fischer); E. xanthocephala (Klug); E. yungana Denier; and E. zebra (Dohrn) (Figs 1-9). Epicauta rutilifrons is the only species with its new records not mapped because it did not have reference points.

\section{Checklist}

\section{Epicautini Denier, 1935}

\section{Epicauta Dejean, 1834}

${ }^{\dagger}$ Epicauta sanctoruensis Zaragoza-Caballero \& Velasco-de León, 2003

\section{Species of Epicauta (Maculata)}

\section{Epicauta (Macrobasis) alastor Skinner, 1904}

Epicauta alastor Skinner, 1904: 217; Werner 1945: 436; 1954: 27; Werner et al. 1966: 46; Selander and Mathieu 1969: 15; Pinto 1991: 69.

Distribution. Mexico, United States.

Location. Mexico: Sinaloa, Sonora. United States: Arizona, California, Texas.

\section{Epicauta (Macrobasis) albida (Say, 1824)}

Lytta albida Say, 1824: 305.

Lytta luteicornis LeConte, 1854: 84.

Cantharis albida: Gemminger and Harold 1870: 2147. 
Macrobasis albida: LeConte, 1863-66: 68; Horn 1873: 89; Snow 1879: 69; Dugès 1889: 57; Champion 1891-93: 397; Chittenden 1903: 26; Fall and Cockerell 1907: 209.

Epicauta albida: Denier 1935: 152; Werner 1945: 511; Blackwelder 1945: 482; Dillon 1952: 403; MacSwain 1956: 55; Parker and Wakeland 1957: 26; Selander and Mathieu 1969: 110; Pinto 1991: 69.

Distribution. Mexico, United States.

Location. Mexico: Coahuila, Nuevo León, Tamaulipas. United States: Arizona, Colorado, Kansas, Nebraska, New Mexico, Oklahoma, Texas, Wyoming.

Epicauta (Macrobasis) alpina Werner, 1944

Epicauta alpina Werner, 1944: 67; Werner et al. 1966: 49; Pinto 1991: 80.

Distribution. United States.

Location. United States: Arizona, New Mexico, Texas.

Epicauta (Macrobasis) apicalis Dugès, 1889

Epicauta apicalis Dugès, 1889: 410; Denier 1935: 152; Blackwelder 1945: 482; Pinto 1991: 75.

Distribution. Mexico.

Location. Mexico: Nayarit.

Epicauta (Macrobasis) arizonica Werner, 1944

Epicauta arizonica Werner, 1944: 72; 1966: 48; Selander 1982: 797; Pinto 1991: 71.

Distribution. Mexico, United States.

Location. Mexico: Baja California Sur, Chihuahua, Nayarit, Sonora. United States: Arizona.

Epicauta (Macrobasis) atricolor Champion, 1892

Epicauta atricolor Champion, 1892: 419; Denier 1935: 152; Blackwelder 1945: 482;

Werner 1954: 27; 1958: 1; Pinto 1982: 405; 1991: 80; 1991: 80.

Distribution. Mexico.

Location. Mexico: Morelos, Oaxaca, Veracruz.

Epicauta (Macrobasis) atripilis Champion, 1892

Epicauta atripilis Champion, 1892: 410; Denier 1935: 152; Blackwelder 1945: 482; Pinto 1991: 75.

Distribution. Mexico. 
Location. Mexico: Oaxaca, Veracruz.

Epicauta (Macrobasis) atrivittata (LeConte, 1854)

Lytta atrivittata LeConte, 1854: 224.

Macrobasis atrivittis [sic]: LeConte 1863-66: 68 (lapsus calami).

Cantharis atrivittata: Gemminger and Harlod 1870: 2148.

Macrobasis atrivittata: Horn 1873: 90; Snow 1907: 186; Fall and Cockerell 1907: 209.

Epicauta atrivittata: Denier 1935: 152; Selander and Mathieu 1969: 88; Pinto 1991: 70.

Distribution. Mexico, United States.

Location. Mexico: Chihuahua, Coahuila, Durango. United States: Arizona, New Mexico, Texas.

\section{Epicauta (Macrobasis) balli Werner, 1945}

Epicauta balli Werner, 1945: 460; Werner et al. 1966: 34; Pinto 1991: 81.

Distribution. United States.

Location. United States: Arizona.

Epicauta (Macrobasis) bekeri (Dugès, 1889)

Epicauta bekeri Dugès, 1889: 113.

Macrobasis bekeri: Champion 1892: 400.

Epicauta bekeri: Denier 1935: 153; Blackwelder 1945: 482; Werner 1949: 76; Pinto 1991: 72.

Distribution. Mexico.

Location. Mexico: Colima, Durango, Morelos, Nayarit.

Epicauta (Macrobasis) bipunctata Werner, 1958

Epicauta bipunctata Werner, 1958: 7; Selander and Ahafitei 1982: 200; Pinto 1991: 81.

Distribution. Mexico.

Location. Mexico: Guerrero, Jalisco, Morelos.

Epicauta (Macrobasis) borrei Werner, 1958

Lytta fumosa Haag-Rutenberg, 1880: 40, nec Germar, 1824.

Cantharis borrei Dugès, 1881: 145.

Macrobasis borrei Horn, 1885: 107.

Macrobasis fumosa: Champion 1899: 178.

Epicauta borrei: Denier 1935: 153; Blackwelder 1945: 482; Pinto 1991: 81.

Distribution. Mexico. 
Location. Mexico: Aguascalientes, Durango, Guanajuato, Jalisco, Mexico, Michoacán, Zacatecas.

Epicauta (Macrobasis) candezi (Haag-Rutenberg, 1880)

Lytta candezi Haag-Rutenberg, 1880: 43.

Epicauta diversicornis: Champion 1892: 399 (in part).

Epicauta candezi: Werner 1949: 76; Pinto 1991: 72.

Distribution. El Salvador, Guatemala, Mexico.

Location. El Salvador and Guatemala: country labeled only. Mexico: Chiapas, Yucatán.

Epicauta (Macrobasis) cinereiventris Champion, 1892

Epicauta cinireiventris Champion, 1892: 411; Denier 1935: 154; Blackwelder 1945: 483; Pinto 1991: 75.

Distribution. Mexico.

Location. Mexico: Chiapas, Guerrero.

\section{Epicauta (Macrobasis) croceicincta Dugès, 1881}

Cantharis croceicincta Dugès, 1881: 143; Champion 1892: 42.

Epicauta croceicincta: Denier 1935: 154; Blackwelder 1945: 483; Werner 1954: 27;

Pinto 1991: 75.

Distribution. Mexico.

Location. Mexico: Guanajuato, Jalisco.

Epicauta (Macrobasis) disparilis (Champion, 1892)

Macrobasis disparilis Champion, 1892: 398.

Epicauta disparilis: Denier 1935: 154; Blackwelder 1945: 483; Werner 1973: 463;

Pinto 1991: 78.

Distribution. Mexico.

Location. Mexico: Guerrero, Puebla.

Epicauta (Macrobasis) distorta (Champion, 1892)

Macrobasis distorta Champion, 1892: 396.

Epicauta distorta: Denier 1935: 154; Blackwelder 1945: 483; Werner 1973: 463; Pinto 1991: 79.

Distribution. Costa Rica, Honduras, Mexico, Nicaragua.

Location. Mexico: Guerrero. 
Epicauta (Macrobasis) diversicornis (Haag-Rutenberg, 1880)

Lytta diversicornis Haag-Rutenberg, 1880: 42.

Lytta pallida Haag-Rutenberg, 1880: 42.

Macrobasis flavens Dugès, 1889: 58.

Epicauta diversicornis: Denier 1935: 154; Blackwelder 1945: 483; Werner 1949: 76;

Pinto 1991: 72.

Distribution. Mexico.

Location. Mexico: Guerrero, Hidalgo, Jalisco, Mexico, Michoacán, Morelos, Sinaloa, Sonora.

Epicauta (Macrobasis) dohrni (Haag-Rutenberg, 1880)

Lytta dohrni Haag-Rutenberg, 1880: 45.

Lytta bimaculosa Kirsch, 1886: 336.

Lytta bogotensis Pic, 1916: 9.

Epicauta dohrni: Champion 1892: 409; Denier 1935: 22; Blackwelder 1945: 483;

Pinto 1991: 73.

Distribution. Colombia, Panama: country labeled only.

Epicauta (Macrobasis) evanescens Champion, 1892

Epicauta evanescens Champion, 1892: 412; Denier 1935: 155; Blackwelder 1945: 483;

Pinto 1991: 76.

Distribution. Guatemala, Mexico.

Location. Guatemala: country labeled only. Mexico: Chiapas.

Epicauta (Macrobasis) excors (Fall, 1909)

Macrobasis excors Fall, 1909: 166.

Epicauta excors: Denier 1935: 155; Werner 1945: 503; Pinto 1991: 79.

Distribution. Mexico.

Location. Mexico: Baja California Sur.

Epicauta (Macrobasis) fabricii (LeConte, 1853)

Lytta cinerea Fabricius, 1798: 119, nec Meloe cinereous Forster, 1771.

Lytta fabricii LeConte, 1853: 343.

Lytta debilis LeConte, 1853: 344.

Cantharis fabricii: Gemminger and Harold 1980: 2150.

Epicauta murinoides Dillon, 1952: 409.

Epicauta fabricii: Werner et al. 1966: 46; Selander 1982: 820; Pinto 1991: 74. 
Distribution. Canada, United States.

Location. Canada: Manitoba. United States: Arizona, Colorado, Kansas, Montana, Nebraska, North Dakota, Oklahoma, South Dakota, Texas, Utah.

Epicauta (Macrobasis) flagellaria (Erichson, 1848)

Lytta flagellaria Erichson, 1848: 566.

Cantharis flagellaria: Gemminger and Harold 1870: 2150.

Lytta intermedia Haag-Rutenberg, 1880: 56.

Epicauta intermedia: Blackwelder 1945: 483.

Epicauta flagellaria: Blackwelder 1945: 483; Werner 1949: 76; Pinto 1991: 72.

Distribution. Colombia: Barranquilla, Tolima, Panama, Trinidad, Venezuela.

\section{Epicauta (Macrobasis) flavocinerea (Blatchely, 1910)}

Macrobasis flavocinereus Blatchely, 1910: 1359.

Epicauta flavocinerea: Denier 1935: 155; Werner 1945: 499; Pinto 1991: 74.

Distribution. Canada, United States.

Location. Canada: New Brunswick. United States: Colorado, Iowa, Maine, Massachusetts, Michigan, Minnesota, Montana, Nebraska, New Hampshire, North Dakota, South Dakota, Wisconsin, Wyoming.

Epicauta (Macrobasis) forticornis (Haag-Rutenberg, 1880)

Lytta forticornis Haag-Rutembeerg, 1880: 41.

Epicauta forticornis: Denier 1935: 155; Blackwelder 1945: 483; Werner 1949: 75;

Pinto 1991: 72.

Distribution. Mexico.

Location. Mexico: Guerrero, Morelos, Nayarit, Yucatán.

Epicauta (Macrobasis) funesta (Chevrolat, 1834)

Lytta funesta Chevrolat, 1834: 3.

Cantharis funesta: Gemminger and Harold 1870: 2150.

Epicauta funesta: Champion 1892: 410; Denier 1935: 155; Blackwelder 1945: 483;

Selander and Agafitei 1982: 200; Pinto 1991: 76.

Distribution. Mexico.

Location. Mexico: Puebla, Veracruz.

Epicauta (Macrobasis) gissleri (Horn, 1878)

Macrobasis gissleri Horn, 1878: 58.

Epicauta gissleri: Denier 1935: 155; Werner 1945: 496; Werner et al. 1966: 52; Pinto 1991: 78 
Distribution. United States.

Location. United States: Arizona, New Mexico, Texas.

Epicauta (Macrobasis) haroldi (Haag-Rutenberg, 1880)

Lytta haroldi Haag-Rutenberg, 1880: 44.

Epicauta haroldi: Champion 1892: 410; Denier 1935: 156; Blackwelder 1945: 483;

Werner 1958: 1; Pinto 1991: 76.

Distribution. Costa Rica, Guatemala.

Epicauta (Macrobasis) hirsutipubescens (Maydell, 1934)

Macrobasis hirustipubescens Maydell, 1934: 334.

Epicauta virgulata: Werner 1945: 512 (in part).

Epicauta hirustipubescens: Denier 1940: 420; Werner 1949: 110; Werner et al. 1966:

51; Pinto 1991: 83.

Distribution. Mexico, United States.

Location. Mexico: Chihuahua, Coahulia, Durango, Sonora. United States: Arizona, New Mexico, Texas.

\section{Epicauta (Macrobasis) bumeralis (Dugès, 1889)}

Macrobasis humeralis Dugès, 1889: 58; Champion, 1892: 400.

Epicauta humeralis: Denier 1935: 156; Werner 1949: 76; Pinto 1991: 72.

Distribution. Mexico.

Location. Mexico: Morelos, Jalisco, Nayarit.

\section{Epicauta (Macrobasis) immaculata (Say, 1824)}

Lytta immaculate Say, 1824: 304.

Lytta articularis Say, 1824: 304.

Lytta fulvescens LeConte, 1854: 447.

Cantharis fulvescens: Gemminger and Harold 1870: 2150.

Cantharis immaculata: Gemminger and Harold 1870: 2151.

Macrobasis fulvescens: LeConte 1863-66: 68; Snow 1879: 69.

Macrobasis immaculate: LeConte 1863-66: 68; Horn 1873: 93; Ulke 1875: 862; Chittenden 1903: 26; Blatchley 1910: 1359; Milliken 1921: 7; Hatch and Ortenburger 1930: 13; Böving and Craighead 1931: 96; Carruth 1931: 53; Gilberston and Horsfall 1940: 5; Montgomery and Amos 1941: 254; Schwitzgebel and Wilbur 1942: 42.

Cantharis basalis Dugès, 1881: 144; 1889: 71.

Macrobasis basalis: Champion 1892: 402.

Epicauta immaculata: Denier 1935: 156; Werner 1945: 489; Dillon 1952: 407; MacSwain 1956: 52; Selander and Mathieu 1969: 113; Pinto 1991: 70. 
Distribution. Mexico, United States.

Location. Mexico: Coahuila, Tamaulipas, Veracruz. United States: Alabama, Arkansas, Colorado, Georgia, Illinois, Indiana, Iowa, Kansas, Kentucky, Louisiana, Mississippi, Missouri, Nebraska, New Mexico, Ohio, Oklahoma, South Dakota, Tennessee, Texas, West Virginia.

Epicauta (Macrobasis) ingrata Fall, 1907

Epicauta ingrata Fall, 1907: 258; Denier 1935: 156; Werner et al. 1966: 45; Pinto 1991: 77

Distribution. United States.

Location. United States: Arizona, Colorado, New Mexico.

\section{Epicauta (Macrobasis) isthmica Werner, 1949}

Epicauta isthmica Werner, 1949: 72; Pinto 1991: 73.

Distribution. Belize, Costa Rica, Honduras, Mexico, Nicaragua, Panama.

Location. Belize, Costa Rica, Hondura, Nicaragua, and Panama. Mexico: Querétaro, Veracruz.

\section{Epicauta (Macrobasis) labialis (Dugès, 1881)}

Cantharis labialis Dugès, 1881: 145.

Macrobasis labialis: Dugès 1889: 86.

Ganospasta labialis: Champion 1892: 403.

Epicauta labialis: Selander 1954: 86; Werner 1958: 19; Pinto 1991: 81.

Distribution. Mexico.

Location. Mexico: Guanajuato, Jalisco.

\section{Epicauta (Macrobasis) languida (Horn, 1895)}

Macrobasis languida Horn, 1895: 252.

Epicauta languida: Denier 1935: 156; Blackwelder 1945: 483; Werner 1945: 501;

1954: 113; Pinto 1991: 90.

Distribution. Mexico.

Location. Mexico: Baja California Sur.

\section{Epicauta (Macrobasis) lauta (Horn, 1885)}

Macrobasis lauta Horn, 1885: 108.

Epicauta compressicollis Champion, 1892: 427.

Epicauta macroflexi Dillon, 1952: 414.

Epicauta lauta: Denier 1935: 156; Vaurie 1950: 86; Werner 1954: 27; Werner et al.

1966: 47; Selander 1982: 797; Pinto 1991: 78. 
Epicauta lauta rossi Werner, 1949: 108.

Epicauta lauta lauta: Selander 1954: 86.

Distribution. Mexico, United States.

Location. Mexico: Baja California Sur, Chihuahua, Coahuila, Durango. United States: Arizona, California, New Mexico, Texas.

\section{Epicauta (Macrobasis) leoni Dugès, 1889}

Epicauta leoni Dugès, 1889: 15; Denier 1935: 156; Blackwelder 1945: 483; Werner 1958: 15; Selander and Agafitei 1982: 200; Pinto 1991: 82.

Distribution. Mexico.

Location. Mexico: Michoacán, Nuevo León, San Luis Potosí, Tamaulipas.

Epicauta (Macrobasis) liebecki Werner, 1944

Epicauta liebecki Werner, 1944: 72; Werner et al. 1966: 49; Pinto 1991: 73.

Distribution. United States.

Location. United States: Arizona.

Epicauta (Macrobasis) linearis (LeConte, 1858)

Lytta linearis LeConte, 1858: 23.

Epicauta linearis: Denier 1935: 156; Werner 1945: 513; Selander 1954: 86; Werner et al. 1966: 56; Pinto 1991: 83.

Distribution. Mexico, United States.

Location. Mexico: Chihuahua, Durango. United States: Arizona, New Mexico, Texas.

Epicauta (Macrobasis) longicollis (LeConte, 1853)

Lytta longicollis LeConte, 1853: 343.

Macrobasis longicollis: LeConte 1863-66: 68; Horn 1873: 90; Champion 1891-93: 397;

Snow 1906: 174; Fall and Cockerell 1907: 209; Cockerell and Harris 1925: 31.

Cantharis longicollis: Gemminger and Harold 1870: 2151.

Epicauta longicollis: Denier 1935: 156; 1940: 421; Blackwelder 1945: 483; Werner

1945: 508; Vaurie 1950: 35; Dillon 1952: 406; MacSwain 1956: 56; Selander and Mathieu 1969: 109; Pinto 1991: 71.

Distribution. Mexico. United States.

Location. Mexico: Chihuahua, Coahuila. United States: Arizona, Colorado, Missouri, New Mexico, Texas.

Epicauta (Macrobasis) maculifera (Maydell, 1934)

Macrobasis maculifera Maydell, 1934: 335. 
Epicauta maculifera: Denier 1940: 421; Werner 1945: 514; 1973: 463; Werner et al. 1966: 50; Pinto 1991: 79.

Distribution. United States.

Location. United States: Arizona.

Epicauta (Macrobasis) melanochroa Wellman, 1910

Cantharis nigra Dugès, 1870: 161.

Epicauta nigra: Dugès 1889: 76, nec Woodhouse 1800.

Epicauta melanochroa Wellman, 1910: 24 (n. repl. name); Werner 1958: 11; Pinto 1991: 82

Distribution. Mexico.

Location. Mexico: Guanajuato, Jalisco, Mexico, Michoacán, Morelos, Nayarit, Querétaro.

Epicauta (Macrobasis) mimetica (Horn, 1875)

Cantharis mimetica Horn, 1875: 154.

Epicauta mimetica: Werner 1945: 453; 1958: 17.

Distribution. United States.

Location. United States: Texas.

Epicauta (Macrobasis) murina (LeConte, 1853)

Cantharis unicolor Kirby, 1837: 241, nec Cantharis unicolor Faldermann, 1835.

Lytta murina LeConte, 1853: 344.

Cantharis murina: Gemminger and Harold 1870: 2152.

Epicauta murina: Werner 1945: 499; Pinto 1991: 74.

Distribution. Canada, United States.

Location. Canada: New Brunswick. United States: Colorado, Iowa, Maine, Massachusetts, Michigan, Minnesota, Montana, Nebraska, New Hampshire, North Dakota, South Dakota, Wisconsin, Wyoming.

Epicauta (Macrobasis) nigritibialis Werner, 1958

Epicauta nigritibialis Werner, 1958: 5; Pinto 1991: 82.

Distribution. Mexico.

Location. Mexico: Coahuila.

Epicauta (Macrobasis) niveolineata (Haag-Rutenberg, 1880)

Lytta niveolineata Haag-Rutenberg, 1880: 46.

Epicauta niveolineata: Denier 1935: 158; Blackwelder 1945: 483; Werner 1958: 4;

Selander and Agafitei 1982: 200; Pinto 1991: 82. 
Distribution. Mexico.

Location. Mexico: Chiapas, Guerrero, Oaxaca, Veracruz.

Epicauta (Macrobasis) ochrea (LeConte, 1853)

Lytta ochrea LeConte, 1853: 342.

Cantharis ochrea: Gemminger and Harold 1870: 2152.

Cantharis protarsalis Dugès, 1877: 62.

Macrobasis ochrea: Champion 1892: 401.

Epcicauta monoliformis Dillon, 1950: 103.

Epicauta ochrea: Denier 1935: 158; Blackwelder 1945: 484; Werner 1945: 495; 1954:

106; Werner et al. 1966: 52; Pinto 1991: 78.

Distribution. Mexico, United States.

Location. Mexico: Guanajuato, Sonora. United States: Arizona, Oklahoma, Texas, Utah.

Epicauta (Macrobasis) pacifica Maydell, 1934

Epicauta pacifica Maydell, 1934: 330; Denier 1940: 421; Blackwelder 1945: 484; Pinto 1991: 76.

Distribution. Mexico.

Location. Mexico: Jalisco.

Epicauta (Macrobasis) parkeri Werner, 1944

Epicauta parkeri Werner, 1944: 71; 1945: 497; Werner et al. 1966: 53; Pinto 1991: 78.

Distribution. United States.

Location. United States: Arizona, Colorado, New Mexico.

Epicauta (Macrobasis) polingi Werner, 1944

Epicauta polingi Werner, 1944: 71; Werner et al. 1966: 48; Selander 1982: 804; Pinto 1991: 73.

Epicauta luteola Dillon, 1952: 411.

Distribution. Mexico, United States.

Location. Mexico: Chihuahua, Coahuila, Durango, Nuevo León, Tamaulipas. United States: Arizona, Texas.

Epicauta (Macrobasis) prosopidis Werner, 1973

Epicauta prosopidis Werner, 1973: 461; Pinto 1991: 79.

Distribution. Mexico.

Location. Mexico: Durango. 
Epicauta (Macrobasis) punctum (Dugès, 1870)

Cantharis punctum Dugès, 1870: 158.

Epicauta punctum: Champion 1892: 410; Blackwelder 1945: 484; Pinto 1991: 76.

Distribution. Mexico.

Location. Mexico: Morelos, Oaxaca.

\section{Epicauta (Macrobasis) purpurea (Horn, 1885)}

Macrobasis purpurea Horn, 1885: 108.

Epicauta purpurea: Werner 1945: 504; 1973: 463; Werner et al. 1966: 50; Pinto 1991:

79.

Distribution. Mexico, United States.

Location. Mexico: Durango, Sinaloa, Sonora. United States: Arizona.

\section{Epicauta (Macrobasis) segmenta (LeConte, 1853)}

Lytta segmenta LeConte, 1853: 343.

Apterospasta segmentata: LeConte 1862: 272; 1863-66: 68.

Macrobasis segmenta: Horn 1873: 93; Champion 1891-93: 401; Chittenden 1903: 26;

Snow 1906: 174; 1907: 150; Carruth 1931: 54; Whelan 1939: 118.

Macrobasis cinctothorax Dugès, 1889: 65.

Epicauta segmenta: Blackwelder 1945: 484; Selander and Mathieu 1969: 116; Pinto 1991: 71.

Distribution. Mexico, United States.

Location. Mexico: Chihuahua, Coahuila, Durango, Sinaloa, Sonora. United States: Arizona, California, Kansas, Nebraska, New Mexico, Oklahoma, South Dakota, Texas, Wyoming.

\section{Epicauta (Macrobasis) selanderorum Werner, 1958}

Epicauta selanderorum Werner, 1958: 6; Pinto 1991: 82.

Distribution. Mexico.

Location. Mexico: Jalisco, Michoacán, Querétaro.

Epicauta (Macrobasis) stigmata (Dugès, 1870)

Cantharis stigmata Dugès, 1870: 159.

Lytta neglecta Haag-Rutenberg, 1880: 54.

Epicauta stigmata: Denier 1935: 159; Blackwelder 1945: 484; Werner 1958: 9; Pinto 1982, Pinto 1991: 82.

Distribution. Mexico.

Location. Mexico: Guanajuato, Jalisco, Mexico, Michoacán, Morelos, Querétaros, Puebla, Tlaxcala. 
Epicauta (Macrobasis) subglabra (Fall, 1922)

Macrobasis subglabra Fall, 1922: 173.

Epicauta subglabra: Denier 1935: 159; Werner et al. 1966: 47; Pinto 1991: 74.

Distribution. Canada, United States.

Location. Canada: Alberta, Manitoba, Saskatchewan. United States: Arizona, Idaho, Michigan, New Mexico, North Dakota, South Dakota.

Epicauta (Macrobasis) sublineata (LeConte, 1854)

Lytta sublineata LeConte, 1854: 447.

Cantharis sublineata: Gemminger and Harold 1870: 2154.

Macrobasis sublineata: LeConte 1863-66: 68; Horn 1873: 94.

Macrobasis megacephala Champion, 1892: 402.

Epicauta reinhardi Dillon, 1952: 413.

Epicauta sublineata: Selander and Mathieu 1969: 116; Pinto 1991: 71.

Distribution. Mexico, United States.

Location. Mexico: Coahuila, Nuevo León, Tamaulipas. United States: Texas.

Epicauta (Macrobasis) tenella (LeConte, 1854)

Lytta tenella LeConte, 1854: 23.

Cantharis tenella: Gemminger and Harold 1870: 2154.

Epicauta merkeliana Horn, 1891: 43.

Epicauta tenella: Horn 1891: 43; Denier 1935: 159; Selander 1954: 87; Werner et al. 1966: 45; Pinto 1991: 79.

Distribution. Mexico, United States.

Location. Mexico: Baja California, Chihuahua, Durango, Sonora. United States: Arizona, California, New Mexico, Nevada, Texas.

Epicauta (Macrobasis) tenuemarginata Werner, 1958

Epicauta tenuemarginata Werner, 1958: 13; Pinto 1991: 83.

Distribution. Mexico.

Location. Mexico: Jalisco, Michoacán.

Epicauta (Macrobasis) tenuicornis (Champion, 1892)

Macrobasis tenuicornis Champion, 1892: 400.

Epicauta tenuicornis: Werner 1954: 27; Pinto 1991: 73.

Distribution. Mexico.

Location. Mexico: Guerrero, Michoacan, Morelos, Puebla. 
Epicauta (Macrobasis) tenuilineata (Horn, 1894)

Macrobasis tenuilineata Horn, 1894: 436.

Epicauta tenuilineata: Denier 1935: 159; Blackwelder 1945: 484; Werner 1945: 502;

Werner et al. 1966: 35; Pinto 1991: 80.

Distribution. Mexico, United States.

Location. Mexico: Baja California Sur. United States: Arizona, California.

Epicauta (Macrobasis) tenuis (LeConte, 1853)

Lytta tenuis LeConte, 1853: 343.

Cantharis tenuis: Gemminger and Harold 1870: 2154.

Epicauta tenuis: Denier 1935: 159; 1940: 422; Werner 1945; Pinto 1991: 80.

Distribution. United States.

Location. United States: Florida, Georgia, South Carolina.

Epicauta (Macrobasis) terminata (Dugès, 1870)

Cantharis terminata Dugès, 1870: 157; Gemminger and Harold 1870: 2154.

Epicauta terminata: Dugès 1889: 78; Champion 1892: 409; Denier 1935: 159; Blackwelder 1945: 484; Selander 1954: 88; Pinto 1991: 77.

Distribution. Mexico.

Location. Mexico: Colima, Guerrero, Jalisco, Michoacán, Oaxaca, Puebla, Veracruz.

\section{Epicauta (Macrobasis) texana Werner, 1944}

Epicauta texana Werner, 1944: 73; Selander and Mathieu 1969: 110; Pinto 1991: 71.

Distribution. Mexico, United States.

Location. Mexico: Durango. United States: Arizona, New Mexico, Texas.

Epicauta (Macrobasis) torsa (LeConte, 1853)

Lytta torsa LeConte, 1853: 343.

Epicauta torsa: Denier 1935: 159; 1940: 422; Werner 1945: 508; 1973: 463; Arnold 1976: 28; Pinto 1991: 79.

Distribution. United States.

Location. United States: Alabama, Florida, Massachusetts, Mississippi, North California, Oklahoma, Texas.

Epicauta (Macrobasis) tripartita Champion, 1892

Epicauta tripartita Champion, 1892: 421; Denier 1935: 159; Blackwelder 1945: 484;

Vaurie 1950: 25; Werner 1958: 4; Pinto 1991: 83. 
Distribution. Mexico.

Location. Mexico: Chihuahua, Nayarit, Sinaloa.

\section{Epicauta (Macrobasis) triquetra Werner, 1958}

Epicauta triquetra Werner, 1858: 15; Pinto 1991: 83.

Distribution. Mexico.

Location. Mexico: San Luis Potosí.

\section{Epicauta (Macrobasis) unicalcarata Champion, 1892}

Epicauta unicalcarata Champion, 1892: 412; Denier 1935: 160; Blackwelder 1945: 484; Pinto 1982: 406; Pinto 1991: 77.

Distribution. Mexico.

Location. Mexico: Guerrero.

\section{Epicauta (Macrobasis) uniforma Werner, 1944}

Epicauta uniforma Werner, 1944: 67; 1945: 452; 1958: 5; Werner et al. 1966: 49;

Selander and Agafitei 1982: 138; 1982: 201; Pinto 1991: 83.

Distribution. Mexico, United States.

Location. Mexico: Chihuahua, Durango, Sonora, Zacatecas. United States: Arizona, Colorado, New Mexico, Texas.

\section{Epicauta (Macrobasis) valida (LeConte, 1853)}

Lytta segmentata LeConte, 1853: 342 (in part).

Lytta valida LeConte, 1858: 39.

Apterospasta valida: LeConte 1862: 272; 1863-66: 68.

Macrobasis segmentata: Horn 1873: 93 (in part); Carruth 1931: 54 (in part).

Epicauta segmenta: Werner 1945: 490 (in part).

Epicauta valida: Selander \& Mathieu, 1969: 118; Pinto 1991: 71.

Distribution. United States.

Location. United States: Colorado, Kansas, Louisiana, Nebraska, New Mexico, Oklahoma.

\section{Epicauta (Macrobasis) virgulata (LeConte, 1866)}

Macrobasis virgulata LeConte, 1866: 156.

Epicauta virgulata: Denier 1935: 169; Werner 1945: 512 (in part); 1949: 108; Werner et al. 1966: 51; Pinto 1991: 83.

Distribution. Mexico, United States.

Location. Mexico: Baja California Norte, Baja California Sur, Sinaloa, Sonora. United States: Arizona, California. 


\section{Species of Epicauta (Epicauta)}

\section{Epicauta (Epicauta) abadona Skinner, 1904}

Epicauta abadona Skinner, 1904: 217; Denier 1935: 152; Werner et al. 1966: 37; Adams and Selander 1979: 249; Pinto 1991: 347.

Epicauta abadona: Maydell 1934: 331 (lapsus).

Epicauta mutchleri Maydell, 1934: 331.

Terra typica. E. abadona, Holotype male, Phoenix, Arizona; ANSP. E. mutchleri, Holotype male, Phoenix, Arizona; AMNH.

Distribution. Mexico, United States.

Location. Mexico: Sinaloa, Sonora. United States: Arizona.

\section{Epicauta (Epicauta) abeona Pinto, 1980}

Epicauta abeona Pinto, 1980: 69; 1991: 272.

Terra typica. Holotype male, Ixtlán del Río, Nayarit; LACM.

Distribution. Mexico.

Location. Mexico: Aguascalientes, Distrito Federal, Jalisco, Mexico, Nayarit.

\section{Epicauta (Epicauta) adspersa (Klug, 1825)}

Lytta adspersa Klug, 1825: 434.

Epicauta conspersa Curtis, 1845: 472.

Cantharis adspersa Burmeister, 1881: 29; Berg 1881: 307; Gemminger and Harold 1870: 2147.

Epicauta adspersa: Bruch 1914: 403; Borchmann 1917: 70; Denier 1935b: 152; Bosq 1943: 10; Blackwelder 1945: 482; Di Iorio 2004: 165; Campos-Soldini and RoigJuñent 2015: 20; Pinto and Bologna 2016: 205.

Terra typica. one Type (s?) Mendoza, Argentina; MLPA location unknown.

Distribution. Argentina, Brazil, Peru, Uruguay.

Location. Argentina: Buenos Aires, Córdoba, Chubut, Entre Ríos, La Pampa, La Rioja, Mendoza, Neuquén, Río Negro, Salta, San Juan, Santa Fe, Santiago del Estero, Tucumán. Brazil. Peru: Junin. Uruguay: Montevideo.

New records (Figure1): Argentina: Buenos Aires (Flores, La Plata, Las Martinetas, San Nicolás, Tandil); Chubut (Trelew); Córdoba (Calamuchita, El Sauce, Río IV, Salsacate, Villa María); Entre Ríos (Concordia, Diamante, Federación, Gualeguaychú, Villaguay) La Pampa (General Pico); La Rioja (Nonogasta, Villa Unión); Mendoza (Cacheuta, Los Pejecitos, Puesto la Obligación, San Rafael, Santa Rosa, Uspallata); Neuquén (Zapala); Río Negro (Choele Cheol); Santa Fe (Reconquista, Rosario); San Juan (Caligasta, Valle Fértil, Villa Nueva, Zonda). Brazil: Mato Grosso (states labeled only). Uruguay: Montevideo (Canelones, Maldonado, Minas, Peńarol, Piriapolis, Punta del Este) [MLPA, Barriga-Tunón, IADIZA, FIMLA, MACN]. 


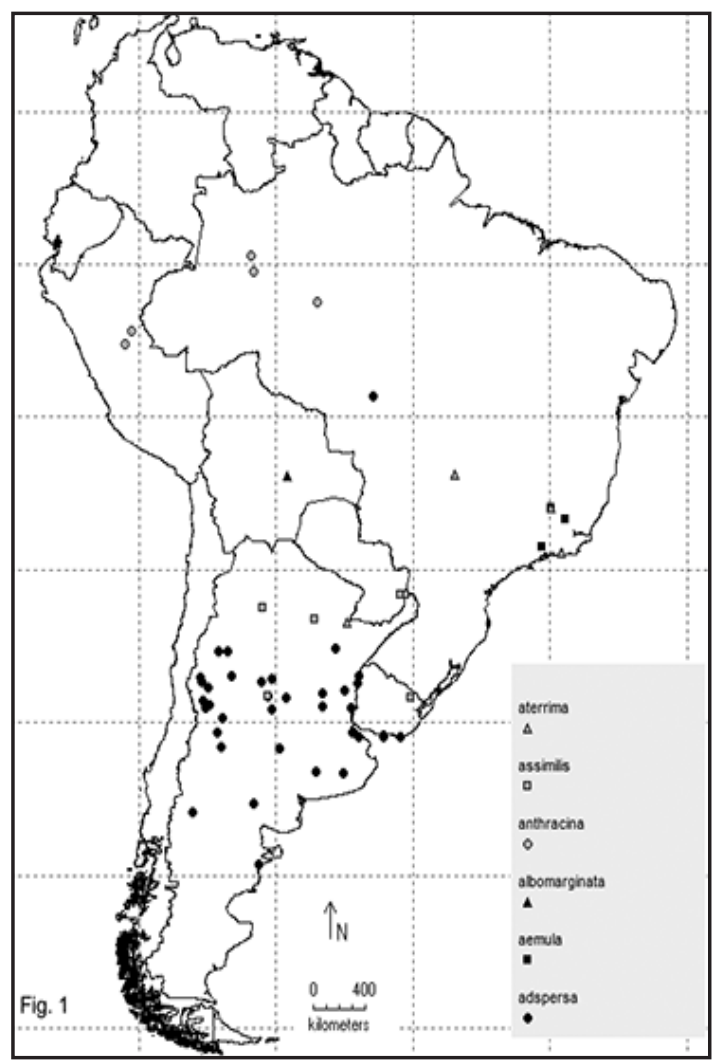

Figure I. New distribution records for: E. adspersa; E. aemula; E. albomarginata; E. anthracina; E. assimilis; and E. aterrima.

\section{Epicauta (Epicauta) aemula (Fischer, 1827)}

Cantharis aemula Fischer, 1827: 20; Gemminger and Harold 1870: 2147.

Lytta aemula Hagg-Rutenberg, 1880: 53.

Epicauta aemula: Borchmann 1917: 70; Denier 1935: 152; Blackwelder 1945: 482;

Adams and Selander 1979: 255.

Terra typica. Type (s?) Brazil; location unknown.

Distribution. Bolivia, Brazil.

Location. Bolivia: Coroico. Brazil: São Paulo.

New records (Figure 1): Brazil: Minas Gerais (Viçosa); Bello Horizonte (Minas); Rio de Janerio (Itatiaia) [MLPA].

\section{Epicauta (Epicauta) afoveata Werner, 1949}

Epicauta afoveata Werner, 1949: 103; 1955: 5; Pinto 1972: 256; 1991: 135.

Terra typica. Holotype male, Borrego, California; MCZC. 
Distribution. Mexico, United States.

Location. Mexico: Baja California Norte, Cedros Island. United States: California.

\section{Epicauta (Epicauta) albolineata (Dugès, 1877)}

Cantharis albolineata Dugès, 1877: 64; Vázquez and Zaragoza 1979: 579.

Epicauta albolineata: Dugès 1889: 84; Champion 1892: 416; Denier 1935: 152; Blackwelder 1945: 482; Werner 1945: 451; Kaszab 1960: 285; Werner et al. 1966: 23; Pinto 1991: 114.

Epicauta duplicata Casey, 1891: 172.

Epicauta costaricensis Kaszab, 1960: 284.

Terra typica. Holotype male, Tupataro, Guanajuato, Mexico; UNAM.

Distribution. Costa Rica, Guatemala, Mexico, United States.

Location. Costa Rica: Belvedero, Santa Elena. Guatemala: Purulha. Mexico: Aguascaliente, Colima, Guanajuato, Guerrero, Jalisco, Mexico, Michoacán, Morelos, Nayarit, San Luis Potosí, Sonora. United States: Arizona.

\section{Epicauta (Epicauta) albomarginata (Mäklin, 1875)}

Cantharis albomarginata Mäklin, 1875: 625.

Epicauta albomarginata: Denier 1935: 152; Denier 1940: 419; Blackwelder 1945: 482. Epicauta denieri Pic, 1933: 26; Denier 1935: 152.

Epicauta limbaticollis Pic, 1924: 2; Denier 1935: 152.

Terra typica. Unknown.

Distribution. Bolivia, Paraguay, Peru.

Location. Bolivia: Lagunillas, Santa Cruz. Paraguay: San Carlos, Río Apa, between Punta Apa and Vella Vista; Santa Cruz. Peru.

New records (Figure 1): Bolivia: Santa Cruz (departament labeled only) [MLPA].

\section{Epicauta (Epicauta) alphonsii Horn, 1874}

Epicauta Maura LeConte, 1851: 162; Horn 1873: 97; Fall 1901: 184; Moore 1937: 42. Lytta Maura: LeConte 1853: 339.

Epicauta alphonsii Horn, 1874: 364; Denier 1935: 152; Pinto 1991: 138.

Epicauta punticollis: MacSwain 1943: 364.

Epicauta californica Werner, 1945: 479 (n. repl. name for E. Maura LeC, 1851, nec

Faldeman, 1833); Werner 1955: 3; Middlekauf 1958: 9, 10; Pinto 1972: 256;

Doyen and Opler 1973: 308.

Terra typica. E. maura LeConte, lectotype male (designated by Werner 1945), California; MCZC. E. alphonsii Horn holotype female, Mariposa Co., California; MCZC.

Distribution. United States.

Location. United States: California. 


\section{Epicauta (Epicauta) andersoni Werner, 1944}

Epicauta andersoni Werner, 1944: 66; 1945: 444; Vaurie 1950: 23; Werner et al. 1966: 40; Arnold 1976: 24; Pinto 1978: 57; 1980: 54; 1991: 273.

Terra typica. Holotype male, Gallo Springs, New Mexico; USNM.

Distribution. Mexico, United States.

Location. Mexico: Chihuahua. United States: Arizona, Colorado, Kansas, New Mexico, Oklahoma, Utah.

\section{Epicauta (Epicauta) anthracina (Erichson, 1848)}

Lytta anthracina Erichson, 1848: 566.

Epicauta anthracina: Blackwelder 1945: 482; Kaszab 1960: 41; Pinto and Bologna 2016: 205.

Terra typica. Unknown.

Distribution. Brazil, Guyana, Peru.

Location. Brazil: Amazonas. Guayana. Peru: Coronel Portillo.

New records (Figure 1): Brazil: Amazonas (Manicoré, Tefé). Peru: Coronel Portillo (Pucallpa; Río Ucayali) [MLPA].

\section{Epicauta (Epicauta) apache Pinto, 1980}

Epicauta maculata: Werner 1945: 441 (in part); 1949: 96; Dillon 1952: 393 (in part);

Werner et al. 1966: 38; Pinto 1975: 429; Arnold 1976: 23.

Epicauta apache Pinto, 1980: 62; 1991: 273.

Terra typica. Holotype male Willcox, Arizona; CASC.

Distribution. Mexico, United States.

Location. Mexico: Chihuahua, Sonora. United States: Arizona, Colorado, Kansas, Nebraska, Oklahoma, Texas.

Epicauta (Epicauta) apure Adams \& Selander, 1979

Epicauta apure Adams \& Selander, 1979: 255.

Terra typica. Holotype male, San Fernando, Apure, Venezuela; AMNH.

Distribution. Venezuela, Trinidad, Tobago.

Location. Venezuela: Apure, Monagas. Trinidad: George. Tobago: country labeled only.

Epicauta (Epicauta) aragua Adams \& Selander, 1979

Epicauta grammica: Denier 1933: 39 (in part); 1935: 20 (in part).

Epicauta aragua Adams \& Selander, 1979: 251. 
Terra typica. Holotype male, Maracay, Aragua, Venezuela; AMNH.

Distribution. Colombia, Costa Rica, El Salvador, Honduras, Panama, Venezuela.

Location. Colombia: Magdalena, Meta, Santander. Costa Rica: Guanascaste, San José Higuito. El Salvador: La Libertad, San Salvador. Honduras: Copán, Francisco Morazán. Panama: Canal Zone, Chiriquí, Coclé. Venezuela: Apure, Aragua, Bolivar, Carabobo, Distrito Federal, Guarico, Miranda, Monagas, Portuguesa, Trujillo, Zulia.

\section{Epicauta (Epicauta) assimilis (Haag-Rutembreg, 1880)}

Lytta assimilis Haag-Rutenberg, 1880: 26.

Epicauta assimilis: Denier 1935: 152; Blackwelder 1945: 482.

Terra typica. Type (s?) Rio Grande, Brazil; "Mus. Vind. and Haag-Rutenberg (in part); ZSBS.

Distribution. Argentina: Chaco, Córdoba, Misiones, Tucumán. Brazil. Paraguay: Alto Paraná. Uruguay: Cerro Largo.

New records (Figure 1): Argentina: Chaco (Charata); Córdoba (Calamuchita); Misiones (Puerto Aguirre); Tucumán (Villa Monti). Paraguay: Alto Paraná. Uruguay: Cerro Largo [MLPA, MACN].

\section{Epicauta (Epicauta) aspera Werner, 1944}

Epicauta aspera Werner, 1944: 70; 1945: 488; 1949: 105; Werner et al. 1966: 41; Arnold 1976: 24; Pinto 1991: 141.

Terra typica. Holotype male Salida, Colorado; MCZC.

Distribution. United States.

Location. United States: Arizona, Colorado, Kansas, New Mexico, Oklahoma, Texas.

\section{Epicauta (Epicauta) aterrima (Klug, 1825)}

Lytta aterrima Klug, 1825: 432; Burmeister 1881: 27.

Cantharis aterrima: Gemminger and Harold 1870: 2148; Berg 1881: 305.

Epicauta aterrima: Bruch 1911: 403; Borchmann 1917: 71; Denier 1935: 152; Blackwelder 1945: 482.

Terra typica. Type (s?) Brazil; MLPA.

Distribution. Argentina, Brazil.

Location. Argentina: Corrientes, Puerto Cazador. Brazil: Amazonas.

New records (Figure 1): Argentina: Corrientes (Puerto Cazador). Brazil: Bello Horizonte (Minas); Goyas (Río Verde); Río de Janeiro (State labeled only) [MLPA].

\section{Epicauta (Epicauta) atomaria (Germar, 1821)}

Lytta atomaria Germar, 1821: 154; Burmeister 1881: 29; Berg 1881: 307; Martínez 1992: 5. 
Cantharis germari Fischer, 1827: 24.

Cantharis atomaria: Gemminger and Harold 1870: 2148.

Epicauta atomaria: Bruch 1914: 403; Borchmann 1917: 70; Denier 1935b: 152; Bosq 1943: 10; Hayward 1942: 22; Blackwelder 1945: 482; Viana and Williner 1974: 15; Martínez 1992: 5; Di Iorio 2004: 167; Campos-Soldini 2015: 22.

Terra typica. Lytta atomaria: Type (s?) Brazil.

Distribution. Argentina, Bolivia, Brazil, Paraguay, Uruguay.

Location. Argentina: Buenos Aires, Catamarca, Córdoba, Corrientes, Entre Ríos, Formosa, Jujuy, Mendoza, Misiones, La Pampa, La Rioja, Río Negro, Santiago del Estero, Salta, San Juan, San Luis, Salta, Tucumán. Bolivia: Chiquitos, Río Negro, Santa Cruz, Santiago, Tarija. Brazil: Encruzilhada, Rio Prado, Cafapara. Paraguay: Asunción, Cordillera, Departamento de San Pedro, Guaira. Uruguay: Maldonado, Montevideo, Cerro Largo.

\section{Epicauta (Epicauta) atrata (Fabricius, 1775)}

Lytta atrata Fabricius, 1775: 260; 1781: 329; 1801: 79.

Meloe trichrus Pallas, 1798: 100.

Lytta coracina Illiger, 1804: 171 (unnecessary repl. name for atrata Fabricius, see Werner 1945: 470).

Cantharis trichrus: Fischer 1827: 23.

Lytta atrata convolvuli Melsheimer, 1846: 53.

Cantharis trichrus: Gemminger and Harold 1870: 2155.

Lytta convolvuli: Le Conte 1866: 157; Horn 1873: 97.

Epicauta convoluli: LeConte 1866: 157; Horn 1873: 97.

Cantharis convolvuli: Gemminger and Harold 1870: 2149.

Epicauta trichrus: Horn 1875: 153; Wickham 1896: 34; Blatchley 1910: 1361; Chittenden 1911: 92; Ulke 1902: 54; Sherman 1913: 246; Mutchler and Weiss 1924: 9, 18; Carruth 1931: 51

Epicauta trichura Wellman, 1910: 24 (unnecessary replacement name for trichrus Pallas; see Werner 1945: 470); Denier 1935: 159.

Epicauta pensylvanica: Borchmann 1917: 79; Staig, 1940: 135.

Epicauta atrata: Werner 1945: 470; Dillon 1952: 387; Pinto 1975: 451; 1991: 294;

Arnold 1976: 26; Selander 1981: 757; 1982: 812; Staines 1983: 46; Berríos-Ortíz 1985: 180; Lago and Mann 1987: 5; Pinto 1991: 294.

Terra typica. E. atrata Lectotype (sex?) (designated by Werner 1945) America, GUHC. E. trichrus, type locality unknown. E. convolvuli, type locality unknown.

Distribution. Canada, Mexico, United States.

Location. Canada: Manitoba. Mexico: Nuevo León, San Luis Potosí, Tamaulipas. United States: Alabama, Arkansas, District to Columbia, Florida, Georgia, Illinois, Indiana, Iowa, Kansas, Kentucky, Louisiana, Maine, Maryland, Michigan, Mississippi, Missouri, Montana, Nebraska, New Jersey, New York, North Dakota, Ohaio, Oklahoma, Pennsylvania, Sotuh Carolina, South Dakota, Texas, Virginia, West Virginia, Wyoming. 
Epicauta (Epicauta) atropos Pinto, 1991

Epicauta atropos Pinto, 1991: 299.

Terra typica. Holotype female, Mitchel, Nebraska; CASC.

Distribution. Canada, United States.

Location. Canada: Alberta. United States: Arizona, Colorado, Kansas, Montana, Nebraska, Oklahoma, South Dakota, Utah.

Epicauta (Epicauta) avellanea Denier, 1834

Epicauta avellanea Denier, 1934.

Terra typica. Holotype male, Córdoba: Valle de los Reartes. MACN.

Distribution. Argentina.

Location. Argentina: Córdoba (Valle de los Reartes).

New records (Figure 2): Argentina: Córdoba (Calamuchita, Valle de los Reartes) [MLPA].

Epicauta (Epicauta) aymara Denier, 1935

Epicauta aymara Denier, 1935: 152.

Terra typica. Holotype male, Vilaconta; MLPA. Allotype female and paratype male and female, Vilaconta; DEI.

Distribution. Peru: Apurímac.

New records (Figure 2): Peru: Apurímac (Andahuaylas) [FIMLA].

\section{Epicauta (Epicauta) basimaculata (Hagg-Rutenberg, 1880)}

Lytta basimaculata Haag-Rutenberg, 1880: 48.

Cantharis rufescens Dugès, 1881: 48.

Epicauta rufescens: Dugès 1889: 75.

Epicauta basimaculata: Champion 1892: 406; Blackwelder 1945: 482; Pinto 1982: 407; 1991: 240.

Terra typica. E. basimaculata lectotype male (designated by Pinto 1982) Mexico; ZSMC.

Distribution. Guatemala, Mexico.

Location. Guatemala: San Marcos, Talisman. Mexico: Chiapas, Colima, Guerrero, Jalisco, Mexico, Michoacán, Morelos, Nayarit, Oaxaca, Veracruz.

\section{Epicauta (Epicauta) batesii Horn, 1875}

Epicauta batesii Horn, 1875: 153; Sherman 1913: 468; Staines 1983: 46.

Epicauta batesi: Mutchler and Weiss 1924: 10, 18; Denier 1935: 153; Selander 1984:

3; Pinto 1991: 302. 


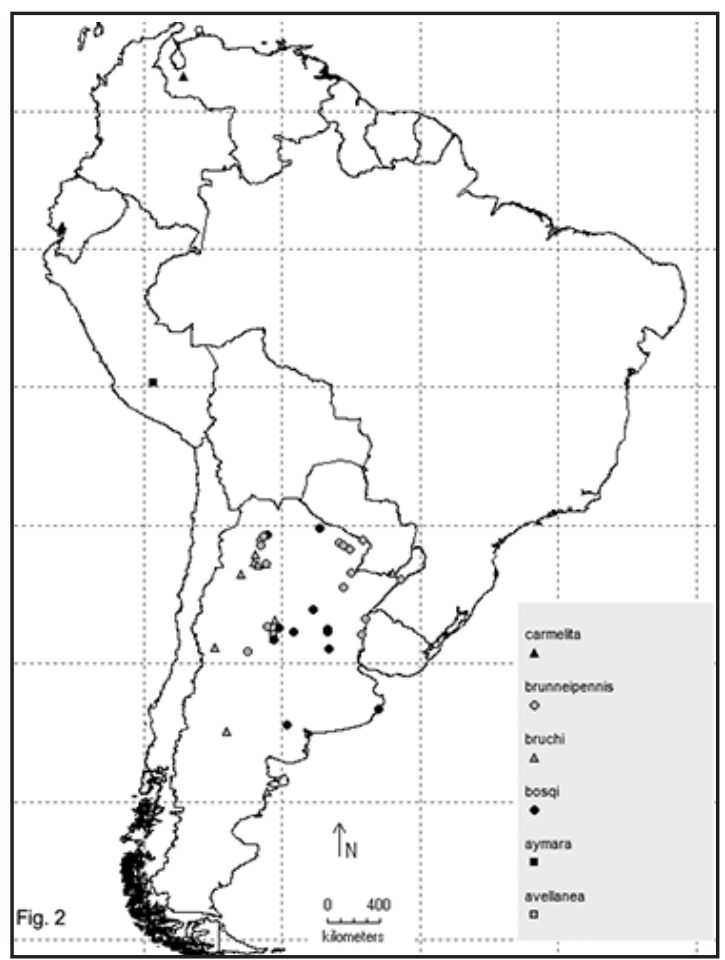

Figure 2. New distribution records for: E. avellanea; E. aymara; E. bosqi; E. bruchi; E. brunneipennis; and E. carmelita.

Terra typica. Lectotype female (designated by Werner 1945), Savannah, Georgia; MCZC.

Distribution. United States.

Location. United States: Florida, Georgia, New Jersey, Mississippi, North Carolina, South Carolina.

\section{Epicauta (Epicauta) bella Mäklin, 1875}

Epicauta bella Mäklin, 1875: 631; Borchmann 1917: 71; Denier 1935b: 153; Blackwelder 1945: 482; Campos-Soldini 2011: 578.

Lytta exclamationis Berg, 1889: 120; Bruch 1914: 405; Borchmann 1917: 94.

Terra typica. Type (s?) Lytta exclamationis, Tandil, Argentina; Uruguay; MLPA.

Distribution. Argentina, Bolivia, Uruguay.

Location. Argentina: Buenos Aires, Tandil. Uruguay (country labeled only).

\section{Epicauta (Epicauta) bispinosa Werner, 1949}

Epicauta bispinosa Werner, 1949: 95; Werner et al. 1966: 39; Pinto 1975: 435; 1980:

23, 42; 1991: 118; Arnold 1976: 27; Selander 1981: 780; Selander and Agafitei 1982: 138 . 
Terra typica. Holotype male, east of Sonoita, Arizona; MCZC.

Distribution. United States.

Location. United States: Colorado, Kansas.

Epicauta (Epicauta) boliviensi Kaszab, 1963

Epicauta boliviensis Kaszab, 1963.

Terra typica. Holotype male, Bolivia, Cochabamba; SUNM.

Distribution. Bolivia.

Location. Bolivia: Cochabamba.

Epicauta (Epicauta) borchmanni Denier, 1935

Epicauta borchmanni Denier, 1935: 153; Blackwelder 1945: 482.

Terra typica. Holotype and Allotype (sex?) Jujuy; MLPA.

Distribution. Argentina.

Location. Argentina: Jujuy.

Epicauta (Epicauta) borgmeieri Denier, 1935

Epicauta borgmeieri Denier, 1935: 153; Blackwelder 1945: 482.

Terra typica. Holotype male (?) Campos Grande, Mato Grosso, Brazil; MLPA.

Distribution. Brazil.

Location. Brazil: Mato Grosso, Campo Grande, Porto Murthino.

\section{Epicauta (Epicauta) bosqi Denier, 1935}

Epicauta bosqi Denier, 135: 135; Bosq 1943: 11; Blackwelder 1945: 482; Viana and

Williner 1974: 11; Hayward 1960: 22; Di Iorio 2004: 168; Campos-Soldini and Roig-Juñent 2011: 24.

Terra typica. Holotype female, Rosario, Santa Fe, Argenitna, MLPA.

Distribution. Argentina.

Location. Argentina: Buenos Aires, Chaco, Córdoba, Corrientes, Entre Ríos, Formosa, Misiones, Neuquén, Salta, Santa Fe, Santiago del Estero.

New records (Figure 2): Argentina: Buenos Aires (Villa Gesel); Córdoba (Baratelli, Calamuchita, Calchín); Formosa (Laguna Yema); Salta (Güemez); Santa Fe (Quebrada Bulava, La Rubia, Recreo, Rosario, Santa Fe, Santo Tomé) [IADIZA, Barriga-Tuñón, MLPA, MACN, MCNFA].

Epicauta (Epicauta) brasilera Selander, 1981

Epicauta brasilera Selander, 1981: 587.

Terra typica. Holotype male, São Domingo, Mato Grosso do Sul, Brazil; MNH. 
Distribution. Brazil.

Location. Brazil: Goiás, Mato Grosso, Mato Grosso do Sul, São Paulo.

\section{Epicauta (Epicauta) bruchi Borchmann, 1930}

Epicauta bruchi Borchmann, 1930: 93; Denier 1935b: 154; Blackwelder 1945: 482;

Martínez 1992: 5; Di Iorio 2004: 168.

Terra typica. Type (s?) Catamarca: Santa María, Tucumán: Tafí del Valle; MACN.

Distribution. Argentina.

Location. Argentina: Catamarca, Chubut, Córdoba, Mendoza, Misiones, Neuquén, Salta, Tucumán. Perú (country labeled only).

New records (Figure 2): Argentina: Catamarca (La Ciénaga, Valle de Santa María); Chubut (Province labeled only); Córdoba (Capilla del Monte); Mendoza; Misiones; Neuquén; Salta (Cafayate); Tucumán (La Quebradita, Tafí del Valle) [MLPA, FIMLA, MACN].

\section{Epicauta (Epicauta) brunnea Werner, 1944}

Epicauta brunnea Werner, 1944: 67; 1945: 454; Dillon 1952: 400; Werner et al. 1966:

33; Pinto 1982: 402; 1991: 217.

Epicauta innomina Dillon, 1952: 401.

Terra typica. E. brunnea holotype male, Alpine, Texas; MCZ.

Distribution. Mexico, United States.

Location. Mexico: Chihuaha, Sinaloa, Sonora. United States: Arizona, New Mexico, Texas.

\section{Epicauta (Epicauta) brunneipennis (Haag-Rutenberg, 1880)}

Lytta brunneipennis Haag-Rutenberg, 1880: 29.

Cantharis brunneipennis: Burmeister 1881: 24; Berg 1881: 304.

Epicauta brunneipennis: Bruch 1914: 403; Borchmann 1917: 72; Denier 1935b: 154,

1940: 419; Blackwelder 1945: 482; Viana and Williner 1974: 14; Di Iorio 2004:

168; Campos-Soldini and Roig-Juñent 2011: 579.

Terra typica. Type (s?) Buenos Aires; ZSBS.

Distribution. Argentina, Brazil, Paraguay, Uruguay.

Location. Argentina: Buenos Aires, Catamarca, Chaco, Córdoba, Corrientes, Chaco, Entre Ríos, Formosa, Mendoza, Misiones, Salta, San Luis, Tucumán. Brazil: São Paulo. Uruguay.

New records (Figure 2): Argentina: Entre Ríos (Chajarí, Parque Nacional el Palmar); Chaco (Resistencia); Córdoba (Salsacate); Corrientes (Ibarreta); Formosa (Bartolomé de las Casas, Parque Río Pilcomayo, Pirané); Misiones (Departamentode Concepción); Salta (Chicoana, La Viña, Orán, Sumalago); San Luis (San Gerónimo); Santa Fe (Villa Ana); Tucumán (province labeled only). Uruguay: country labeled only [MLPA, Barriga-Tuñón, FIMLA, MACN]. 


\section{Epicauta (Epicauta) bucephala Kaszab, 1960}

Epicauta bucephala Kaszab, 1960: 406; Pinto and Bologna 2016: 205.

Terra typica. Holotype male and Allotype female, Andahuaylas, Peru; SMB.

Distribution. Peru.

Location. Peru: Andahuaylas, 3800 m; Apurimac.

\section{Epicauta (Epicauta) callosa LeConte, 1866}

Epicauta callosa LeConte, 1866: 158; Horn 1973: 99; Packard 1889: 225; Milliken

1921: 6; Carruth 1931: 52; Denier 1935: 154; Gilberston and Horsfall 1940: 20;

Werner 1945: 468; 1949: 103; Vaurie 1950: 29; Dillon 1952: 384; Selander 1954:

24; Parker and Wakeland 1957: 26; Burke 1963: 53; Werner et al. 1966: 43; Goeden

1971; Rees 1973: 179; Arnold 1976: 25; Pinto 1977: 141; 1991: 304.

Cantharis callosa: Gemminger and Harold 1870: 2148.

Epicauta pseudocallosa Dillon, 1952: 387.

Terra typica. E. callosa, Lectotype female (designated by Werner 1945) Nebraska; MCZC. E. pseudocallosa, Holotype male, MacLennon Co., Texas; TAMU.

Distribution. Mexico, United States.

Location. Mexico: Chihuahua, Coahuila, Nuevo León, Tamaulipas. United States: Arizona, Arkansas, Colorado, Kansas, Louisiana, Montana, Nebraska, New Mexico, Oklahoma, South Dakota, Texas, Wyoming.

\section{Epicauta (Epicauta) candidata Champion, 1892}

Epicauta candidata Champion, 1892: 426; Denier 1935: 154; Blackwelder 1945: 482;

Dillon 1952: 395; Pinto 1991: 120.

Terra typica. Holotype female, Villa Lerdo, Durango; BMNH. Pinto (1991) indicates that the type is damage.

Distribution. Mexico, United States.

Location. Mexico: Chihuahua, Coahuila, Durango, Zacatecas. United States: Texas.

\section{Epicauta (Epicauta) carmelita (Haag-Rutenberg, 1880)}

Lytta carmelita Haag-Rutenberg, 1880: 46.

Epicauta carmelita: Dugès 1889: 61; Champion 1892: 417; Maydell 1934: 332; De-

nier 1935: 154; 1940: 419; Pinto 1982: 407; 1991: 123; Mathieu 1983: 158.

Terra typica. Lectotype male (designated by Pinto, 1982) "N. Granada (former name of Colombia and Panama); BMNH.

Distribution. Colombia, Costa Rica, Guatemala, Honduras, Mexico, Nicaragua, Panama, Venezuela.

Location. Costa Rica: Bebedero, Canas, La Pacifica, Santa Elena, Taboga. Guatemala: Coatepeque, Jalpatagua, San Sebastián. Honduras: Guaimaca, La Paz, Siquate- 
peque, Zamorano. Mexico: Chiapas, Oaxaca, Veracruz. Nicaragua: Chontales, Rivas. Panama: Canal Zone, El Coronero. Venezuela: Merida.

New records (Figure 2): Venezuela: Mérida (Mérida) [MLPA].

\section{Epicauta (Epicauta) cardui (Dugès, 1889)}

Henons [sic] conferta: Dugès 1869: 102.

Henons cardui Dugès, 1889: 36; Champion 1891: 368; Van Dayke 1928: 409, 411.

Epicauta cardui: Pinto 1991: 174.

Terra typica. Neotype male (designated by Pinto, 1991), Toluca, Mexico.

Distribution. Mexico.

Location. Mexico: Aguascalientes, Colima, Distrito Federal, Durango, Guanajuato, Jalisco, Mexico, Michoacán, Morelos, Oaxaca, Querétaro.

Epicauta (Epicauta) castadiva Pinto, 1991

Epicauta castadiva Pinto, 1991: 176.

Terra typica. Holotype male, south of Valle Nacional (4200’), Oaxaca, Mexico; CASC.

Distribution. Mexico.

Location. Mexico: Oaxaca.

\section{Epicauta (Epicauta) caustica Rojas, 1857}

Epicauta caustica Rojas, 1857: 441; Denier 1935: 22; Blackwelder 1945: 482; Selander 1981: 584.

Cantharis caustica: Gemminger and Harold 1870: 2148.

Lytta caustica: Haag-Rutenberg 1880: 53.

Terra typica. Neotype male (designated by Selander,1981) San Juan de las Morras, Guarico, Venezuela.

Distribution. Panamá, Venezuela.

Location. Argentina: Córdoba; Misiones. Panamá: Canal Zone. Venezuela: Apure, Aragua, Bolívar, Carabobo, Guarico, Zulia. Paraguay: Villa Florida

New records (Figure 3): Argentina: Córdoba (Calamuchita, San Miguel de los Ríos, Yacanto); Misiones (Pindapoy). Paraguay: Villa Florida [MLPA, MACN].

\section{Epicauta (Epicauta) cavernosa (Courbon, 1855)}

Cantharis cavernosa Courbon, 1855: 1006.

Cantharis cavernosa: Gemminger and Harold 1870: 2148.

Cantharis cavernosa: Berg 1881: 306.

Cantharis nigropunctata Burmeister, 1881: 28 (partim); Berg 1881: 306; Bruch 1914: 404; Borchmann 1917: 72.

Epicauta cavernosa: Borchmann 1917: 72; Bruch 1914: 404; Blackwelder 1945: 482;

Viana and Williner 1974: 15; Campos-Soldini and Roig-Juñent 2015: 24. 
Terra typica. Unknown.

Distribution. Argentina, Brazil, Uruguay.

Location. Argentina: Buenos Aires, Córdoba, Mendoza, San Luis. Brazil: country labeled only. Uruguay: Cerro Pelado, Cerro Largo, Cuchilla de Melo, Fraile Muerto, Punta del Este, Chuy.

Epicauta (Epicauta) caviceps Horn, 1873

Epicauta caviceps Horn, 1873: 99; 1874: 37; 1891: 43; Van Dyke 1928: 129; Maydell

1934: 329; Denier 1935: 154; Werner 1945: 481; Werner et al. 1966: 42; Pinto 1972: 256; 1991: 142.

Epicauta caviceps: MacSwain 1956: 59.

Terra typica. Lectotype male (designated by Werner, 1945) Arizona; MCZC.

Distribution. United States.

Location. United States: Arizona, California, Nevada, Utha.

Epicauta (Epicauta) cazieri Dillon, 1952

Epicauta cazieri Dillon, 1952: 394; Pinto 1975: 441; 1991: 119.

Terra typica. Holotype male, Sierra Blanca, Texas; AMNH.

Distribution. United States.

Location. United States: Kansas, New Mexico.

\section{Epicauta (Epicauta) cicatrix Werner, 1951}

Epicauta cicatrix Werner, 1951: 5; Pinto 1991: 144.

Terra typica. Holotype male, Presidio, Texas; USNM.

Distribution. United States.

Location. United States: Texas.

Epicauta (Epicauta) cinctipennis (Chevrolat, 1834)

Lytta cinctipennis Chevrolat, 1834: 59.

Cantharis cinctipennis: Dugès, 1869: 101; 1870: 126.

Epicauta cinctipennis: Dugès 1889: 85; Champion 1892: 420; Denier 1935: 154;

Blackwelder 1945: 483; Wirth 1956: 22; Pinto 1982: 403, 407; 1991: 164.

Terra typica. E. cinctipennis Lectotype male (designated by Pinto 1982) Mineral del Zimapan, Mexico; UZMH.

Distribution. Mexico, United States.

Location. Mexico: Guanajuato, Hidalgo, Morelos, Nuevo León, Puebla, Querétaro, San Luis Potosí. United States: Arizona, New Mexico, Texas.

Epicauta (Epicauta) cinerea (Forster, 1771)

Meloe cinerea Forster, 1771: 62. 


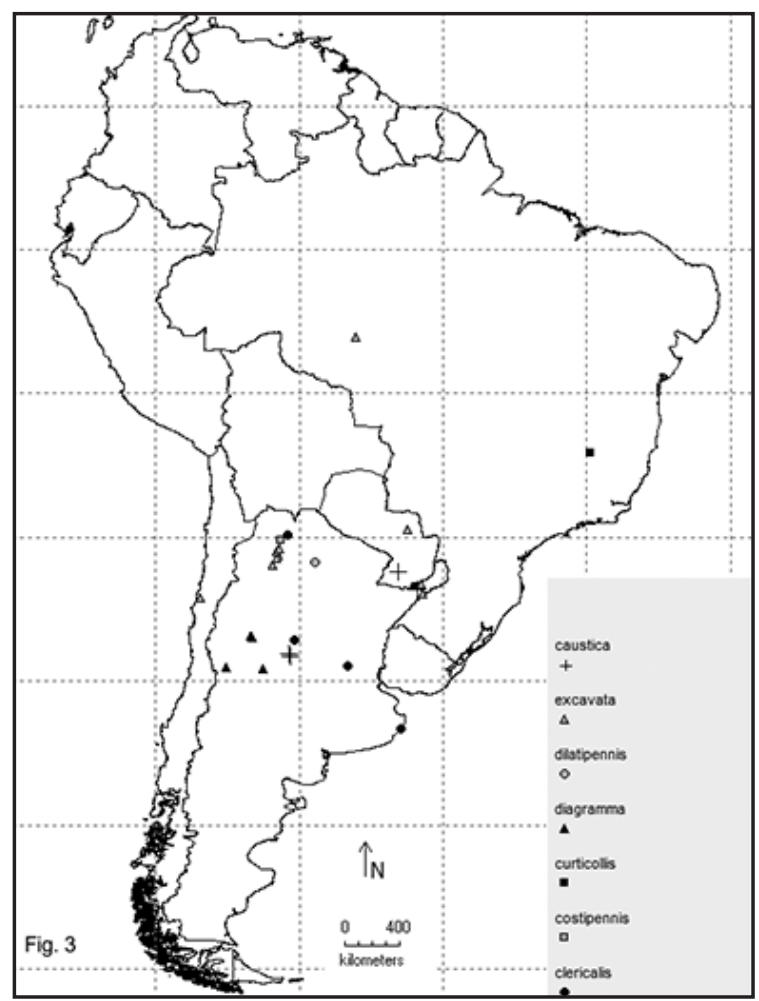

Figure 3. New distribution records for: E. clericalis; E. costipennis; E. curticollis; E. diagramma; E. dilatipennis; and E. excavata.

Meloe cinerea: Pallas 1798: 98.

Meloe clematides Woodhouse, 1800: 213.

Epicauta fissilabris LeConte, 1850: 232; Horn 1873: 102; 1885: 111; Gibson 1912:

87; Blackwelder 1945: 483; Werner 1945: 456.

Lytta cinerea: LeConte 1853: 339.

Lytta fissilabris: LeConte 1853: 339.

Cantharis cinerea: Gemminger and Harold 1870: 2148.

Epicauta cinerea: Horn 1873: 101 (in part); 1875: 153; Denier 1935: 154; Sherman

1913: 246; Carruth 1931: 300; 1970: 1786; Rees 1973: 181; Arnold 1976: 21;

Staines 1983: 47.

Epicauta (Epicauta) cinerea: MacSwain 1956: 48; Pinto 1991: 177.

Terra typica. E. cinerea, E. clematides both unknown, Pinto (1991); E. fissilabris Holotype female, Kakabeka [Falls], Lake Superior; MCZC.

Distribution. Canada, United States.

Location. Canada: Manitoba, Ontario, Saskatchewan. United States: Alabama, Arkansas, Colorado, Connecticut, Florida, Georgia, Illinois, Iowa, Kansas, Kentucky, Louisiana, Maine, Maryland, Massachusetts, Michigan, Minnesota, Mississippi, Mis- 
souri, Montana, Nebraska, New Hampshire, New Jersey, New York, North Carolina, North Dakota, Ohio, Oklahoma, Pennsylvania, Rhode Island, South Carolina, Tennessee, Texas, Virginia, West Virginia, Wisconsin.

\section{Epicauta (Epicauta) circellaris Borchmann, 1942}

Epicauta circellaris Borchmann, 1942; Pinto and Bologna 2016: 205.

Terra typica. Type (s?), Peru; type locality unknown.

Distribution. Peru.

\section{Epicauta (Epicauta) clericalis (Berg, 1881)}

Cantharis clericalis Berg, 1881: 308.

Epicauta clericalis: Borchmann 1917: 73; Bruch 1914: 404; Denier 1935: 154; Black-

welder 1945: 483; Campos-Soldini and Roig-Juñent 2011: 25.

Epicauta luteolineata var. brevebasalis Pic, 1933: 26.

Epicauta luteolineata var. discolineata Pic, 1933: 26; Denier 1935: 154; Blackwelder 1945: 483.

Terra typica. Holotype male (sex?) Misiones, Argentina, MLPA.

Distribution. Argentina.

Location. Argentina: Buenos Aires, Córdoba, Chaco, Entre Ríos, Formosa, Jujuy, Mendoza, Misiones, San Juan, Santa Fe, Santiago del Estero, Tucumán.

New records (Figure 3): Argentina: Buenos Aires (Villa Gesel); Córdoba (El Sauce); Jujuy (Ledesma); Santa Fe (Rosario) [Barriga-Tuñón, MLPA].

\section{Epicauta (Epicauta) conferta (Say, 1824)}

Meloe confertus Say, 1824: 281.

Henous techanus Haldemann, 1952: 377.

Henous confertus: LeConte 1853: 330; Riley 1877: 562; Champion 1891: 368; Van

Dyke 1928: 410; Carruth 1931: 54; Gilberston and Horsfall 1940: 19; Horsfall 1942: 93; 1943: 20.

Henous texanus: LeConte 1883: 167; Vaurie 1950: 25; Selander and Pinto 1967: 411;

Selander and Weddler 1969: 32; Rees 1973: 183; Arnold 1976: 20; Agafitei and

Selander 1980: 11; Selander 1981: 777; Selander and Agafitei 1982: 141; Berríos-

Ortíz 1985: 180. Pinto1991: 210.

Epicauta (Henous) conferta: MacSwain 1956: 45.

Terra typica. E. conferta Neotype female (designated by Werner 1945) Dallas, Texas; MCZC.

Distribution. Mexico, United States.

Location. Mexico: Chihuaha, Nuevo León. United States: Arkansas, Illinois, Iowa, Kansas, Missouri, Nebraska, New Mexico, Oklahoma, South Dakota, Texas. 
Epicauta (Epicauta) convergenta Kaszab, 1963

Epicauta convergenta Kaszab, 1963: 338; Pinto and Bologna 2016: 205.

Terra typica. Holotype male, Machupichu, Cuzco, Peru; SUNM.

Distribution. Peru.

Records. Peru: Cusco.

Epicauta (Epicauta) cora García-París \& Ruiz, 2013

Epicauta cora García-París \& Ruiz, 2013: 59.

Terra typica. Holotype sex?) Nayarit, Huaynamota, Los Sabinos, Mexico; CNINIBUNAM.

Distribution. Mexico.

Lacality records. Mexico: Nayarit, Río Huaynamota, Los Sabinos.

Epicauta (Epicauta) corvina (LeConte, 1858)

Lytta corvina LeConte, 1858: 21.

Cantharis corvina: Gemminger and Harold 1870: 2149.

Epicauta corvina: Horn 1873: 96, 102; 1885: 111; Dugès 1877: 66; Champion 1892:

418; Milliken 1921: 6; Cockerell and Harris 1925: 32; Carruth 1931: 52; Denier 1935: 154; Blackwelder 1945: 483; Werner 1945: 446; Vaurie 1950: 23; Dillon 1952: 389; Werner et al. 1966: 32; Pinto 1973: 968; 1977: 139; 1991: 220; Arnold 1976: 19; Pinto and Mayor 1986: 602.

Terra typica. Lectotype female (designated by Werner 1945) Valley of the Gila, Arizona; MCZC.

Distribution. Mexico, United States.

Location. Mexico: Chihuahua, Coahuila, Durango, Sinaloa, Sonora. United States: Arizona, Colorado, Iowa, Nebraska, New Mexico, Oklahoma, South Dakota, Texas, Wyoming.

Epicauta (Epicauta) corybantica Pinto, 1991

Epicauta corybantica Pinto, 1991: 145.

Epicauta alphonsii: Fall 1901: 184; Werner 1945: 480; 1955: 3; Pinto 1972: 256.

Epicauta alphonsei: Moore 1937: 42.

Terra typica. Holotye male, S. Adelanto, San Bernardino Co., California.

Distribution. United States.

Location. United States: California.

Epicauta (Epicauta) costata (LeConte, 1854)

Lytta costata LeConte, 1854: 84; 1858: 23. 
Pleuropompha costata: LeConte 1862: 273; LeConte and Horn 1883: 421; Cockerell and Harris 1925: 30; Werner 1943: 31; 1945: 426; Vaurie 1950: 38; Dillon 1952: 378; Gupta 1965: 457; 1971: 14; Werner et al. 1966: 53; Goeden 1971: 47, 48; Pinto 1973: 957; 1977: 135; Arnold 1976: 30; Selander 1981: 780.

Cantharis costata: Gemminger and Harold 1870: 2149.

Epicauta costata: Pinto 1984: 381; 1991: 228; Pinto and Mayor 1986: 602.

Terra typica. Holotype female Frontera (Rio Grande), New Mexico: MCZC.

Distribution. Mexico, United States.

Location. Mexico: Chihuahua, Coahuila, Durango, Sonora. United States: Arizona, California, New Mexico, Oklahoma, Texas.

\section{Epicauta (Epicauta) costipennis Borchmann, 1930}

Epicauta costipennis Borchmann, 1930: 93; Denier 1935: 154; Blackwelder 1945: 483;

Campos-Soldini et al. 2009: 4.

Terra typica. Type (s?) Troquero, Jujuy; MACN.

Distribution. Argentina.

Location. Argentina: Jujuy.

New records (Figure 3): Argentina: Jujuy (Torquero) [MLPA].

\section{Epicauta (Epicauta) crassitarsis Maydell, 1935}

Epicauta crassitarsis Maydell, 1935: 72; Denier 1940: 419; Werner 1945: 439; Werner et al. 1966: 38; Adams and Selander 1979: 234; Pinto 1991: 241.

Terra typica. Holotype male, Tempe, Arizona; USNM.

Distribution. Mexico, United States.

Location. Mexico: Sinaloa, Sonora. United States: Arizona.

\section{Epicauta (Epicauta) crucerea Selander, 1981}

Epicauta crucerea Selander, 1981: 589.

Terra typica. Holtype male, Chiquitos, Santa Cruz, Bolivia; MNH.

Distribution. Bolivia.

Location. Bolivia: Santa Cruz (San José de Chiquitos, 700 m).

\section{Epicauta (Epicauta) cupraeola (Dugès, 1869)}

Cantharis cupraeola Dugès, 1869: 112; Horn 1885: 107; Vásquez and Zaragoza 1979: 578.

Cantharis rufipedes Dugès, 1870: 163.

Cantharis cinctella Dugès, 1877: 59.

Lytta subvittata Haag-Rutenberg, 1880: 47; Pinto, 1982: 47.

Epicauta insignis Horn, 1885: 110; Werner 1945: 437; Dillon 1952: 397; Werner et al.

1966: 37; Pinto 1977: 137. 
Epicauta rufipedes: Dugès 1889: 64; Champion 1892: 407; Denier 1935: 22; Vaurie 1950: 17; Selander 1954: 87; 1959: 210; Pinto 1982: 404.

Epicauta cinctella: Dugès 1889: 69.

Epicauta vittula Beauregard, 1889: 113 (n repl. name for E. subvittata (Haag-Rutenberg) nec Erichson, 1848).

Epicauta cupraeola: Champion 1982: 408; Dugès 1889: 69; Denier 1935: 152; 1940: 419; Blackwelder 1945: 483; Pinto 1991: 244; García-París and Ruiz 2013.

Terra typica. E. cupraeola Holotype male, "en los cerros de Guanajauto" lost. Neotype male (designated by Pinto 1991); BMNH. E. rufipedes Types (s) Hacienda de la Noria, Michoacán, Mexico; CASC. E. cinctella Type (s) states of Veracruz, Mexico, lost; Neotype male (designated by Pinto, 1991); CASC. E. subvittata Lectotype male (designated by Pinto 1982) Mexico; UZMH. E. insignis Lectotype male (designated by Werner, 1945) Arizona; MCZC.

Distribution. Costa Rica, El Salvador, Guatemala, Honduras, Mexico, Nicaragua, United States.

Location. Costa Rica: Alajuela, Bebedero, Cabo Santa Elena, La Pacifica, Las Canas, Leberia, Palo Verde, Turrialba, Turrubales. El Salvador: La Unión, Quezaltepeque, San Andrés, San Salvador. Guatemala: Guatemala, Managua, Rabinal, Sacapulas, Salama, San Gerónimo, Zacapa. Honduras: Siguatepeque, Tegucigalpa. Mexico: Aguascalientes, Campeche, Chiapas, Chihuahua, Cohauila, Colima, Distrito Federal, Durango, Guanajuato, Guerrero, Jalisco, Mexico, Michoacán, Morelos, Nayarit, Quintana, San Luis de Potosí, Sinaloa, Sonora, Veracruz, Yucatán, Zacatecas. Nicaragua: Chontales, Jinotega, Managua, Nandaime, Rivas. United States: Arizona, New Mexico.

\section{Epicauta (Epicauta) curticollis Borchmann, 1930}

Epicauta curticollis Borchmann, 1930: 92.

Terra typica. Type (s?) male and female, Santa Trinidad, Paraguay; type locality unknown.

Distribution. Argentina: Misiones. Brazil: Mina Gerais. Paraguay (country labeled only).

New records (Figure 3): Argentina: Misiones (Province labeled only). Brazil: Mina Gerais (Diamantina) [MLPA].

\section{Epicauta (Epicauta) curvicornis (Haag-Rutenberg, 1880)}

Lytta curvicornis Haag-Rutenberg, 1880: 54.

Lytta funebris Haag-Rutenberg, 1880: 44.

Macrobasis antennalis Dugès 1881: 148; 1889: 54.

Epicauta curvicornis: Champion 1892: 406; Dinier 1935: 154; Blackwelder 1945: 483

Pinto 1982: 407; 1984: 378; 1991: 264.

Epicauta (Epicauta) curvicornis: Werner 1973: 461.

Terra typica. E. curvicornis Lectotype male (designated by Pinto 1982) Mirador, Veracruz, Mexico; BMNH. 
Distribution. Mexico.

Location. Mexico: Guerrero, Michoacán, Morelos, Oaxaca, Puebla, Veracruz.

Epicauta (Epicauta) delicata Mathieu, 1983

Epiauta delicata Mathieu, 1983: 158; Pinto 1984: 381; 1991: 230.

Terra typica. Holotype male, Paila, Coahuila.

Distribution. Mexico.

Location. Mexico: Coahuila.

Epicauta (Epicauta) diagramma (Burmeister, 1881)

Cantharis diagramma Burmeister, 1881: 24.

Cantharis griseonigra: Berg 1881: 304 (partim).

Lytta griseonigra: Borchmann 1917: 405 (partim).

Epicauta diagramma: Denier 1935b: 154; 1940: 419; Blackwelder 1945: 483; Campos-Soldini 2011: 580.

Terra typica. Type (s?) Uruguay; type locality unknown.

Distribution. Argentina, Uruguay.

Location. Argentina: Buenos Aires: Tandil; Mendoza; San Juan; San Luis. Uruguay (country labeled only).

New records (Figure 3): Argentina: Mendoza (Cerro Cacheuta); San Juan (Las Tumanas, Valle Fértil); San Luis (San Gerónimo) [IADIZA, Barriga-Tuñón, FIMLA, MACN].

Epicauta (Epicauta) diana Pinto, 1991

Epicauta diana Pinto, 1991: 253. García-París and Ruiz 2013.

Terra typica. Holotype male, Villa Unión, Sinaloa, Mexico; CASA.

Distribution. Mexico.

Location. Mexico: Oaxaca, Sinaloa.

Epicauta (Epicauta) dilaticornis Borchmann, 1952

Epicauta dilaticornis Borchmann, 1952; Pinto and Bologna 2016: 205.

Terra typica. Holotype female, Peru, Pampa de Cangallo; type locality unknown.

Distribution. Peru.

Location. Peru: Ayacucho; Pampa de Cangallo, 3450 m; Querobamba; Tayapampa $4025 \mathrm{~m}$.

Epicauta (Epicauta) dilatipennis Pic, 1916.

Epicauta dilatipennis Pic, 1916: 8; Denier 1935b: 154, 1940: 419; Blackwelder 1945:

483; Campos-Soldini and Roig-Juñent 2015: 26. 
Terra typica. Unknown.

Distribution. Argentina.

Location. Argentina: Misiones, Santiago del Estero.

New records (Figure 3): Argentina: Santiago del Estero (San Ignacio) [MACN].

\section{Epicauta (Epicauta) diversipubescens Maydell, 1934}

Epicauta diversipubescens Maydell, 1934: 333; Denier 1940: 420; Werner 1945: 487;

1949: 106, 108; 1954: 110; 1955: 5; Pinto 1991: 147.

Terra typica. Holotype male, Albuquerque, New Mexico; USNM.

Distribution. United States.

Location. United States: New Mexico, Texas.

\section{Epicauta (Epicauta) emarginata Champion, 1892}

Epicauta emarginata Champion, 1892: 426; Vaurie 1950: 30; Denier 1935: 155;

Blackwelder 1945: 483; Werner 1954: 105; Werner et al. 1966: 44; Pinto 1982: 407; 1991: 307.

Epicauta calcarata Werner, 1944: 70; 1945: 477; Dillon 1952: 416.

Terra typica. E. emarginata, Lectotype male (designated by Pinto 1982) San Isidro, Coahuila, Mexico; BMNH.

Distribution. Mexico, United States.

Location. Mexico: Chihuahua, Coahuila. United States: Arizona, New Mexico, Texas.

Epicauta (Epicauta) ennsi Werner, 1957

Epicauta ennsi Werner, 1957: 97; Hoebeke 1978: 4; Pinto 1991: 308.

Terra typica. Holotype male, north of Rockport, Aransas Co., Texas; SEMC.

Distribution. United States.

Location. United States: Texas.

\section{Epicauta (Excavata) excavata (Klug, 1825)}

Cantharis excavata Klug, 1825: 440; Gemminger and Harold 1870: 2150

Cantharis sulcifrons Chevrolat, 1829: 135; Gemminger and Harold 1870: 2154

Epicauta excavata: Borchmann 1917: 74; Denier 1935: 155, 1940: 420; Blackwelder

1945: 483. Martinez 1992: 5-6; Di lorio 2004: 168; Campos-Soldini and RoigJuñent 2011: 26.

Terra typica. Unknown.

Distribution. Argentina, Brazil, Paraguay.

Location. Argentina: Misiones, Jujuy, Salta. Brazil: Río Grande do Soul. Chile: Huasco. Paraguay: San Pedro. 
New records (Figure 3): Argentina: Misiones (Departamento de Concepción, Departamento de Santa María, San Ignacio); Salta (El Abisal, Coronel Moldes, Corralito, La Viña, Rosario de Lerma, Sumalago). Brazil: Matto Grosso (Rancho Grande). Chile: Huasco (Canto del Agua). Paraguay: San Pedro (Cororo) [Barriga-Tuñón, MACN].

\section{Epicauta (Epicauta) excavatifrons Maydell, 1934}

Epicauta excavatifrons Maydell, 1934: 330; Denier 1940: 420; Werner 1945: 484; 1955: 5; Selander 1984: 3; Pinto 1991: 149.

Terra typica. Holotype female, Ocala, Marion Co., Florida; ANSP.

Distribution. United States.

Location. United States: Alabama, Florida, Mississippi.

\section{Epicauta (Epicauta) falcolarandina García-París et. al., 2016}

Epicauta falcolarandina García-París, Ruiz, Sánchez-Vialas \& López-Estrada, 2016: 946.

Terra typica. Holotype female, Venezuela, Parapara; CNIN-IBUNAM.

Distribution. Venezuela.

Location. Venezuela: Falcon, Lara.

\section{Epicauta (Epicauta) fallax Horn, 1885}

Epicauta fallax Horn, 1885: 111; Fall 1901: 184; Denier 1935: 155; Werner 1945:

450; 1954: 108; Ballmer 1980: 83; Pinto 1991: 332.

Epicauta ensiformis Werner, 1944: 68.

Terra typica. E. fallax, Lectotype male (designated by Pinto 1991) Owens Valley, California; MCZC.

Distribution. United States.

Location. United States: California.

\section{Epicauta (Epicauta) ferruginea (Say, 1824)}

Lytta ferruginea Say, 1824: 298; LeConte 1853: 341.

Cantharis ferruginea: Gemminger and Harold 1870: 2150.

Epicauta ferruginea: LeConte 1866; Horn 1873: 153; 1875: 153; 1891: 43; Champion 1892: 425; Wickham 1896: 34; Milliken 1921: 6; Carruth 1931: 51; Denier 1935: 155; Werner 1945: 465; Vaurie 1950: 28; Dillon 1952: 386; Parker and Wakeland 1957: 26; Gupta 1965: 454; Hatch 1965: 107; Werner et al. 1966: 43; Church 1967: 756; Rees 1973: 187; Arnold 1976: 25; Kumar et al. 1976: 23; Lavigne 1976: 755; Pinto 1991: 309.

Terra typica. Neotype female (designated by Werner 1945) Cambridge, Nebraska; MCZC.

Distribution. Canada, Mexico, United States. 
Location. Tipical Race: Canada: Alberta, Manitoba, Saskatchewan. Mexico: Chihuahua. United States: Arizona, Colorado, Idaho, Kansas, Missouri, Montana, Nebraska, Nevada, New Mexico, North Dakota, Oklahoma, South Dakota, Texas, Utah, Wyoming. East Race: United State: Texas.

\section{Epicauta (Epicauta) flavogrisea (Burmeisteir, 1881)}

Lytta flavogrisea Haag-Rutenberg, 1880: ?.

Cantharis flavogrisea Burmeister, 1881: 29.

Epicauta flavogrisea: Denier 1935: 155; Blackwelder 1945: 483;

Terra typica. Type (s?) Buenos Aires: Bahía Blanca; presumably in ZSBS.

Distribution. Argentina: Buenos Aires. Paraguay.

New records (Figure 4): Argentina: Buenos Aires (Sierra de la Ventana) [MACN].

Epicauta (Epicauta) flobcina Pinto, 1991

Epicauta flobcina Pinto, 1991: 182.

Terra typica. Holotype male, Easton, Pennsylvania; CASC.

Distribution. United States.

Location. United States: Alabama, District of Columbia, Georgia, Illinois, Iowa, Kentucky, Massachusetts, Maryland, Minnesota, Nebraska, New Hampshire, New Jersey, New York, North Carolina, Pennsylvania, Virginia, West Virginia, Wisconsin.

Epicauta (Epicauta) floridensis Werner, 1944

Epicauta floridensis Werner, 1944: 68; 1945: 458; Arnold 1976: 22; Staines 1983: 47;

Selander 1984: 3; Pinto 1991: 184.

Epicauta pseudosolani: Dillon 1952: 395 (in part).

Terra typica. Holotype male, Sebring, Florida; MCZC.

Distribution. United States.

Location. United States: Alabama, Florida, Illinois, Louisiana, Missippi, Missouri, New Jersey, Oklahoma, South Carolina, Texas.

\section{Epicauta (Epicauta) floydwerneri Martínez, 1955}

Lytta rubriceps Blanchard, 1843: 200.

Epicauta floydwerneri Martínez, 1955: 58; 1992: 6. (New name for E. rubriceps (Blanchard, 1843), not E. rubriceps (Redtenbacher, 1842)).

Terra typica. Type (s?) Santa Cruz, Bolivia; MNHN, location unknown.

Distribution. Argentina, Bolivia, Brazil, Paraguay.

Location. Argentina: La Rioja, Mendoza, Jujuy, Misiones, Salta. Bolivia: Santa Cruz de la Sierra, El Cidral, Estación de Experimentación Agrícola Saavedra. Brazil: Parabía. Paraguay: Colonia Nueva Italia, Departamento de Amambay. 
New records (Figure 4): Argentina: Misiones (Concepción); Salta (Alemanía, Capiazuti, Chiloana, Coronel Moldes, La Viña, Pocitos, Rosario de Lerma, San Martín, Sumalago). Paraguay: Departamento de Amambay [Barriga-Tuñon, MACN].

\section{Epicauta (Epicauta) fortis Werner, 1944}

Epicauta fortis Werner, 1944: 69; 1945: 466; Vaurie 1950: 28; Dillon 1952: 386; Werner et al. 1966: 43; Pinto 1975: 451; Arnold 1976: 25; Pinto 1991: 25.

Terra typica. Holotype male, Phoenix, Arizona; MCZC.

Distribution. Mexico, United States.

Location. Typical Race: Mexico: Baja California Norte, Baja California Sur, Chihuahua, Sonora. United States: Arizona, California, Kansas, Nevada, New Mexico, Oklahoma, Texas. East Texas Race: Mexico: Tamaulipas. United States: Texas.

\section{Epicauta (Epicauta) franciscana Denier, 1935}

Epicauta franciscana Denier, 1935: 155; Blackwelder 1945: 483; Campos-Soldini et al. 2009: 4.

Terra typica. Holotype male, Rosario, Santa Fe, Argentina; MLPA.

Distribution. Argentina, Uruguay.

Location. Argentina: Buenos Aires, Santa Fe, La Rioja. Uruguay: Cerro Largo (Departament labeled).

New records (Figure 4): Argentina: Santa Fe (Rosario) [MLPA].

\section{Epicauta (Epicauta) fuliginosa (Oliver, 1795)}

Cantharis fuliginosa Oliver, 1795: 14.

Epicauta fuliginosa: Borchmann 1917: 75.

Terra typica. Type (s?) unspecified locality; location, possibly in the MNHN.

Distribution. Colombia: country labeled only.

\section{Epicauta (Epcauta) fulvicornis (Burmeister, 1881)}

Cantharis fulvicornis Burmeister, 1881: 29; Berg, 1881: 307.

Epicauta fulvicornis: Bruch 1914: 404; Denier 1935: 155; Bosq 1943: 11; Hayward 1942: 22; Blackwelder 1945: 483; Martínez 1992: 6; Di Iorio 2004: 168; Campos-Soldini and Roig-Juñent 2011: 26.

Epicauta testaceicornis Pic, 1916: 8.

Lytta fulvicornis: Borchmann 1917: 94.

Epicauta fourcadei Denier, 1939: 179; Blackwelder 1945: 483; Campos-Soldini et al. 2009: 4.

Terra typica. two Types Cantharis fulvicornis, Mendoza, province labeled only; MACN. Holotype (sex?) Epicauta fourcadei, Argentina; MLPA. $1-3)$ is deposited at MLPA (La Plata, Argentina). 


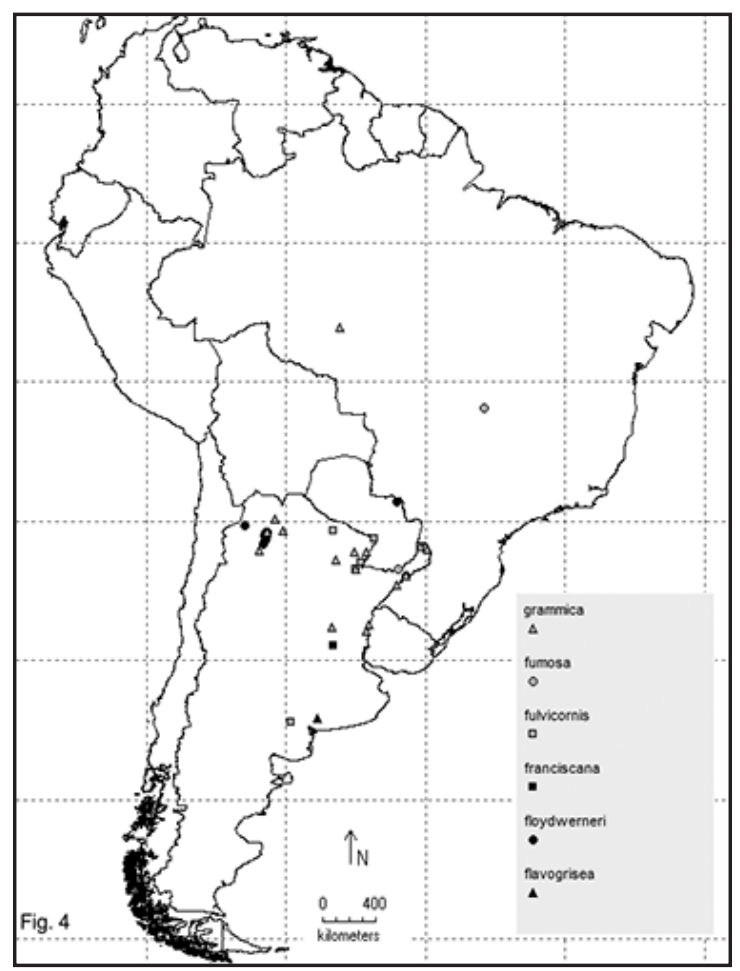

Figure 4. New distribution records for: E. flavogrisea; E. floydwerneri; E. franciscana; E. fulvicornis; E. fumosa; and E. grammica.

Distribution. Argentina, Paraguay.

Location. Argentina: Chaco, Corrientes, Entre Ríos, Fromosa, La Rioja, Misiones, Santa Fe, Santiago del Estero, Salta, Tucumán.

New records (Figure 4): Argentina: Chaco (General Vedia, Río Tapengo); Formosa (Clorinda, Las Lomitas); Misioines (Chulumani, Iguazú) [MLPA, FIMLA, MACN].

\section{Epicauta (Epicauta) fumosa (Germar, 1824)}

Lytta fumosa Germar, 1824: 173.

Cantharis fumosa: Gemminger and Harold 1870: 2150.

Anomalonyx fumosus Denier, 1935: 161.

Anomalonychus fumosus Sailor, 1940: 46; Kaszab 1963: 340.

Epicauta fumosa: Borchmann 1917: 75; Blackwelder 1945: 483; Pinto 1984: 378.

Terra typica. Anomalonychus fumosus Holotype male, Alotype female and one Paratype, Santa Catharina, Brazil in the Sammlung des Museums G. Frey. Anomalonyx fumosus; MLPA location unknown.

Distribution. Argentina, Brazil.

Location. Argentina: Misiones. Brazil: Goyaz.

New records (Figure 4): Argentina: Misiones (Province labeled only). Brazil: Goyaz [MACN]. 


\section{Epicauta (Epicauta) funebris Horn, 1873}

Epicauta funebris Horn, 1873: 102; Milliken 1921: 6; Denier 1935: 23; Horsfall 1943:

41; Werner 1945: 447; Dillon 1952: 389; Selander and Weddle 1969: 37; Rees 1973: 188; Arnold 1976: 20; Selander 1981: 777; Pinto 1977: 141; 1991: 186.

Epicauta cinerea: Riley 1877: 551, 561; Chittenden 1903: 24; Milliken 1921: 6; Rees 1973: 181

Epicauta pestifera Werner, 1949: 100 (n, repl. name for E. solani Werner, 1945 nec

Denier, 1940); Gupta 1965: 455; Selander and Weddler 1969: 37; Arnold 1976:

21; Adams and Selander 1979: 173, 217; Selander 1981: 777; 1982: 427; 1984:

3; Staines 1983: 48; Lago and Mann 1987: 5; MacCormik and Carrel 1987: 310;

Blodgett and Higging 1988: 1461

Epicauta (Epicauta) solani: MacSwain 1956: 46.

Terra typica. E. funebris Lectotype (designated by Werner 1945) Texas; MCZC. E. pestifera Holotype male, Norwood Pennsylvania; MCZC.

Distribution. United States.

Location. United States: Alabama, Arkansas, Connecticut, Delaware, District of Columbia, Florida, Georgia, Illinois, Indiana, Louisiana, Maryland, Massachusetts, Michigan, Minnesota, Mississippi, Missouri, Nebraska, New Hampshire, New Jersey, New York, North Carolina, Ohio, Oklahoma, Pennsylvania, Rhode Island, South Carolina, South Dakota, Tennessee, Texas, Virginia, West Virginia, Wisconsin.

\section{Epicauta (Epicauta) geniculata (Haag-Rutenberg, 1880)}

Lytta geniculata Haag-Rutenberg, 1880: 28.

Epicauta geniculata: Blackwelder 1945: 483.

Terra typica. Type (?s) Brazil, presumably in ZSBS.

Distribution. Argentina, Brazil, countries labeled only.

\section{Epicauta (Epicauta) grammica (Fischer, 1827)}

Cantharis grammica Fischer, 1827: 19; Gemminger and Harold 1870: 2151.

Epicauta grammica: Borchmann 1917: 75; Denier 1935: 155, 1940: 420; Blackwelder

1945: 483; Martínez 1992: 6; Campos-Soldini and Roig-Juñent 2011: 27.

Epicauta fidelis Brethes, 1925: 14.

Terra typica. Cantharis grammica, Type (s?) Brazil; RMM, present location unknown. E. fidelis Holotype (?) Santa Fe, Argentina; MACN.

Distribution. Argentina, Bolivia, Brazil, Colombia, Costa Rica, El Salvador, Guatemala, Honduras, Panama, Paraguay, Uruguay and Venezuela.

Location. Argentina: Chaco, Córdoba, Corrientes, Entre Ríos, Formosa, Jujuy, Misiones, Salta, Santa Fe, Rio Negro, Santa Fe, Salta, Tucumán. Paraguay: Departamento de San Pedro. Bolivia: Cochabamba. Brazil: Mato Grosso, Minas Gerais, São Paulo. Colombia: Magdalena, Meta, Santander, Vélez, Guanacaste, Puntarenas, San José, Coyolar. El Salvador: La Libertad. Guatemala: Izabal, Cuyotenango. Honduras: 
Copán, Francisco Morazón. Panama: Canal Zone, Coclé, Panamá. Uruguay: Rio Negro. Venezuela: Apure, Aragua, Bolívar, Carabobo, Distrito Federal, Miranda, Monagas, Portuguesa, Sucre, Trujillo, Zulia.

New records (Figure 4): Argentina: Chaco (Resistencia, Saenz Peña); Corrientes (Santo Tomé); Entre Ríos (Concordia, Parque Nacional el Palmar); Formosa (Campos Villafañe, Río Pilcomayo); Jujuy (Ledesma); Misiones (Iguazú, Piñalito, Santa María); Salta (Cafayate, Las Lajitas, Paso del Rey, Sumalao); Santa Fe (Piquete). Brazil: Mato Grosso (Rancho Grande) [Barriga-Tuñon, MLPA, MACN].

\section{Epicauta (Epicauta) griseonigra (Fairmaire, 1873)}

Cantharis griseonigra Fairmaire, 1873: 73; Berg 1881: 304 (partim).

Cantharis centralis Burmeister, 1881: 25.

Lytta griseonigra: Bruch 1914: 405 (partim).

Epicauta centralis var. ochraceocincta Pic, 1916: 22

Epicauta griseonigra: Borchmann 1917: 76 (partim); Denier 1935b: 156 (partim);

Blackwelder 1945: 483 (partim); Viana and Williner 1974: 15; Martínez 1992:

6-7; Campos-Soldini 2011: 581.

Epicauta centralis: Borchmann 1917: 72; Bruch 1914: 404; Blackwelder 1945: 483;

Di Iorio 2004: 168.

Terra typica. Cantharis centralis, Type (s?) Córdoba, La Rioja, Santiago del Estero; MHNP.

Distribution. Argentina, Uruguay.

Location. Argentina: Catamarca, Córdoba, Entre Ríos, Formosa, La Rioja, Mendoza, Salta, San Juan, San Luis, Santiago del Estero, Tucumán. Uruguay: country labeled only.

New records (Figure 5): Argentina: Córdoba (Calamuchita, El Sauce, Mansilla); Formosa (Bartolomé de las Casas, El Colorado, Laguna Yema); Salta (Chioacán, Salta Forestal); San Juan (Las Tunas); San Luis (El Balde, San Gerónimo) [Barriga Tunón].

\section{Epicauta (Epicauta) heterodera Horn, 1891}

Epicauta heterodera Horn, 1891: 43; Maydell 1934: 333; Denier 1935: 156; Werner 1945: 478; Selander 1984: 3; Pinto 1991: 315.

Epicauta watsoni Blatchley, 1918: 58.

Terra typica. E. heterodera Lectotype female (designated by Werner 1945) northern Florida; MCZC. E. watsoni, Holotype female, near Gainsville, Florida; PURC.

Distribution. United States.

Location. United States: Alabama, Florida, Georgia, Louisiana, Mississippi, North Carolina.

\section{Epicauta (Epicauta) hieroglyphica (Haag-Rutenberg, 1880)}

Lytta hieroglyphica Haag-Rutenberg, 1880: 26.

Epicauta hieroglyphica: Borchmann 1917: 76; Blackwelder 1945: 483. 
Terra typica. Type (s?) Brazil; presumably in ZSBS.

Distribution. Colombia and Brazil, countries labeled only.

New records (Figure 5): Colombia: Barranquilla (Distrit labeled only) [MLPA].

\section{Epicauta (Epicauta) horni Champion, 1892}

Cantharis cinerea Dugès, 1869: 160.

Cantharis vicina Dugès, 1881: 147 (inappropriate repl. name). Horn 1885: 107.

Epicauta vicina: Dugès 1889: 70.

Epicauta horni: Champion 1892: 412 (n. repl. name of E. cinereas (Dugès), nec Foster,

1771; and for E. vicina (Dugès), nec Haag-Rutenberg, 1880); Denier 1935: 156;

Blackwelder 1945: 483; Pinto 1991: 274.

Terra typica. Neotype male (designated by Pinto 1991) Guanajuato, Mexico; BMNH.

Distribution. Mexico.

Location. Mexico: Colima, Guanajuato, Tamaulipas.

\section{Epicauta (Epicauta) hubbelli Werner, 1973}

Epicauta (Epicauta) hubbelli Werner, 1973: 460.

Epicauta hubbelli: Pinto 1984: 378; 1991: 266.

Terra typica. Holotype male, Mexico, Chaipas; MCZC.

Distribution. Mexico.

Location. Mexico: Chiapas; Oaxaca.

\section{Epicauta (Epicauta) imitatrix Kaszab, 1960}

Epicauta imitatrix Kaszab, 1960: 405; Pinto and Bologna 2016: 205.

Terra typica. Holotype male and Allotype female, Puna de Andahuaylas, 4000 m, Peru; SMB.

Distribution. Peru.

Location. Peru: Apurímac, Ayacucho, Huanta, 2660 m.

Epicauta (Epicauta) impressifrons Van Dayke, 1928

Epicauta impressifrons Van Dayke, 1928: 262; 1929: 129; Denier 1935: 156; Werner 1945: 482; 1945: 482; 1949: 103, 105; 1955: 5; Pinto 1972: 256; 1991: 150.

Terra typica. Holotype male, Plam Springs, Riverside Co., California; CASC.

Distribution. United States.

Location. United States: California.

\section{Epicauta (Epicauta) inconstants (Fischer, 1827)}

Cantharis inconstants Fischer, 1827: 17; Gemminger and Harold 1870: 2151.

Epicauta inconstants: Borchmann 1917: 76; Denier 1935: 156; 1940: 420; Blackwelder 1945: 483. 


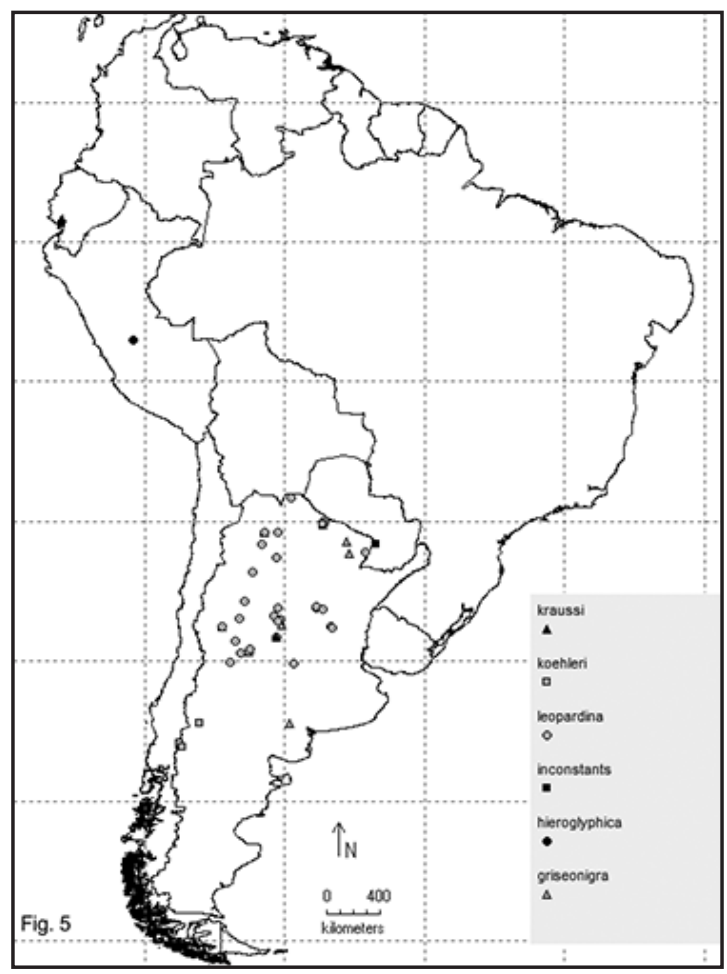

Figure 5. New distribution records for: E. griseonigra; E. hieroglyphica; E. inconstants; E. leopardina; E. koebleri; and E. kraussi.

Terra typica. Unknown.

Distribution. Paraguay, Brazil.

Location. Paraguay: Villeta. Brazil (Country labeled only).

New records (Figure 5): Paraguay: Villeta (Colonia Nueva Italia) [MLPA].

\section{Epicauta (Epicauta) insueta Werner, 1955}

Epicauta insueta Werner, 1955: 11; Pinto 1991: 151.

Terra typica. Holotype male, Toluca, Mexico; AMNH.

Distribution. Mexico.

Location. Mexico: Toluaca.

\section{Epicauta (Epicauta) jeffersi Pinto, 1980}

Epicauta normalis: Werner, 1949: 96 (in part); Werner et al. 1966: 38 (in part); Arnold 1976: 23.

Epicauta jeffersi Pinto, 1980: 56; 1991: 275.

Terra typica. Holotype male, southeast of Willcox, Arizona; CASC.

Distribution. United States.

Location. United States: Arizona, Colorado, Oklahoma. 


\section{Epicauta (Epicauta) jimenezi Dugès, 1889}

Epicauta jimenezi Dugès, 1889: 73; Champion 1892: 417; Denier 1935: 156; Vaurie

1950: 23; Blackwelder 1945: 483; Werner 1954: 110; Werner et al. 1966: 32;

Vázquez and Zaragoza 1979: 580; Pinto 1991: 222.

Epicauta nigropilosa Maydell, 1934: 332; Denier 1940: 421.

Terra typica. E. jimenezi Holotype (sex?) Guadalajara, Mexico; UNAM. E. nigropilosa Holotype (sex?) Guadalajara, Mexico; UNAM.

Distribution. Mexico, United States.

Location. Mexico: Durango, Jalisco, Sinaloa, Sonora. United States: Arizona.

\section{Epicauta (Epicauta) kansanas Werner, 1944}

Epicauta kansanas Werner, 1944: 70; 1945: 476; Ballamer 1980: 84; Pinto 1991: 332.

Terra typica. Holotype male, type locale unknown, erroneously labeled "Sedgewick Co., Kansas"; USNM.

Distribution. United States.

Location. United States: Kansas.

\section{Epicauta (Epicauta) koebleri Denier, 1940}

Epicauta koehleri Denier, 1940: 420; Bosq 1943: 11; Viana and Williner 1974: 11; Di

Iorio 2004: 168; Campos-Soldini and Roig-Juñent 2015: 28.

Terra typica. Holotye male, Allotype female, Santa Fe: Sancti Spiritu; MLPA, location unknown.

Distribution. Argentina, Bolivia.

Location. Argentina: Buenos Aires, Chubut Mendoza, Golfo San Jorge, Neuquén, San Juan, Santa Cruz, Santa Fe, Río Negro. Bolivia: Nor Yungas, La Paz.

New records (Figure 5): Argentina: Formosa (Bermejo, Laguna Yema); Neuquén (Lago Verde, San Martín de los Andes, Río Agrio) [IADIZA, FIMLA, MACN].

Epicauta (Epicauta) koehleri var. solani Denier, 1940

Epicauta koehleri var. solani Denier, 1940: 421.

Terra typica. Holotype and Allotype (sex?), Argentina; MLPA, location unknown.

Distribution. Argentina.

Location. Argentina: Mendoza, Neuquén, San Juan, Santa Cruz.

Epicauta (Epicauta) korytkowskii Kaszab, 1978

Epicauta korytkowskii Kaszab, 1978: 331; Pinto and Bologna 2016: 205.

Terra typica. Type (s?) Santa Cruz, Peru; type locality unknown.

Distribution. Peru. 
Location. Peru: Cajamarca; Santa Cruz.

Epicauta (Epicauta) kraatzi (Haag-Rutenberg, 1880)

Lytta kraatzi Haag-Rutenberg, 1880: 22.

Epicauta kraatzi: Borchmann 1917: 76.

Terra typica. Type (s?) Brazil; presumably in ZSBS.

Distribution. Brazil (Country labeled only).

Epicauta (Epicauta) kraussi (Haag-Rutenberg, 1880)

Lytta kraussi Haag-Rutenberg, 1880: 25.

Epicauta kraussi: Borchmann 1917: 97.

Terra typica. Type (s?) Irisanga, Brazil, in the collection of the "Mus. Vind. and HaagRutenberg (in part); ZSBS.

Distribution. Argentina: Córdoba. Brazil: Goyaz (Río Verde).

New Records (Figure 5): Argentina: Córdoba (Calamuchita) [MLPA].

Epicauta (Epicauta) laevicornis Werner, 1973

Epicauta (Epicauta) laevicornis Werner, 1973: 458.

Epicauta laevicornis: Pinto 1984: 378; 1991: 267.

Terra typica. Holotyopes male, Mountains and canyons north of Ajijie, Jalisco, Mexico; MCZC.

Distribution. Mexico.

Location. Mexico: Colima, Guerrero, Jalisco, Mexico, Michoacán.

Epicauta (Epicauta) latitarsis (Haag-Rutenberg, 1880)

Lytta latitarsis Haag-Rutenberg, 1880: 33.

Epicauta latitarsis: Blackwelder 1945: 483; Pinto and Selander 2016: 205.

Terra typica. Type (s?) Peru, Coll: Haag-Rutenberg; presumably in ZSBS.

Distribution. Peru.

Location. Peru: Ayacucho.

Epicauta (Epicauta) leopardina (Hagg-Rutenberg, 1880)

Lytta leopardina Haag-Rutenberg, 1880: 30.

Cantharis leopardina: Burmeister 1881: 24; Berg, 1881: 304.

Epicauta leopardina: Bruch 1914: 404; Borchmann 1917: 77; Denier 1935: 156; Bosq

1934: 327; 1942: 11; Blackewelder 1945: 483; Viana and Williner 1974: 11; Martinez 1992: 7; Di Iorio 2004: 169; Campos-Soldini and Roig-Juñent 2015: 27. 
Terra typica. Syntipes (sex?) Córdoba, Argentina; in the Haag-Rutenberg Collection, ZSBM.

Distribution. Argentina, Brazil, Colombia.

Location. Argentina: Buenos Aires, Catamarca, Córdoba, Chaco, Entre Ríos, Formosa, Mendoza, Misiones, Neuquén, Salta, San Juan, San Luis, Santa Fe, Santiago del Estreo, Tucumán. Brazil: Goyaz, Río de Janeiro. Colombia: Atlántico.

New records (Figure 5): Argentina: Catamarca (Andalgalá); Córdoba (Agua de Oro, Calamuchita, Cruz del Eje, Ischilín Quilino, La Falda, Laboulage, La Falda); La Rioja (Talamuyuna); Formosa (Laguna Yema, Palmar Largo); Mendoza (Desaguadero, Lavalle, Nacuñan); Salta (Alemanía, Carapari, Figueroa, María Juana, Piquete); San Juan (Las Rumanas, Valle Féritl); San Luis (Balde, San Gerónimo); Santa Fe (Arrufo, Colastiné Sur, La Rubia, Recreo, Rincón Norte, San Cristobal); Tucumán (Gobernador Garmendia) [IADIZA, FIMLA, MACN, MLPA, MCNFA].

\section{Epicauta (Epicauta) leucocoma Champion, 1892}

Epicauta leucocoma Champion, 1892: 425; Denier 1935: 156; Pinto 1982: 407; 1991 :

254.

Terra typica. Lectotype male (designated by Pinto 1982) Tepanistlahuaca (= Tepanixtlahuaca) Oaxaca, Mexico.

Distribution. Mexico.

Location. Mexico: Guerrero, Jalisco, Oaxaca.

\section{Epicauta (Epicauta) limbaticollis Pic, 1924}

Epicauta limbaticollis Pic, 1924: 32; Pinto and Bologna 2016: 205.

Terra typica. Type (s?) Peru; type locality unknown.

Distribution. Peru: country labeled only.

\section{Epicauta (Epicauta) lizeri Denier, 1934}

Epicauta lizeri Denier, 1934: 271; Denier 1940: 421; Blackwelder 1945: 483; Campos-Soldini et al. 2009: 5; Campos-Soldini and Roig Juñent 2015: 29.

Terra typica. Holotype, allotype, and eight paratypes (sex?), Argentina: MLPA; two paratypes, location unknown; MACN.

Distribution. Argentina, Bolivia.

Location. Argentina: Catamarca, Chaco, Jujuy, La Rioja, Salta, Santa Cruz, Santiago del Estero, Tucumán. Bolivia: Santa Cruz.

New records (Figure 6): Argentina: Salta (province labeled only); Tucumán (Tafí del Valle). Bolivia: Santa Cruz (Lagunilla); [MLPA, FIMLA, MACN, MLPA].

\section{Epicauta (Epicauta) luctifera (Fairmaire, 1873)}

Cantharis luctifera Fairmaire, 1873: 534; Berg 1881: 303. 
Cantharis leucoloma Burmeister, 1881: 22.

Lytta luctifera: Bruch 1914: 405.

Epicauta luctifera: Borchmann 1917: 77; Bosq 1943: 11; Hayward 1942: 23; Blackwelder 1945: 483; Viana and Williner 1974: 14; Di Iorio 2004: 169; CamposSoldini 2011: 582 .

Terra typica. Cantharis leucocoma, Type (s?) Uruguay; type locality unknown.

Distribution. Argentina, Uruguay.

Location. Argentina: Buenos Aires; Córdoba, San Luís, Tucumán. Uruguay (Montevideo).

New records (Figure 6): Argentina: Córdoba (Calamuchita, El Sauce). Uruguay: Florida (Arroyo Chico); Montevideo: (Minas) [Barriga-Tunón, MLPA].

\section{Epicauta (Epicauta) luteolineata Pic, 1933}

Epicauta luteolineata Pic, 1933: 25.

Epicauta missionum var luteolineata: Denier 1935: 157; 1940: 421; Blackwelder 1945:

483; Di Iorio 2004: 169; Campos-Soldini and Roig-Juñent 2011: 38.

Terra typica. Syntypes (sex?), "Río Salado" Argentina; type locality unknown.

Distribution. Argentina.

Location. Argentina: Mendoza, Misiones, Santa Fe; Salta, Santiago del Estero, Tucumán.

\section{Epicauta (Epicauta) maculata (Say, 1823)}

Lytta maculata Say, 1823: 298; LeConte 1853: 340.

Lytta conspersa LeConte, 1853: 340; 1866: 158.

Cantharis maculata: Gemminger and Harold 1870: 2151.

Epicauta maculata: LeConte 1866: 158; Denier 1935;156; Gilberston and Horsfall 1940: 14; Larson 1943: 480; Werner 1944: 65; 1945: 441; 1949: 95 (in part);

Vauri 1950: 19; Dillon 1952: 393; (in part); Arnold 1976: 23; Pinto 1980: 64;

1991: 275; Bouseman 1986: 366.

Cantharis punctata: Dugès 1870: 161.

Epicauta media Dugès, 1889: 82.

Epicauta conspersa: Champion 1892: 413.

Epicauta nogales Werner, 1944: 65; 1945: 442; 1949: 96; Dillon 1952: 391; Werner et al. 1966: 39; Pinto 1975: 430.

Terra typica. E. maculata: Neotype male (designated by Werner 1945) Indianola, Nebraska; MCZC. E. conspersa Lectotype male (designated by Werner 1945) Missoury. E. punctata apparently lost. E. media Holotype male, Nogales, Arizona; MCZC.

Distribution. Canada, Guatemala, Mexico, United States.

Location. Canada: Manitoba, Saskatchewan. Guatemala: Chichicastenango, Huehuetenango. Mexico: Aguascalientes, Chihuahua, Coahuila, Distrito Federal, Du- 
rango, Guanajuato, Hidalgo, Jalisco, Michoacan, Nuevo León, Oaxaca, Queréteros, Sonora, Zacatecas. United States: Arizona, Colorado, Illinois, Iowa, Kansas, Montana, Nebraska, New Mexico, North Dakota, Oklahoma, South Dakota, Texas.

\section{Epicauta (Epicauta) magnomaculata Martin, 1932}

Epicauta magnomaculata Martin, 1932: 169; Maehler 1939: 65; Denier 1940: 421;

Werner 1945: 445; Pinto 1980: 46; 1991: 276.

Terra typica. Holotype male, Ballart, Inyo Co., California; CASC.

Distribution. United States.

Location. United States: California.

Epicauta (Epicauta) major Pic, 1924

Cantharis marginata: Dugès 1877: 59.

Epicauta marginata: Dugès 1889: 78.

Epicauta cinerea: Champion 1892: 421.

Epicauta cinctipennis major Pic, 1924: 2.

Epicauta subatra: Denier 1935: 22.

Epicauta major: Pinto 1991: 191.

Terra typica. Syntypes from southern Tamaulipas and San Luis Potosí south; MNHP.

Distribution. Costa Rica, Guatemala, Honduras, Mexico, Panama.

Location. Costa Rica: Carillo, Cartago, Dos Ríos, Hamburg Farm, Juan Viñas, La Estrella, San Carlos. Guatemala: Senahu, Panzos, Tikal. Honduras: Tela. Mexico: Campeche, Chiapas, Oaxaca, Quintana Roo, San Luis Potosí, Tabasco, Veracruz, Yucatan. Panama: Cerro Salud, Maje Island.

\section{Epicauta (Epicauta) mexicana (Dugès, 1889)}

Henous mexicanus Dugès, 1889: 37; Champion, 1892: 369; Van Dyke 1928: 409. Epicauta mexicana Pinto, 1991: 196.

Terra typica. Lectotype male (designated by Pinto 1991) Mexico; HMNH.

Distribution. Mexico.

Location. Mexico: Hidalgo, Veracruz.

\section{Epicauta (Epicauta) minutepunctata Borchmann, 1930}

Epicauta minutepunctata Borchmann, 1930: 94; Denier 1935: 157; Blackwelder 1945: 483; Campos-Soldini and Roig-Juñent 2015: 31.

Epicauta rosilloi Martínez, 1952: 255; Campos-Soldini and Roig-Juñent 2015.

Terra typica. Type (s?) Mendoza: Pedregal; MACN.

Distribution. Argentina.

Location. Argentina: Buenos Aires, Jujuy, Salta, San Luis: San Francisco, Tucumán. 


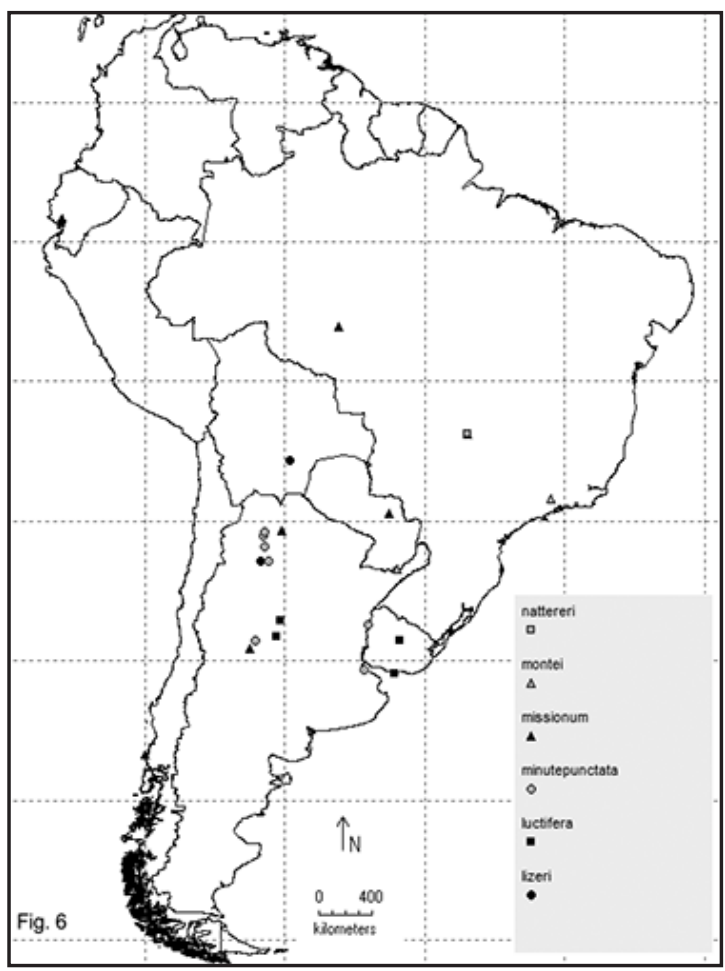

Figure 6. New distribution records for: E. lizeri; E. luctifera; E. minutepunctata; E. missionum; E. montei; and E. nattereri.

New records (Figure 6): Argentina: Buenos Aires (province labeled only); Entre Ríos (Concordia); Salta (Chicoana, Salta Forestal, Tabillar) San Luis (San Framcisco); Tucumán (Río Mediano) [MLPA, Barriga-Tunón, FIMLA, MACN].

\section{Epicauta (Epicauta) mirabilis Kaszab, 1963}

Epicauta mirabilis Kaszab, 1963: 338; Pinto and Bologna 2016: 205.

Terra typica. Holotype male, Ayacucho, Peru; SUNM.

Distribution. Peru.

Location. Peru: Ayacucho.

\section{Epicauta (Epicauta) missionum (Berg, 1881)}

Cantharis missionum Berg, 1881: 306.

Cantharis clericalis Berg, 1881: 308

Epicauta missionum Borchmann, 1971: 78; Denier 1935b: 157; Blackwelder 1945:

483; Campos-Soldini et al. 2009: 5; Campos-Soldini and Roig-Juñent 2015: 38.

Epicauta luteolineata var. brevebasalis Pic, 1933: 26.

Epicauta luteolineata var. discolineata Pic, 1933: 26; Denier 1935: 154; Blackwelder, 1945: 483. 
Epicauta missionum var. luteolineata: Denier 1935b: 157; 1940: 421; Blackwelder 1945: 483; Di lorio 2004: 169.

Epicauta clericalis var. discolineata: Denier 1935: 154; Blackwelder 1945: 483.

Terra typica. Holotype female, Misiones, Argentina; MLPA.

Distribution. Argentina, Brazil, Uruguay.

Location. Argentina: Buenos Aires, Córdoba, Entre Ríos, Formosa, Jujuy, La Rioja, Mendoza, Misiones, Neuquén, Rosario, Salta, San Juan, San Luis, Santa Fe, Santiago del Estero, San Luis, Tucumán. Brazil: Matto Grosso. Paraguay: San Pedro de Cororo. Uruguay: Rivera.

New records (Figure 6): Argentina: San Luis (Las Lajitas, San Gerónimo); Misiones (Lagunilla). Brazil: Matto Grosso (Rancho Grande). Paraguay: San Pedro (Cororo). Uruguay: Rivera (Sierra de la Rivera) [Barriga-Tuñon].

\section{Epicauta (Epicauta) mixta Dugès, 1889}

Lytta neglecta Haag-Rutenberg, 1880: 54.

Epicauta mixta Dugès, 1889: 83; Vázquez and Zaragoza 1979: 580; Pinto 1982: 404;

1991: 224; Pinto and Mayor 1986: 602.

Epicauta neglecta: Champion 1982 (in major part).

Terra typica. Holotype (?) Oaxaca, Mexico; UNAM.

Distribution. Mexico.

Location. Mexico: Chiapas, Distrito Federal, Guerrero, Hidalgo, Mexico, Michoacán, Morelos, Oaxaca, Veracruz.

\section{Epicauta (Epicauta) modesta (Haag-Rutenberg, 1880)}

Lytta modesta Haag-Rutenberg, 1880: 53.

Epicauta modesta: Champion 1892: 423; Denier 1935: 157; Blackwelder 1945: 483;

Pinto 1991: 125.

Terra typica. Holotype male, Mexico; ZSMC.

Distribution. Mexico, country labeled only.

\section{Epicauta (Epicauta) monachica (Berg, 1883)}

Lytta monachica Berg, 1883: 68.

Epicauta monachica: Blanchard 1891: 495; Bruch 1914: 404; Borchmann 1917: 78;

Denier 1935b: 157; Bosq 1934: 327; 1943: 11; Hayward 1942: 23; Blackwelder 1945: 483; Viana and Williner 1974: 87; Martinez 1992: 7; Di Iorio 2004: 170; Campos-Soldini et al. 2009: 5; Campos-Soldini and Roig-Juñent 2011: 39.

Terra typica. Lectotype female (designated by Adams and Selander 1979) Rodeo del Medio, Mendoza, Argentina; MLPA.

Distribution. Argentina, Bolivia, Brazil. 
Location. Argentina: Catamarca; Córdoba, Chaco, La Rioja: Chilecito, Quebrada de Olta, Santiago del Estero, Salta, Santa Fe, Catamarca, Córdoba, Corrientes, Chaco, Formosa, La Rioja, Mendoza, Misiones, Salta, San Juan, Santa Fe, Santiago del Estero, Tucumán. Bolivia: Lagunilla. Brazil: Mato Grosso.

Epicauta (Epicauta) monrosi Kaszab, 1960

Epicauta monrosi Kaszab, 1960: 402; Pinto and Bologna 2016: 205.

Terra typica. Holotype and Paratype male, Cuzco, 3200-4200 m, Peru; SUMB.

Distribution. Peru.

Location. Peru: Cusco.

Epicauta (Epicauta) montara Ballmer, 1980

Epicauta montara Ballmer, 1980: 85; Pinto 1991: 333.

Terra typica. Holotype male, Montara, San Mateo Co., California; CASC.

Distribution. United States.

Location. United States: California.

\section{Epicauta (Epicauta) montei Denier, 1935}

Epicauta montei Denier, 1935: 157; Blackwelder 1945: 483; Dilorio 2004: 170; Campos-Soldini et al. 2009: 5 .

Terra typica. Holotype (sex?), Allotype (sex?) and Paratype (s?) Brazil: Mina Gerais, Bello Horizonte; Goyaz, Río Verde; Río de Janeiro, Mendes; Río Grande do Soul. Uruguay: Cerro Largo; in the Sección de Entomología del Instituto de Biología Vegetal de Río de Janeiro.

Distribution. Brazil, Uruguay.

Location. Argentina: Misiones. Brazil: Bello Horizonte (Mina Gerais); Goyaz: Rio Verde; Rio de Janeiro: Mendes; Rio Grande do Soul. Uruguay: Cerro Largo.

New records (Figure 6): Argentina: Misiones. Brazil: Goyaz: Rio Verde; Mina Gerais (Passa quatro) [MLPA].

Epicauta (Epicauta) nattereri Haag-Rutenberg, 1880

Epicauta nattereri Haag-Rutenberg, 1880: 78; Denier 1935: 157; Blackwelder 1945: 483.

Terra typica. Type (s?) Irizanga, Brazil; "Mus. Vind. and Haag-Rutenberg; ZSBS.

Distribution. Brazil.

Location. Brazil: Goyaz.

New records (Figure 6): Brazil: Goyaz (Río Verde).

Epicauta (Epicauta) nigrans Mäklin, 1875

Epicauta nigrans Mäklin, 1875; Pinto and Bologna 2016: 205. 
Terra typica. Type (s?) Peru; type locality unknown.

Distribution. Peru (country labeled only).

\section{Epicauta (Epicauta) nigropunctata (Blanchard, 1843)}

Cantharis nigropunctata Blanchard, 1843: 200; Gemminger and Harold 1870: 2152.

Cantharis nigropunctata: Burmeister 1881: 28 (partim).

Epicauta nigropunctata: Borchmann 1917: 79; Denier 1935b: 158; Blackwelder 1945:

483; Bosq 1942: 11; Di Iorio 2004: 170; Campos-Soldini and Roig-Juñent 2015:

32.

Epicauta breyeri Denier, 1934: 273; 1935b: 154; Blackwelder 1945: 482.

Terra typica. E. breyeri Holotype male, Allotype female, La Rioja: Patquia; MACN; MLPA location unknown.

Distribution. Argentina, Bolivia, Brazil, Uruguay.

Location. Argentina: Catamarca, Chaco, Córdoba, Corrientes, Misiones, Mendoza, Salta. Bolivia Chulumaní, Coroico. Brazil: Parana. Uruguay: Montevideo.

New records (Figure 7): Argentina: Catamarca (Cuesta del Totoral); Chaco (El Zapallar); Córdoba (Calamuchita, La Serranita); Corrientes (Santo Tomé); Mendoza (Nihuil); Misiones (Apostoles, Iguazú, Pindapoy, San Ingacio, San Javier, Santa María); Salta (Cabra Corral, Corralito, El Alisal, José de Metan). Brazil: Parana (Guaraniacú) [MLPA, Barriga-Tuñón, MACN].

Epicauta (Epicauta) nigerrima (Dugès, 1870)

Cantharis nigerrima Dugès, 1870: 161.

Epicauta nigerrima: Dugès 1889: 77; Pinto 1991: 225.

Epicauta corvina: Horn 1885: 107; Champion 1892: 418; Werner 1945: 446.

Terra typica. Pinto (1991) indicates that three males in the Salle Collection (BMNH) are from León.

Distribution. Mexico.

Location. Mexico: Aguascaliente, Durango, Guanajuato, Jalisco, Michoacán, Nuevo León, Querétaro, Sinaloa.

\section{Epicauta (Epicauta) nigritarsis (LeConte, 1853)}

Lytta nigritarsis LeConte, 1853: 340.

Cantharis nigritarsis: Gemminger and Harold 1870: 2152.

Epicauta nigritarsis: Horn 1873: 100; 1885: 111; Denier 1935: 158; Werner 1945:

438; 1949: 105; Dillon 1952: 396; Selander 1959: 211; Werner et al. 1966: 37;

Arnold 1976: 23; Pinto 1977: 137; 1982: 403; 1991: 282; Adams and Selander

1979: 234; Pinto and Mayor 1986: 602.

Epicauta hesitata Dilon, 1952: 398.

Epicauta (Epicauta) nigritarisis Dillon, 1952: 398. 
Terra typica. E. nigritarsis Lectotype female (designated by Werner 1945) Mexican Boundary (Texas); MCZC. E. hesitata Holotype male, Belton, Texas; TAMU.

Distribution. Mexico, United States.

Location. Mexico: Coahulia, Nuevo León, Tamaulipas. United States: Arizona, New Mexico, Oklahoma, Texas.

\section{Epicauta (Epicauta) normalis Werner, 1944}

Epicauta normalis Werner, 1944: 65 (in part); 1949: 95 (in part); Vaurie 1950: 2 (in part); Dillon 1952: 392; Hatch 1965: 105; Werner et al. 1966: 38 (in part); Lavigne and Pfadt 1966: 7; Church 1967: 756; Kumar et al. 1967: 23; Rodgers and Lavigne 1972: 19; Pinto 1975: 430; 1980: 58; 1991: 277.

Terra typica. Holotype male, Bridgeport, California; MCZC.

Distribution. Canada, United States.

Location. Canada: Alberta, Saskatchewan. United States: Arizona, California, Colorado, Idaho, Kansas, Montana, Nevada, New Mwxico, North Dakota, South Dakota, Texas.

\section{Epicauta (Epicauta) obesa (Chevrolat, 1835)}

Lytta obesa Chevrolat, 1835: 81.

Cantharis obesa: Dugès 1870: 128; Gemminger and Harold 1870: 2152.

Lytta mus Haag-Rutenberg, 1880: 55.

Epicauta obesa: Dugès 1889: 66; Champion 1892: 424; Denier 1935: 158; Blackwelder 1945: 483; Werner 1949: 100; Pinto 1982: 403; 1991: 196.

Epicauta auricomans Champion, 1982: 424; Vaurie 1950: 28; Pinto 1982: 403. Epicauta ficta Werner, 1949: 100; Arnold 1976: 21; Hoebeke 1978: 14. Epicauta pseudosolani Dillon, 1952: 395.

Terra typica. E. obesa two syntypes (?) Toulepeck (=Toulepec), Mexico; UZMH.

Distribution. Canada, Mexico, United States.

Location. Canada: Ontario. Mexico: Distrito Federal, Hidalgo, Jalisco, Michoacán, Morelos, Nuevo León, Puebla, Querétaro, San Luis Potosí, Tamaulipas, Veracruz. United States: Arkansas, Connecticut, Florida, Georgia, Illinois, Iowa, Massachusetts, Michigan, New Hampshire, New Mexico, North Carolina, New York, Ohio, Oklahoma, Pennsylvania, South Carolina, Texas, Wisconsin.

\section{Epicauta (Epicauta) occidentalis Werner, 1944}

Epicauta lemniscata: Horn 1873: 100; Ortenburger and Hatch 1926: 144; Ingram 1927: 1; Ingram and Douglas 1932: 71; Horsfall 1943: 32 (in part); Douglas 1935: 686; Werner 1945: 463; Dillon 1952: 389.

Epicauta vittata: Bruner 1891: 15; Cockerell and Harris 1925: 31; Tafenelli and Bess 1968: 51. 


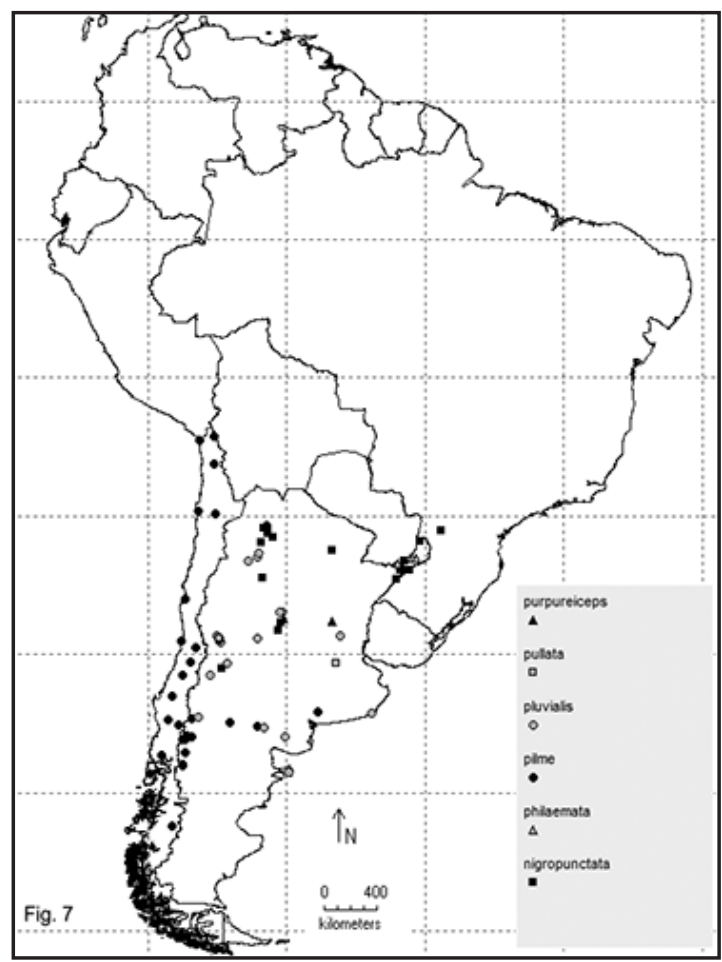

Figure 7. New distribution records for: E. nigropunctata; E. philaemata; E. pilme; E. pluvialis; E. pullata; and E. purpureiceps.

Epicauta occidentalis Werner, 1944: 69; 1945: 465; Dillon 1952: 399; Adams and Selander 1979: 246; Blodgett and Higgins 1988: 1456; Ray et al. 1989: 187; Pinto 1991: 348.

Epicauta (Epicauta) lemniscata: MackSwain 1956: 60.

Terra typica. Holotype male, Cambridge, Nebraska; MCZC.

Distribution. United States.

Location. United States: Alanbama, Arkansas, Colorado, Georgia, Illinois, Indiana, Kansas, Kentucky, Louisiana, Mississippi, Missouri, Nebraska, Oklahoma, South Dakota, Tennessee, Texas.

\section{Epicauta (Epicauta) ocellata (Dugès, 1870)}

Cantharis ocellata Dugès, 1870: 160; Horn 1885: 107.

Epicauta ocellata: Champion 1892: 414; Denier 1935: 158; Blackwelder 1945: 483;

Selander 1959: 211; Pinto 1980: 70; 1991: 278.

Terra typica. Neotype male (designated by Pinto, 1980) Atlixco, Mexico; CASC.

Distribution. Mexico. 
Location. Mexico: Coahuila, Durango, Morelos, Oaxaca, San Luis Potosí.

Epicauta (Epicauta) ochropus (Haag-Rutenberg, 1880)

Lytta ochropus Haag-Rutenberg, 1880: 28.

Epicauta ochropus: Blackwelder 1945: 483.

Terra typica. Type (s?) Brazil, presumably in ZSBS.

Distribution. Brazil, contry labeled only.

\section{Epicauta (Epicauta) oregona Horn, 1875}

Epicauta oregona Horn, 1875: 153; Denier 1935: 158; Werner 1945: 461; Parker and

Wakeland 1957: 26; 1975: 432; 1965: 105; Werner et al. 1966: 34; Church 1967:

755; Rees 1973: 199; Pinto 1975: 432, 435; 1980: 42; 1991: 286.

Terra typica. Lectotype male (designated by Werner 1945) Oregon; MCZC.

Distribution. Canada, Unted States.

Location. Canada: Alberta, British Columbia, Manitoba, Saskatchewan. Unted States: Arizona, Colorado, Idaho, Montana, Nebraska, Nevada, New Mexico, Dakota, Utah, Washington.

\section{Epicauta (Epicauta) pardalis LeConte, 1866}

Epicauta pardalis LeConte, 1866: 157 (as race of E. maculata); Horn 1873: 96 (as species); Cahmpion 1892: 414; Denier 1935: 158; Blackwelder 1945: 484; Werner 1944: 65; 1945: 443; Vaurie 1950: 21; Dillon 1952: 392; Selander 1954: 87;

Werner et al. 1966: 40; Pinto 1975: 43; 1980: 50; Ray et al. 1989: 187.

Epicauta (Epicauta) pardalis: MacSwain 1956: 58; Pinto 1991: 278.

Terra typica. Lectotype male (designated by Werner 1945) Valley of the Gila; MCZC.

Distribution. Mexico, United States.

Location. Mexico: Aguascalientes, Chihuahua, Coahuila, Durango, Sonora. United States: Arizona, New Mexico, Texas.

\section{Epicauta (Epicauta) parvula (Haldeman, 1853)}

Meloe parvus Haldeman, 1852: 377; LeConte 1853: 329.

Meloe parvulus Haldeman, 1853: 404 (n. repl. name for M. parvus Haldeman, nec Solier, 1851).

Namaspis parvulus: Van Dyke 1928: 411.

Epicauta parva: Werner 1945: 449; Kumar et al. 1976: 23; Lavigne 1976: 754; Agafitei and Selander 1980: 13.

Epicauta parvula: Pinto 1991: 212.

Terra typica. Type (s) Santa Fe (Pinto 1991) inthicates that the type (s) species is unknown.

Distribution. United States. 
Location. United States: Colorado, Kansas, Nebraska, New Mexico.

\section{Epicauta (Epicauta) pedalis LeConte, 1866}

Epicauta pedalis LeConte, 1866: 157; Horn 1873: 99; 1885: 111; 1894: 356; Denier

1935: 158; Werner 1945: 440; 1949: 93-95; Pinto 1991: 255.

Cantahris pedalis: Gemminger and Harold 1870: 2152.

Terra typica. Lectotype male (designated by Werner 1945; erroneously as female) Cape San Lucas, Baja Calisfornia Sur, Mexico.

Distribution. Mexico.

Location. Mexico: Baja California Sur.

\section{Epicauta (Epicauta) pensylvanica (Degeer, 1775)}

Cantharis pensylvanica Degeer, 1775: 15.

Meloe nigra Woodhouse, 1800: 213.

Lytta pensylvanica: LeConte 1853: 339; 1854: 447.

Lytta morio LeConte, 1854: 447.

Cantharis pennsylvanica: Gemminger and Harold 1870: 2152.

Epicauta pensylvanica: Riley 1877: 561; Champion 1892: 418; Wickham 1896: 34;

Ulke 1902: 54; Chittenden 1903: 25; 1911: 92; Blatchely 1910: 1363; Gibson 1912: 83; Sherman 1913: 246; Rau and Rau 1916: 260; Milliken 1921: 6; Mutchler and Weiss 1924: 10, 18; Cocerell and Harris 1925: 31; Gowdey 1926: 13; Carruth 1931: 52; Staig 1940: 135; Horsfall 1914: 114; 1943: 13; Denier 1935: 158; Blackwelder 1945: 484; Vaurie 1950: 25; Dillon 1952: 388; Selander 1954: 24; 1981: 757; 1982: 156, 427; 1984: 2; Selander and Bouseman 1960: 202; Parker and Wakeland 1957: 26; Gupta 1965: 454; Werner et al. 1966: 33; Church 1967: 754; Mathwig 1968: 544; Selander and Weddle 1969: 36; Rees 1973: 200; Arnold 1976: 20; Kumar et al. 1976: 23; Lavigne 1976: 754; Adams and Selander 1979: 160, 172; Werner et al. 1980: 1405; McLain 1982: 396; Staines 1983: 47; Capinera et al. 1985: 1054; Goldburg 1987: 247; Lago and Mann 1987: 5; Blodgett and Higgins 1988: 1456; Ray et al. 1989: 187.

Epicauta pensylvanica: Horn 1873: 102; Werner 1945: 447.

Lytta atrata: Claypole 1881: 31.

Epicauta potosina Dugès, 1889: 89.

Epicauta (Epicauta) pensylvanica: MacSwain 1956: 47; Pinto 1991: 201.

Terra typica. E. pensylvanica, E. nigra types unknown. E. morio Lectotype female (designated by Werner 1945) Texas; MCZC. E. potosina Holotype (?) Huasteca de San Luis Potosí, Mexico; UNAM.

Distribution. Canada, Mexico, United States.

Location. Canada: Alberta, Manitoba, New Brunswick, Ontario, Quebec, Sakatchewan. Mexico: Chihuahua, Coahuila, Durango, Nuevo León. United States: Alabama, Arizona, Arkansas, Colorado, Connecticut, Delaware, District of Columbia, Florida, Georgia, Idaho, Illinois, Indiana, Iowa, Kansas, Kentucky, Louisiana, Maine, 
Maryland, Massachusetts, Michigan, Minnesota, Mississippi, Missouri, Montana, Nebraska, New Hampshire, New Jersey, New Mexico, New York, North Carolina, North Dakota, Ohio, Oklahoma, Pennsylvania, Rhode Island, South Carolina, South Dakota, Tennessee, Texas, Utah, Vermont, Virginia, West Virginia, Wisconsin, Wyoming.

Epicauta (Epicauta) peruensis Kaszab, 1960

Epicauta peruensis Kaszab, 1960: 407; Pinto and Bologna 2016: 205.

Terra typica. Holotype male, Tarma, Paru; SUM.

Distribution. Peru.

Location. Peru: Mountain near Tarma 3300 m; Junín.

\section{Epicauta (Epicauta) philaemata (Klug, 1825)}

Lytta philaemata Klug, 1825: 434.

Cantharis philaemata: Gemminger and Harold 1870: 2152.

Epicauta philaemata: Borchmann 1917: 69, Denier 1935b: 158, Blackwelder 1945: 484, Adams and Selander 1979: 259.

Terra typica. Syntype (s?) Brazil; ZMHU location unknown.

Distribution. Argentina, Brasil, Venezuela.

Location. Argentina: Misiones, Tucumán. Brazil: Río de Janeiro, São Paulo, Santa Catarina. Venezuela: country labeled only.

New records (Figure 7): Argentina: Misiones (Aristóbulo del Valle, Arroyo Yacutinga, Dos de Mayo) [MACN].

\section{Epicauta (Epicauta) phoenix Werner, 1944}

Epicauta phoenix Werner, 1944: 66; 1945: 443; Werner et al. 1966: 39; Pinto 1980: 55; 1991: 279 .

Terra typica. Holotype male, Phoenix, Arizona; MCZC.

Distribution. United States.

Location. United States: country labeled only.

\section{Epicauta (Epicauta) pilme Molina, 1758}

Epicauta pilme Molina, 1758.

Epicauta langei Borchmann, 1930: 95; Denier 1935b: 156; Blackwelder 1945: 483; Di

Iorio 2004: 168; Campos-Soldini et al. 2009: 4 New Synonymy.

Epicauta nigripes Borchmann, 1930: 91; Denier 1935b: 158; Blackwelder 1945: 483;

Di Iorio 2004: 170 New Synonymy.

Diagnosis. The species E. langei and E. nigripes are phentically very similar to Epicauta pilma by the combination of the following characters: body length $7-14 \mathrm{~mm}$. Tegument black. Legs with femora orange with apex black, tibiae and tarsi black. Body vestiture black, setae short, erect and minimal (6-9 setae along one mm long line) 
allowing to see tegument. Head subrectangular. Antenna subcylindrical, tapering to apex; ratios length $\left(\mathrm{L}_{\text {Ant }}\right)$ vs. width $\left(\mathrm{W}_{\text {Ant }}\right)$ of antennomere female $\left(\mathrm{L}_{\text {Ant }} / \mathrm{W}_{\text {Ant }}\right): 1.5(\mathrm{I})$, 1.3 (II), 1.6 (III), 1.2 (IV), 1 (V-VII), 2.5 (VIII-X) 3.5 (XI); male: 1.6 (I), 0.8 (II), 2 (III), 1.3 (IV-X), 2.6 (XI). Pronotum subcampaniform. For this reason, we consider these species as a single specific entity whose valid name is $E$. pilma by priority.

Terra typica. E. nigripes, Syntypes, Catamarca, Valle de Santa María; MCNBR. E. langei, Type (s?) Catamarca, La Rioja; MCNBR.

Distribution. Argentina and Chile.

Location. Argentina: Buenos Aires, Catamarca, Chubut, Mendoza, Neuquén, Río Negro, Salta. Chile: Antofagasta, Arica, Atacama, Aysén, Bío Bío, Cautín, Coaquimbo, Chiloe, Lonquihue, Maule, O’Higgins, Parincota, Tarapacá, Valdivia, Valparaiso, Villa Rica.

New records (Figure 7): Argentina: Buenos Aires (Sierra de la Ventana); Chubut (El Ojo del Epuyen); Mendoza (Godoy Cruz, Las Heras); Neuquén (Huiliche, Lago Lacar, Parque Nacional Lanin, Perdriel, Pino Hachado, Pucará, Rinconada, San Martín de los Andes); Río Negro (Bariloche, El Bolsón, Chimpay) Salta (Cachipampa, Cuesta del Obispo, Isonza). Chile: Antofagasta; Arica; Atacama; Aysén; Bío Bío; Cautín (Temuco); Coaquimbo; Chiloe; Lonquihue; Maule; O’Higgins, Parincota; Santiago de Chile; Tarapacá; Valdivia; Valparaiso; Villa Rica (Lago Caburgua) [MLPA, IADIZA, Barriga-Tuñón, FIMLA, MACN].

\section{Epicauta (Epicauta) pluvialis Borchmann, 1930}

Epicauta pluvialis Borchmann, 1830: 95; Denier 1935b 158; Bosq 1943: 11; Blackwelder 1945: 484; Viana and Williner 1974: 11; Martínez 1992: 7; Di Iorio 2004: 171; Campos-Soldini and Roig-Juñent 2015: 33.

Terra typica. Type (s?) Buenos Aires and Mendoza; MCNBR.

Distribution. Argentina.

Location. Argentina: Buenos Aires, Catamarca, Córdoba, Chubut, Entre Ríos, La Pampa, La Rioja, Mendoza, Salta, San Juan, San Luís, Río Negro.

New records (Figure 7): Argentina: Buenos Aires (Miramar); Catamarca (Hualfín, Punta Balasto, Santa María); Chubut ( Península de Valdés); Córdoba (Ascochinga, Los Cocos); Entre Ríos (Victoria); Mendoza (Barrancas, El Azufre, Challao, La Cuenca, La Agüadita, Las Heras, Lujan del Cuyo, Malargüe, Potrerillo, San Carlos Estrada, San Rafael, Villavisencio); Neuquén (Las Lajas); Río Negro (Choele Choel, Río Colorado); San Luis (Coralina) [IADIZA, Barriga-Tunón, FIMLA, MACN].

\section{Epicauta (Epicauta) proscripta Pinto, 1980}

Epicauta proscripta Pinto, 1980: 71; 1991: 279.

Terra typica. Holotype male, Jackson, Mississippi; USNM.

Distribution. United States.

Location. United States: Kansas, Mississippi, Tennessee. 


\section{Epicauta (Epicauta) pruinosa LeConte, 1866}

Epicauta pruinosa LeConte, 1866: 158; Horn 1873: 98; Maybell 1934: 327; Denier

1940: 422; Werner 1945: 471; Hatch 1965: 106; Werner et al. 1966: 44; Pinto 1991: 317.

Lytta immertia Walker, 1866: 330.

Cantharis pruinosa: Gemminger and Harold 1870: 2153.

Epicauta piceiventris Maydell, 1934: 327; Werner 1945: 472; Hatch 1965: 106.

Epicauta elongatocalcarata Maydell, 1934: 328.

Epicauta lupini Selander, 1952: 131.

Epicauta werneri Hatch, 1965: 105.

Terra typica. E. pruinosa, Lectotype female (designated by Werner 1945) Colorado Territory; MCZC. E. immerita, Holotype female, British Columbia; BMNH. E. piceiventris, Holotype male, Utah; MCZC. E. elongatocalcarata, Holotype female, Atlanta, Idaho; MCZC. E. lupini, Holotype male, Tygh Valley, Oregon; USNM. E. werneri, Holotype female

Distribution. Canada, United States.

Location. Canada: Alberta, British Columbia, Manitoba. United States: Arizona, California, Colorado, Idaho, Montana, Nebraska, New Mexico, North Dakota, Oregon, South Dakota, Utah, Washington, Wyoming.

\section{Epicauta (Epicauta) pullata (Berg, 1889)}

Lytta pullata Berg, 1889: 121; Bruch 1914: 405; Borchmann 1917: 97.

Epicauta pullata: Denier 1935b: 158; Blackwelder 1945: 484; Campos-Soldini et al. 2009: 5 .

Terra typica. Syntypes (sex?) Chacabuco, Mendoza.

Distribution. Argentina.

Location. Argentina: Buenos Aires, Mendoza.

New records (Figure 7): Argentina: Buenos Aires: Chacabuco; Mendoza (Province labeled omly) [MLPA].

\section{Epicauta (Epicauta) puncticollis Mannerheim, 1843}

Epicauta puncticollis Mannerheim, 1843: 288; LeConte 1851: 162; Horn 1873: 97;

1885: 111; Gibson 1912: 87; Wickham 1914: 492; Maydell 1934: 327; Denier 1935: 158; Moore 1937: 42; Werner 1945: 474; Parker and Wakeland 1957: 27; Middlekauff 1958: 9; Gupta 1965: 453; Hatch 1965: 106; Church 1967: 757; Pinto 1973: 968; 1975: 451; Rees 1973: 202; Cohen and Pinto 1977: 741; Ballmer 1980: 78; Pinto and Mayor 1986: 602; Pinto 1991: 333.

Cantharis puncticollis: Gemminger and Harold 1870: 2153.

Epicauta oblita: LeConte 1853: 339; Horn 1873: 97; 1885: 111; Fall 1901: 184; Maydell 1934: 327; Moore 1937: 41; Rees 1973: 198; Pinto1977: 141; Werner 1945: 475. 
Lytta oblita: LeConte 1853: 339.

Lytta puncticollis: LeConte 1853: 339.

Epicauta barberi: Werner 1944: 69; 1945: 475.

Epicauta (Epicauta) oblita: MacSwain 1956: 64.

Epicauta (Epicauta) puncticollis: MacSwain 1956: 62; Pinto 1974: 5.

Terra typica. E. puncticollis, Holotype male, "Calif. Bor."; UZMH; E. oblita Lectotype female (designated by Werner 1945) San Francisco, California; MCZC. E. barberi, Holotype male, La Panza, California; USNM.

Distribution. México, United States.

Location. Mexico: El Sauzi, Ensenada, San Antonio del Mar, Santo Tomás. United States: British Columbia, California, Montana, Nevada, San Francisco, Utah, Wayoming.

\section{Epicauta (Epicuta) punctipennis Werner, 1944}

Epicauta punctipennis Werner, 1944: 68; Dillon 1952: 391; Pinto 1975: 437; 1991: 119. Epicauta pallidilabra Dillon, 1952: 390.

Terra typica. Holotype male, Victoria, Texas; USNM.

Distribution. Canada, United States.

Location. Canada: British Columbia east to Manitoba. United States: Missouri, Nevada, New Mexico, South Dakota, Washington.

\section{Epicauta (Epicauta) purpureiceps (Berg, 1889)}

Lytta purpureiceps Berg, 1889: 123; Bruch 1914: 405; Borchmann 1917: 97.

Cantharis purpureiceps: Chmapion 1899: 186.

Epicauta purpureiceps: Borchmann 1917: 97.

Epicauta kraussi var. purpureiceps: Denier 1935: 156; 1940: 156.

Terra typica. Holotype female (?) Cordoba, Argentina; MLPA.

Distribution. Argentina, Brazil.

New records (Figure 7): Argentina: Córdoba, Santa Fe (provinces labeled only) [MLPA].

Epicauta (Epicauta) rehni Maydell, 1934

Epicauta rehni Maydell, 1934: 329; Denier 1940: 422; Werner 1945: 485; 1949: 107; 1955: 5; Werner et al. 1966: 41; Pinto 1972: 254; 1991: 153.

Terra typica. Holotype female, Schaeffer Canyon, Bobaquivari Mts., Arizona; ANSP. Distribution. United States.

Location. United States: Arizona, New Mexico.

\section{Epicauta (Epicauta) rileyi Horn, 1874}

Epicauta rileyi Horn, 1874: 43; 1891: 43; Van Dyke 1929: 129; Maydell 1934: 329, 330; Denier 1935: 159; Pinto 1991: 154. 
Terra typica. Lectotype female: (designated by Werner 1945) Arizona; MCZC.

Distribution. Mexico, United States.

Location. Mexico: Sonora. United States: Arizona.

Epicauta (Epicauta) riojana (Fairmaire, 1892)

Cantharis griseonigra var. riojana Fairmaire, 1892: 252.

Lytta griseonigra var. riojana: Bruch 1914: 405.

Epicauta griseonigra var. riojana: Borchmann 1917: 76; Denier 1935b: 156; Blackwelder 1945: 483.

Epicauta riojana: Campos-Soldini 2011: 582.

Terra typica. Unknown.

Distribution. Argentina.

Location. Argentina: Córdoba; Entre Ríos, La Rioja, Salta, Tucumán.

New records (Figure 8): Argentina: Córdoba (Mansilla); Entre Ríos (Villa Elisa); Salta (Santa Salta Forestal); Tucumán (Gobernador Garmendia) [IADIZA, Barriga-Tunón].

\section{Epicauta (Epicauta) rubella Denier, 1940}

Epicauta rubella Denier, 1940: 182; Blackwelder 1945; Campos-Soldini et al. 2009: 6;

Campos-Soldini and Roig-Juñent 2015: 35.

Terra typica. Holotype male, Paraguay: Puerto Max; MLPA. Allotype female, Salta: Esteco (MLPA).

Distribution. Argentina, Paraguay.

Location. Argentina: Salta: Departamento Salta Forestal. Paraguay: Puerto Max.

Epicauta (Epicauta) rufipennis (Chevrolat, 1834)

Canthris rufipennis Chevrolet, 1834: 80; Dugès 1870: 127.

Cantharis ocreaceipennis Dugès, 1870: 164.

Cantharis rufipennis: Gemminger and Harold 1870: 2153.

Cantharis ochreipennis Dugès, 1877: 57.

Epicauta rufipennis: Dugès 1889: 60; Champion 1892: 408; Denier 1935; 159; Blackwelder 1945: 484; Pinto 1991: 256.

Terra typica. E. rufipennis Holotype (sex?) Mexico; probably in UZMH.

Distribution. Guatemala, Mexico.

Location. Guatemala: Guatemala, Purulha, Baja Vera Paz. Mexico: Chihuahua, Distrito Federal, Guanajuato, Guerrero, Hidalgo, Jalisco, Mexico, Michoacán, Morelos, Nayarit, Oaxaca, Puebla, Sinaloa, Veracruz.

\section{Epicauta (Epicauta) rutilifrons Borchmann, 1930}

Epicauta rutilifrons Borchmann, 1930: 91; Blackwelder 1945: 484; Martínez 1992: 8;

Campos-Soldini et al. 2009: 6; Campos-Soldini and Roig-Juñent 2015: 40. 
Terra typica. Syntypes, Ledesma, Jujuy and Senillosa; MACN.

Distribution. Argentina, Brazil.

Location. Argentina: Jujuy, Salta. Bolivia. Brazil: Matto Grosso.

New records: Bolivia (country labeled only) (FIMLA, MACN).

Epicauta (Epicauta) sanguinicollis (LeConte, 1853)

Lytta sanguinicollis LeConte, 1853: 344.

Cantharis sanguinicollis: Gemminger and Harold 1870: 2153.

Epicauta sanguinicollis: Horn 1873: 103; 1874: 37; Denier 1935: 159; Werner 1945:

473; Selander 1984: 3; Pinto 1991: 334.

Terra typica. Neotype female (designated by Pinto 1991) Florida; MCZC.

Distribution. United States.

Location. United States: Florida, Georgia, South Carolina.

Epicauta (Epicauta) sanguinithorax (Haag-Rutenberg, 1880)

Lytta sanguinithorax Haag-Rutenberg, 1880: 34.

Epicauta sanguinithorax: Borchmann 1917: 82.

Terra typica. Holotype male, Peru; ZSBS.

Distribution. Argentina, Peru.

Location. Argentina: La Rioja. Peru.

New records (Figure 8): Argentina: La Rioja (Guayapa) (FIMLA).

Epicauta (Epicauta) saopaoloana Kaszab, 1960

Epicauta saopaoloana Kaszab, 1960: 285.

Terra typica. Allotype and Paratype female, São Paulo, Brazil; Sammlung des Museums G. Frey. Holotype male, Quimadas, Brazil; SUNM.

Distribution. Brazil.

Location. Brazil: São Paulo.

Epicauta (Epicauta) semivittata (Fairmaire, 1875)

Cantharis semivittata Fairmaire, 1875: 200.

Cantharis hemigramma Mäklin, 1875: 632.

Cantharis virgata Burmeister, 1881: 25.

Epicauta semivittata: Bruch 1914: 404; Borchmann 1917: 82; Denier 1935b: 159;

Bosq 1934: 327; 1943: 12; Blackwelder 1945: 484; Viana and Williner 1974: 16;

Di Iorio 2004: 171; Campos-Soldini and Roig-Juñent 2015: 40.

Pyrota virgata: Borchmann 1917: 69.

Terra typica. Unknown.

Distribution. Argentina, Chile, Uruguay. 


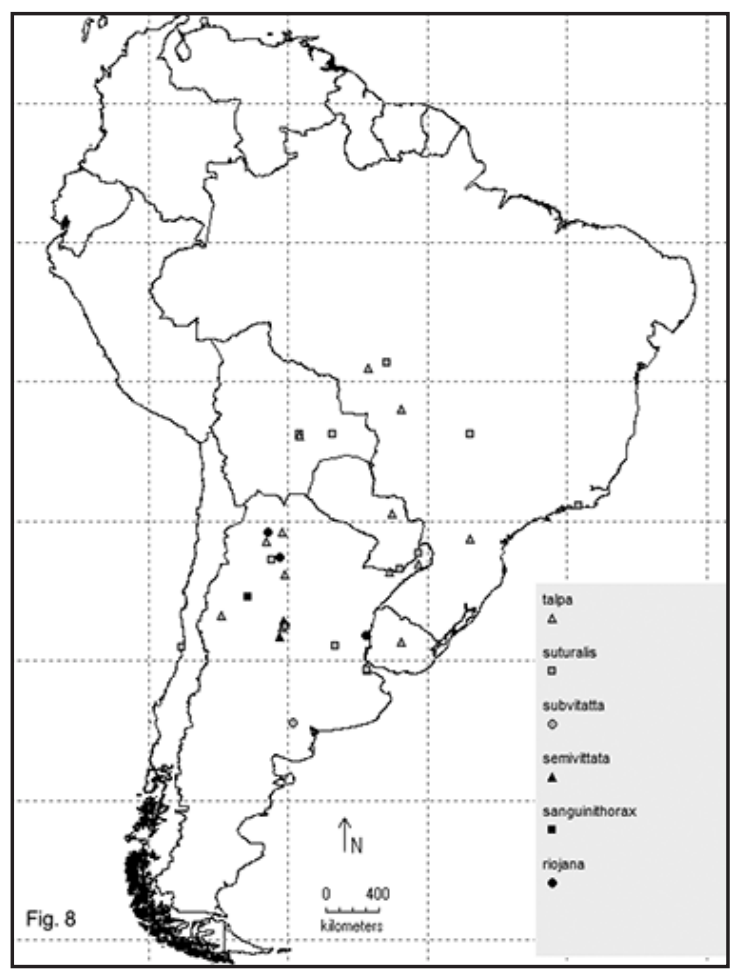

Figure 8. New distribution records for: E. riojana; E. sanguinithorax; E. semivittata; E. subvittata; E. suturalis; and E. talpa.

Location. Argentina: Buenos Aires, Catamarca, Córdoba, Corrientes, Jujuy, La Pampa, Mendoza, Santa Fé, San Luis, Tucumán. Uruguay: Carrasco.

New records (Figure 8): Argentina: Buenos Aires (province labeled only); Córdoba (Calamuchita, El Sauce) [Barriga-Tuñón, MACN].

\section{Epicauta (Epicauta) senilis Werner, 1949}

Epicauta senilis Werner, 1949: 102; Werner et al. 1966: 44; Pinto 1991: 155.

Epicauta candidata: Dillon 1952: 395.

Terra typica. Holotype male, Luna Co., New Mexico Pinto (1991) indicates that the type is unknown.

Distribution. Mexico, United States.

Location. Mexico: Chihuahua, Durango. United States: Arizona, New Mexico, Texas.

\section{Epicauta (Epicauta) sericans LeConte, 1866}

Lytta sartorii Haag-Rutenberg, 1880: 56.

Epicauta sericans LeConte, 1866: 158; Horn 1873: 98; 1891: 43; Blair 1921: 281;

Milliken 1921: 6; Carruth 1931: 52; Denier 1935: 159; Werner 1945: 469; Vau- 
rie 1950: 29; Dillon 1952: 385; Parker and Wakeland 1957: 26; Burke 1963: 53;

Werner et al. 1966: 44; Church 1967: 756; Goeden 1971: 42; Rees 1973: 205;

Arnold 1976: 26; Selander 1952: 131; 1982: 427; Capinera et al. 1985: 1054.

Cantharis sericans: Gemminger and Harold 1870: 2153.

Epicauta (Epicauta) callosa: MacSwain 1956: 56.

Terra typica. E. sericans, Lectotype female (designated by Werner 1945) Kansas; MCZC. E. sartorii, types from "Mexico, Mirador", location of syntypes is unknown.

Distribution. Canada, Mexico, United States.

Location. Canada: Alberta, Saskatchewan. Mexico: Chihuahua, Coahuila, Durango, Hidalgo, Jalisco, Mexico, Nuevo León, Puebla, Querétero, San Luis Potosí, Tamaulipas, Veracruz. United States: Alabama, Arkansas, Colorado, Georgia, Indiana, Iowa, Kansas, Kentucky, Louisiana, Minnesota, Missouri, Montana, Nebraska, New Mexico, North Dakota, Oklahoma, South Dakota, Tennessee, Wyoming.

\section{Epicauta (Epicauta) singularis Champion, 1892}

Epicauta singularis Champion, 1892: 427; Denier 1935: 159; Blackwelder 1945: 484;

Vauri 1950: 30; Werner 1955: 5; Pinto 1982: 407; 1991: 157.

Terra typica. Lectotype male (designated by Pinto 1982) Monterrey, Nuevo León; BMNH.

Distribution. Mexico, United States.

Location. Mexico: Nuevo León, Texas.

Epicauta (Epicauta) solaniperda Denier, 1940

Epicauta solaniperda Denier, 1940: 422.

Terra typica. Holotype male and Allotype female, Huancayo, Peru; MLPA type locality unknown.

Distribution. Peru.

\section{Epicauta (Epicauta) straba Horn, 1891}

Epicauta straba Horn, 1891: 42; Fall 1901: 184; Van Dayke 1929: 129; Denier 1935:

159; Moore 1937: 42; Werner 1945: 483; 1955: 5; Pinto 1972: 254; 1991: 158.

Terra typica. E. straba Lectotype (designated by Werner 1945) San Bernardino California; MCZC. E. foxi Holotype female, Jacumba, San Diego Co., California.

Distribution. Mexico, United States.

Location. Mexico: Baja California Norte. United States: California.

\section{Epicauta (Epicauta) strigata (Gyllenhal, 1817)}

Lytta strigata Gyllenhal, 1817: 18.

Cantharis strigata: Fischer 1827: 19.

Epicauta strigata: Borchmann 1917: 83; Martínez 1955: 58. 
Terra typica. Type (s?) from Brazil; ZMU, type loction unknown. Distribution. Brazil.

\section{Epicauta (Epicauta) strigosa (Gyllenhal, 1817)}

Lytta strigosa Gyllenhal, 1817: 18; LeConte 1853: 314.

Cantharis strigosa: Fischer 1827: 19.

Cantharis nigricornis Melsheimer, 1846: 53.

Cantharis strigosa: Gemminger and Harold 1870: 2154.

Epicauta strigosa: Horn 1873: 97; 1875: 153; Blatchely 1910: 1361; Ulke 1902: 54;

Sherman 1913: 246; Mutchler and Weiss 1924: 9, 18; Denier 1935: 159; Werner 1945: 467; 1957: 97, 98; Dillon 1952: 384; Selander 1952: 131; 1984: 3; Staines 1983: 48; Pinto 1991: 341.

Terra typica. E. strigosa, type (s?) presumably in ZUMU. E. nigricornis Lectotype (sex?) (designated by Werner 1945) Alabama; MCZC.

Distribution. United States.

Location. United States: Alabama, Connecticut, District of Columbia, Florida, Georgia, Louisiana, Massachusetts, Mississippi, New Jersey, New York, North Carolina, Rhode Island, South Carolina, Texas, Virginia.

\section{Epicauta (Epicauta) stuarti LeConte, 1868}

Epicauta stuarti LeConte, 1868: 54; Horn 1873: 101; Maydell 1934: 333; Werner 1945: 462; 1955: 3,4; Selander 1954: 24; Werner et al. 1966: 42; Pinto 1991: 160.

Terra typica. Lectotype female (designated by Werner 1945) Ft. Union, New Mexico; MCZC.

Distribution. United States.

Location. United States: Arizona, Colorado, Kansas, Nebraska, New Mexico, Oklahoma, Texas, Wyoming.

\section{Epicauta (Epicauta) subatra Dugès, 1889}

Epicauta subatra Dgès, 1889: 72; Champion 1892: 421; Denier 1935: 159; Blackwelder 1945: 484; Pinto 1991: 205.

Terra typica. Holotype female, Mexico (Pinto 1991) indicates that the types is probably lost).

Distribution. Mexico.

Location. Mexico: Hidalgo, Nuevo León, Puebla.

\section{Epicauta (Epicauta) subvittata (Erichson, 1848)}

Lytta (Epicauta) subvittata Erichson, 1848: 566.

Lytta sublineata: Haag-Rutenberg 1880: 53.

Epicauta subvittata: Borchmann 1917: 84. 
Terra typica. Type (s?) Guayana (=British Guaiana); ZMHU, type location unknown. Distribution. Brazil, Guayana.

Location. Brazil: São Paulo.

New records (Figure 8): Brazil: São Paulo (state labeled ony) [MLPA].

\section{Epicauta (Epicauta) suturalis (Haag-Rutenberg, 1880)}

Lytta albicincta Haag-Rutenberg, 1880: 23. New Synonymy.

Cantharis suturalis Germar, 1821: 247; Gemminger and Harold 1870: 2154.

Epicauta suturalis: Bruch 1911: 405; Borchmann 1917: 84; Denier 1935: 159; Black-

welder 1945: 484.

Epicauta albicincta: Borchmann 1917: 70; Denier 1935: 152; Blackwelder 1945: 482.

Diagnosis. Epicauta sutrualis is phenetically alike to E. albicincta by the combination of the following characters: body length large $(>17 \mathrm{~mm})$. Tegument black. Body vestiture cinereous, setae short, decumbent, dense (28-34 setae along one mm long line). Antenna flattened dorsoventraly in both sexes; ratios length $\left(\mathrm{L}_{\text {Ant }}\right)$ vs. width $\left(\mathrm{W}_{\text {Ant }}\right)$ of antennomere $\left(\mathrm{L}_{\mathrm{Ant}} / \mathrm{W}_{\mathrm{Ant}}\right)$ in female: 3.75 (I), 2.33 (II), 6 (III), 4.6 (IV) 4 (V-X) 4.3 (XI); in male 3 (I), 2 (II), 5 (III), 3.3 (IV), 3 (V-VIII), 5 (IX-XI).

Terra typica. Type (s?) Merida, Venezuela; ZSBS.

Distribution. Argentina, Bolivia, Brazil, Paragauay, Venezuela, Uruguay.

Location. Argentina: Buenos Aires, Misiones, Jujuy, Salta, Tucumán. Chile: Valparaiso. Bolivia: Chiquitos; Cuatro Ojos. Brazil: Campo Bello; Goyaz (Río Verde, Yathay); Matto Grosso; Río de Janeiro; São Paulo. Paraguay: Asunción. Uruguay: Canelones (La Floresta). Uruguay: Cerro Largo.

New records (Figure 8): Argentina: Buenos Aires (Cerro Largo); Misiones (Alto Paraná, Puerto Victoria); Sante Fe (Casilda, Rosario); Tucumán. Chile: Valparaiso (province labeled only). Bolivia: Santa Cruz (Chiquitos, Cuatro Ojos). Brazil: Goyaz (Río Verde,); Mato Grosso (state labeled only); Río de Janeiro (Río de Janeiro). Paraguay: Asunción. Uruguay: Cerro Largo (Cuhilla de Melo) [MLPA, FMILA, MACN].

\section{Epicauta (Epicauta) talpa (Haag-Rutenberg, 1880)}

Lytta talpa Haag-Rutenberg, 1880: 32.

Cantharis talpa: Berg 1881: 306.

Epicauta talpa: Beauregard 1890: 511; Bruch 1911: 404; Borchmann 1917: 85; Denier 1935: 160; Blackwelder 1945: 484; Martínez 1992: 8.

Terra typica. Type (s?) Cordoba; presumably in ZSBS.

Distribution. Argentina, Bolivia, Brazil, Paraguay, Uruguay.

Location. Argentina: Chaco, Córdoba, Corrientes, Misiones, Salta, Santiago del Estero. Bolivia: Andrés Ibáńez (Santa Cruz de la Sierra). Brazil: Mato Grosso (Rancho Grande, San Antonio das Barras, Utiariti); Paraná (Prudentrópolis). Paraguay: Concepción (Río aquidaban); San Pedro (Cororo). Uruguay: Tacuarembó (San Gregorio de Polanco). 
New records (Figure 8): Argentina: Córdoba (Alta Gracia, Cruz del Eje, La Merced); Corrientes (Ituzaingó); Misiones (Dos de Mayo); Salta (Capiazutí, La Viña); Santiago del Estero (Río Salado). Bolivia: Andrés Ibáńez (Santa Cruz de la Sierra). Brazil: Mato Grosso (San Antonio das Barras, Utiariti); Parana (Prudentópolis). Paraguay: San Pedro (Cororo). Uruguay: Tacuarembó (San Gregorio de Polanco) [MLPA, MACN].

Epicauta (Epicauta) tamara Adams \& Selander, 1979

Epicauta dugesi: Werner, 1957: 107.

Epicauta tamara Adams \& Selander, 1979: 246; Pinto 1991: 349.

Terra typica. Holotype male, Culicán, Sinaloa, Mexico; AMNH.

Distribution. Mexico.

Location. Mexico: Sinaloa, Sonora.

Epicauta (Epicauta) tarasca Pinto, 1991

Epicauta tarasca Pinto, 1991: 207.

Terra typica. Holotype male, 3 km E Quiroga, Michoacán, Mexico; CASC.

Distribution. Mexico.

Location. Mexico: Jalisco, Michoacán, Morelos, Oaxaca.

\section{Epicauta (Epicauta) temexa Adams \& Selander, 1979}

Epicauta vittata: Dugès 1889: 87.

Epicauta lemniscata: Champion 1892: 415; Snow 1906: 149; Dillon 1952: 398 (in part). Epicauta temexa Adams \& Selander, 1979: 248; Ray et al. 1989: 187.

Terra typica. Holotype male, Pearsall, Frio Co., Texas; AMNH.

Distribution. Mexico, United States.

Location. Mexico: Coahuila, Nuevo León, San Luis Potosí, Tamaulipas, Veracruz. United States: Texas.

\section{Epicauta (Epicauta) tenebrosa Werner, 1949}

Epicauta pedalis: Horn 1894: 356 (in part); Werner 1945: 440 (in part).

Epicauta tenebrosa Werner 1949: 93; Werner et al. 1966: 38; Pinto 1991: 259.

Terra typica. Holotype male, Tucson, Arizona; MCZC.

Distribution. Mexico, United States.

Location. Mexico: Sonora. United States: Arizona.

Epicauta (Epicauta) teresa Mathieu, 1983

Epicauta (Epicauta) teresa Mathieu, 1983: 156.

Epicauta teresa: Pinto 1984: 378; 1991: 268. 
Terra typica. Holotype male, $10 \mathrm{~km}$ northesast of Cintalapa, Chiapas, Mexico; CASC. Distribution. Mexico.

Location. Mexico: Chiapas, Oaxaca.

\section{Epicauta (Epicauta) tricostata (Werner, 1943)}

Pleuropompha tricostata Werner, 1943: 32; 1945: 426; Vaurie 1950: 39; Dillon 1952:

379; MacSwain 1956: 66; Werner et al. 1966: 54; Pinto 1973: 957; 1977: 136;

Selander 1981: 780.

Epicauta tricostata: Pinto 1984: 381; 1991: 231; Pinto and Mayor 1986: 602.

Terra typica. Holotype male, Presidio, Texas; USNM.

Distribution. Mexico, United States.

Location. Mexico: Coahuila, San Luis Potosí. United States: Arizona, New Mexico, Texas.

\section{Epicauta (Epicauta) tristis (Mäklin, 1875)}

Cantharis tristis Mäklin, 1875: 630.

Lytta lugubris Haag-Rutenberg, 1880; Borchmann 1917: 95.

Epicauta tristis: Beauregard 1890: 512; Denier 1935: 160; Blackwelder 1945: 484; Di

Iorio 2004: 172.

Epicauta lugubris: Denier 1935: 156; Blackwelder 1945: 483 (Nudum erat nomen)

Epicauta luguberrima Denier, 1935: 156 New Synonymy.

Diagnosis. Epicauta tristis and E. lugubris are alike regarding several features, such as: body length 10-12 mm. Tegument black; head with reddish patch on front. Superficial nonparallel sculpturing: head and pronotum punctuate. Body vestiture dorsally black, marginal and ventraly cinereous; seate dense (28-34 setae along one $\mathrm{mm}$ long line). Antenna similar in both sexes; ratios length $\left(\mathrm{L}_{\text {Ann }}\right)$ vs. width $\left(\mathrm{W}_{\text {Ant }}\right)$ antennomere $\left(\mathrm{L}_{\text {Ant }} / \mathrm{W}_{\text {Ant }}\right): 2.3$ (I), 1.6 (II), 3.5 (III-XI), in male 2.3 (I), 1.6 (II), 3.6 (III), 2.3 (IV-XI). For this reason, we consider these species (E. tristis and E. lugubris) as a single specific entity whose valid name is $E$. tristis by priority.

Terra typica. Lytta lugubris Type (s?) Brazil; ZSBS.

Distribution. Argentina, Bolivia, Brazil.

Location. Argentina: Córdoba, Jujuy, Misiones, Mendoza, San Juan, San Luis, Salta. Bolivia: Santa Cruz de la Sierra. Brazil.

New records (Figure 9): Argentina: Salta (province labeled only) [FIMLA].

\section{Epicauta (Epicauta) unilineata Champion, 1892}

Epicauta unilineata Champion, 1892: 415; Denier 1935: 160; Blackwelder 1945: 484;

Adams and Selander 1979: 250.

Terra typica. Lectotype male (designated by Admas and Selander 1979) San Jerónimo, Guatemala; location unknown. 
Distribution. El Salvador, Guatemala, Mexico.

Location. El Salvador: La Unión, San Salvador, Santa Ana. Guatemala: Baja Verapaz, Guatemala. Mexico: Guerrero, Veracruz.

\section{Epicauta (Epicauta) ventralis Werner, 1945}

Epicauta ventralis Werner, 1945: 444; Pinto 1975: 430; 1980: 48.

Epicauta normalis: Werner 1944: 65 (in part); 1945: 442 (in part); Hatch 1965: 105;

Werner et al. 1966: 38 (in part).

Terra typica. Holotype male, Walsenburg, Colorado; FMNH.

Distribution. Canada, United States.

Location. Canada: Alberta, Saskatchewan. United States: Arizona, California, Colorado, Idaho, Kansas, Montana, Nebraska, Nevada, New Mexico, North Dakota, Oregon, South Dakota, Utah, Washington, Wyoming.

\section{Epicauta (Epicauta) vicina (Haag-Rutenberg, 1880)}

Lytta vicina Haag-Rutenberg, 1880: 27.

Epicauta vicina: Borchmann 1917: 85; Denier 1935: 160; Blackwelder 1945: 484.

Epicauta wagneri Pic, 1916: 10; Denier 1935: 160; Blackwelder 1945: 484 New Synonymy.

Diagnosis. The tegument color (pale brown with head and pronotum black); body vestiture dense (28-34 setae along one $\mathrm{mm}$ long line); and seate elongate, disheveled apparance and yellow of $E$. vicina are similar to E. wagneri. For this reason, we consider this species as a single specific entity whose valid name is $E$. vicina by priority.

Terra typica. Type (s?) Brazil; "Mus. Vind. and Haag-Rutenberg (in part); ZSBS.

Distribution. Argentina, Brazil, Paraguay.

Location. Argentina: Chaco; Corrientes; Entre Ríos; Misiones; Santiago del Estero. Brazil: Guaiaba. Paraguay: Villa Rica.

New records (Figure 9): Argentina: Entre Ríos (Concordia, Paraná); Corrientes (Chavarría); Misiones (Pindapoy); Santiago del Estero (Río Dulce). Paraguay: Guairá (Villa Rica) [MLPA].

\section{Epicauta (Epicauta) vidua (Klug, 1825)}

Lytta vidua Klug, 1825: 11.

Causima luctuosa Dejean, 1837: 248.

Causima lugubris Dejean, 1837: 248.

Cantharis vidua: Burmeister 1881: 23; Berg 1881: 303; Gemminger and Harold 1870: 2155.

Causima vidua: Denier 1935: 161; Blackwelder 1945: 484; Di Iorio 2004: 165.

Distribution. Argentina, Bolivia, Brazil. 
Location. Argentina: Córdoba, Corrientes, Misiones (General Belgrano, Puerto Aguirre, and Santa Ana). Bolivia. Brazil: Parana.

New records (Figure 9): Argentina: Córdoba (Salsacate); Misiones (Aristóbulo del Valle, Arroyo Cañapirú, Arroyo Uruguaí, Puerto Aguirre, Ruta Nac. 12, Santa Ana, Salto Encantado). Brazil: Parana (state labled only) [IADIZA, MLPA, FIMLA, MACN].

\section{Epicauta (Epicauta) vittata (Fabricius, 1775)}

Typical race

Lytta vittata Fabricius, 1775: 260.

Meloe vittatus: Thunberg 1781-1791: 109.

Meloe chapmani: Woodhouse 1800: 214.

Cantharis vittata: Gemminger and Harold 1870: 2155.

Epicauta vittata: Horn 1873: 100; Denier 1935: 160; Staig 1940: 139; Werner 1945:

464; Admas and Selander 1979: 240; Pinto 1991: 352.

Epicauta lemniscata: Putnam 1876: 173; Werner 1945: 463 (in part); Blodgett and

Higgins 1988: 1456.

Epicauta (Epicauta) vittata: MacSwain 1956: 49.

Lemniscate race

Lytta lemniscata Fabricius, 1801: 79.

Cantharis lemnsicata: Fischer 1827: 19.

Epicauta lemniscata: Schwarz 1878: 464; Chitenden 1903: 115; Swingle and Mayer

1944: 141; Frost 1964: 140; Kirk 1969: 63 (in part).

Epicauta vittata: Watson 1917: 64.

Epicauta (Epicauta) vittata: Kirk 1970: 61 (in part).

Terra typica. E. vittata, Lectotype (sex?) (designated by Werner 1945) GUHC. E. chapmani, Types, Bucks Co., Pennsylvania (apparently destroyed). E lemniscata, Type male, "Carolina"; UZMH.

Distribution. Typical race: Canada, United States. Lemniscate race: United States.

Location. Typical race: Canada: Ontario, Quebec. United States: Alabama, Arkansas, Connecticut, Delaware, District of Columbia, Florida, Georgia, Illinois, Indiana, Iowa, Kansas, Kentucky, Louisiana, Maryland, Massachusetts, Michigan, Minnesota, Mississippi, Missouri, Nebraska, New Jersey, New York, North Carolina, Ohio, Oklahoma, Pennsylvania, Rhode Island, South Carolina, South Dakota, Tennessee, Virginia, West Virginia. Lemniscate race: United States: Florida, Georgia, South Carolina.

\section{Epicauta (Epicauta) vitticollis (Haag-Rutenberg, 1880)}

Lytta vitticollis Haag-Rutenberg, 1880: 52.

Epicauta canoi Dugès, 1889: 86.

Epicauta vitticollis: Champion 1892: 414; Denier 1935: 160; Blackwelder 1945: 484;

Adams and Selander 1979: 250; Pinto 1991: 353. 


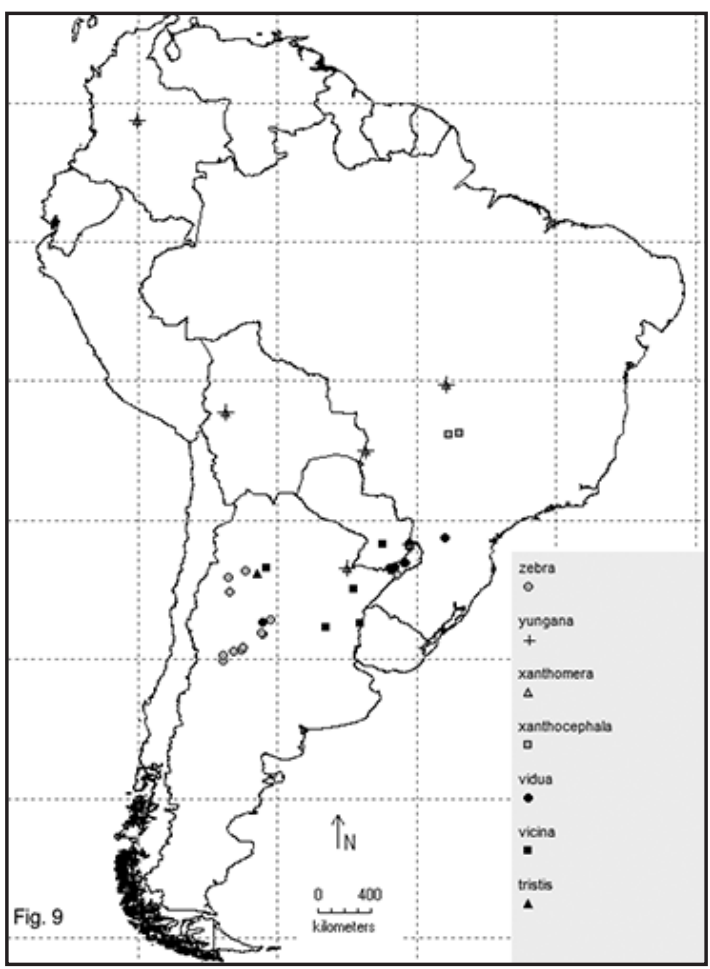

Figure 9. New distribution records for: E. tristis; E. vicina; E. vidua; E. xanthocephala; E. xanthopterus; E. yungana; and E. zebra.

Terra typica. E. vitticollis, Lectotype male, "Guatimal./Samml. Haag-Rutenberg/" (desgnated by Pinto 1991) ZSMC. E. canoi, Types, Veracruz, Mexico; apparently lost.

Distribution. Belize, Guatemala, Honduras, Mexico, Nicaragua.

Location. Belize: Corazal, Orange Walk, Toledo. Guatemala: Alta Verapaz, Petén. Honduras: Atlantida, Colón, Francisco Morazán, Yoro. Mexico: Chiapas, Oaxaca, Quintana Roo, Tabasco, Yucatán, Veracruz. Nicaragua: Río San Juan.

\section{Epicauta (Epicauta) weyrauchi Kaszab, 1960}

Epicauta weyrauchi Kaszab, 1960: 402. Pinto and Bologna 2016: 206.

Terra typica. Holotype and Paratype male, Andahuaylas, Peru. SMB.

Distribution. Peru.

Location. Peru: Apurimac.

\section{Epicauta (Epicauta) wheeleri Horn, 1873}

Epicauta wheeleri Horn, 1873: 101, 107; Werner 1945: 486; 1949: 107; 1955: 5; Werner et al. 1966: 41; Pinto 1972: 256, 1973: 968; 1991: 161. 
Terra typica. six syntypes, Arizona; MCZC.

Distribution. United States.

Records. United States: Arizona, California, Nevada, Utha.

Epicauta (Epicauta) willei Denier, 1940

Epicauta willei Denier, 1940: 422; Campos-Soldini et al. 2009: 6; Pinto and Bologna 2016: 206.

Terra typica. Holotype male, Allotype female, Paratypes male and female, Huancayo, Peru; MLPA.

Distribution. Peru.

Location. Peru: Cuzco: Sicuani, Huancayo and Junín.

Epicauta (Epicauta) xanthomera (Fischer, 1827)

Cantharis xanthomeros Fischer, 1827: 19; Geminnger and Harold 1870: 2155.

Epicauta xanthomera: Denier 1935: 169.

Terra typica. Unknown.

Distribution. Argentina, Brazil, Uruguay.

Location. Argentina: Córdoba, La Rioja. Brazil: Mina Gerais; Río de Janeiro. Uruguay: Cerro Largo.

New records (Figure 9): Argentina: Córdoba (Calamuchita, Unquillo); La Rioja (province labeled only). Brazil: Mina Gerais (Belo Horizonte); Río de Janeiro (state labeled only). Uruguay: Cerro Largo (Cañada de los Burros) [MLPA, MACN].

Epicauta (Epicauta) xanthocephala (Klug, 1825)

Lytta xanthocephala Klug, 1825: 434.

Cantharis xanthocephala: Fischer 1827: 20.

Epicauta xanthocephala: Borchmann 1917; Adams and Selander 1979: 260.

Terra typica. Type (s?) Brazil; ZMHU, type locality unknown.

Distribution. Brazil.

Location. Brazil: Goiáz.

New records (Figure 9): Brazil: Goiáz (Jatahy, Río Verde) [MLPA].

Epicauta (Epicauta) yungana Denier, 1935

Epicauta yungana Denier, 1935: 160; 1940: 422; Admas and Selander 1979: 260.

Terra typica. Holotype male, Coroico, 1300-1700 m, Nor Yungas, Bolivia; MLPA.

Distribution. Argentina, Bolivia, Brazil, Guayana.

Location. Argentina: Chaco; Córdoba; Misiones. Bogotá: Campobello. Bolivia: Coroico; La Paz; Puerto Aguirre. Brazil: Itatiaya. Guayana: Puerto Pariacabo. 
New records (Figure 9): Argentina: Chaco (Resistencia); Misiones (Salto Iguazú). Bogotá: Campobello. Bolivia: Coroico, Puerto Aguirre. Brazil: Itaiaya. Guayana: Puerto Pariacabo [MLPA].

Epicauta (Epicauta) zebra (Dohrn, 1876)

Cantharis zebra Dohrn, 1876: 411.

Lytta albovittata Haag-Rutenberg, 1880: 29.

Cantharis albovittata: Burmeister 1881: 23; Berg 1881: 303.

Epicauta somnolenta Beauregard, 1890: 510; Bruch 1914: 404; Borchmann 1917: 83;

Bosq 1934: 12, Viana and Williner 1974: 11.

Epicauta zebra: Denier 1935b: 161; 1940: 422; Hayward 1942: 23; Blackwelder 1945:

484; Adams and Selander 1979: 162; Martínez 1992: 8; Di Iorio 2004: 172; Campos-Soldini 2011: 583.

Terra typica. Cantharis zebra, Type (s?) Córdoba, Argentina, present location unknown. Lytta albovittata, Syntypes Córdoba, Argentina; presumably in ZSBS.

Distribution. Argentina.

Location. Argentina: Catamarca, Córdoba, La Rioja, Mendoza, Misiones, Salta, San Juan, San Luis, Santiago del Estero, Tucumán.

New records (Figure 9): Argentina: Catamarca (Andalgalá); Córdoba (La Falda, La Paz, San Javier); La Rioja (Chilecito, Tinogasta); Mendoza (Desaguadero, Nacuñan, Santa Rosa); Misiones (Santa María); San Luis (Balde, San Gerónimo) [IADIZA, Barriga-Tunón; FIMLA, MACN]

\section{Epicauta (Epicauta) zischkai Martinez, 1955}

Epicauta zischakai Martínez, 1955: 55; Kaszab 1960: 404; Di Iorio 2004: 172; Pinto and Bologna 2016: 206.

Terra typica. Holotype male, Allotype female, 15 Paratype male and nine Paratype female in FML. 15 Paratype male and three Paratype female in the Universidad de Vermont in Burlington, Vermont, USA. One Paratype (sex?) in Munich Muesum, Gemany.

Distribution. Bolivia, Peru.

Location. Bolivia Chapare, Cochabamba. Peru: Lago Titicaca, Puno.

\section{Acknowledgements}

We would like to extend our sincerest thanks and appreciation to Ivana Herdt for editing the manuscript. We would like to thank to the anonymous referees for their very useful comments and suggestions. We would like to thank the following people for access to specimens: Instituo Argentino de Investigaciones de las Zonas Áridas, Mendoza Argenitna (IADIZA, Sergio Roig-Juñent). Fundación e Instituto Miguel Lillo, San 
Miguel de Tucumán, Argentina (FIMLA, Gustavo Scrocchi). Museo de La Plata, La Plata, Argenitna (MLPAC, Analía Lanteri). Museo Argentino de Ciencias Naturales "Bernardino Rivadavia", Buenos Aires, Argentina (MACN, Arturo Roig-Alsina). Private collection Barriga-Tuñón curicó (Chile).

\section{References}

Adams L, Selander RB (1979) The biology of blister beetles of the vittata group of the genus Epicauta (Coleoptera, Meloidae). Bulletin of the American Museum of Natural History 162: 139-266.

Agafitei NJ, Selander RB (1980) The first instar larvae of the vittata group of the genus Epicauta (Coleoptera: Meloidae). Journal of Kansas Entomology Society 53: 1-26.

Berg FGC (1881) Revision der argentinischen Arten der Gattung Cantharis. Entomologische Zeitung Heraus Entomologische Vereine zu Stetting 42: 301-309.

Berg FGC (1883) Doce heterómeros nuevos de la fauna Argentina. Annales de la Sociedad Científica Argentina 15: 66-78.

Blackwelder RE (1945) Checklist of the coleopterous insects of Mexico, Central America, the West Indies, and South America. Part 3. US National Museum Bulletin 185: 343-550.

Borchmann F (1917) Coleopterorum Catalogus Pars 69, Meloidae, Cephaloidae. Berlin, 208 pp. Borchmann F (1930) Alleculidae y Meloidae. Revista de la Sociedad Entomológica Argentina 3: 85-100.

Borchmann F (1937) Neue Alleculiden aus dem Deutscher Entomologischen Institut, Berlin.

Borchmann F (1940) Neue Meloiden-Arten (Col.) II. Mitteilungen der Münchner.

Bologna MA (1991) Coleoptera Meloidae. Fauna d'Italia. XXVIII. Calderini, Bologna, 541 pp.

Borchmann F (1952) Meloidae, Lagriidae, Alleculidae und Nilionidae (Col.). In: Titschack E (Ed.) Beiträge zur Fauna Peru 2: 300-314.

Bosq JM (1934) Primera lista de los coleópteros de la República Argentina dañinos a la agricultura. Boletín del Ministerio de Agricultura de la Nación 36: 313-346.

Bosq JM (1943) Segunda lista de los coleópteros de la República Agentina dañinos a la agricultura. Boletín del Ministerio de Agricultura de la Nación 4 (1942): 1-80.

Brèthes J (1910) Coleópteros Argentinos y Bolivianos. Annales de la Sociedad Científica Argentina 69: 205-227.

Brèthes J (1915) Notas entomológicas. Bulletin of the Society Physics 8: 583-584.

Burmeister HCC (1881) Die argentinischen Canthariden. Stettiner Entomologische Zeitung 42: 20-35.

Campos Soldini MP, Suárez SM, Lanteri AA (2009) Types of Meloidae (Coleoptera: Tenebronoidea) housed at the Museo de La Plata entomological collection (Argentina) Revista de la Sociedad Entomologica Argentina 68(1-2): 1-9.

Campos-Soldini MP (2011) A new species group of the genus Epicauta Dejean of southern South America, the bella Group (Coleoptera: Meloidae). Neotropical Entomology 40: 475-486. 
Campos-Soldini MP, Roig-Juñent SA (2011) Redefinition of the vittata species group of Epicauta Dejean (1834) (Coleoptera: Meloidae) and taxonomic revision of the species from southern South America. Zootaxa 2824: 21-43.

Campos-Soldini MP, Roig-Juñent SA (2015) Phylogenetic analysis and redefinition of the maculata species group of Epicauta (Meloidae: Meloinae: Epicautini). Insect Systematics and Evolution 46: 431-470. https://doi.org/10.1163/1876312X-45032126

Capinera J (2003) Striped Blister Beetle, Epicauta vittata (Fabricius) (Coleoptera: Meloidae). University of Florida, 1-4.

Denier PCL (1933a) Contribución al estudio de los melóidos americanos. Revista Chilena de Historia Natural 37: 237-246.

Denier PCL (1933b) Documentos para la clasificación de los meloidos americanos. Boletín del Ministerio de Agricultura de la Nación (Buenos Aires) 32(1): 35-52.

Denier PCL (1934) Cinco especies nuevas de meloideos sudamericanos. Revisat de la Sociedad Entomológica Argentina 6: 268-275.

Denier PCL (1935a) Coleopterorum americanorum familiae Meloidarum enumeratio synonymica. Revisat de la Sociedad Entomológica Argentina 1: 15-28.

Denier PCL (1935b) Coleopterorum americanorum familiae meloidarum. Enumeratio synonymica. Revisat de la Sociedad Entomológica Argentina 7: 139-176.

Denier PCL (1940) Enumeratio coleopterorum americanorum familiae Meloidarum. Corrigenda et addenda. Revista de la Sociedad Entomológica Argentina 10: 418-425.

Di Iorio OR (2004) Meloidae. In: Cordo HA, Logarzo G, Braun K, Di Iorio O (Directores) Catálogo de insectos fitófagos de la Argentina y sus plantas asociadas. Revista de la Sociedad Entomológica Argentina 2004: 97-101, 104-108, 115-121.

García-París M, Buckley D, Parra-Olea G (2007) Catálogo taxonómico-geográfico de los Coleópteros de la familia Meloidae de México. Graellsia 63(2): 165-258. https://doi. org/10.3989/graellsia.2007.v63.i2.92

García-París M, Ruiz JL (2013) Description of a new species of Epicauta (Coleoptera: Meloidae) from Mexico and comments on the Epicautacupraeola species-group. Graellsia 69: 57-77. https://doi.org/10.3989/graellsia.2013.v69.079

García-París MA, Ruizb JL, Sánchez-Vialascy A, López EK (2016) Una especie nueva de Epicauta de Venezuela y comentarios sobre el grupode especies de Epicauta (E.) vittata (Coleoptera: Meloidae). Revista Mexicana de Biodiversidad 87: 944-955. https://doi. org/10.1016/j.rmb.2016.07.016

Hayward KJ (1942) Primera lista de insectos tucumanos perjudiciales. Revista Industrial y Agrícola de Tucumán 42: 1-110.

Hayward KJ (1960) Insectos tucumanos perjudiciales. Revista Industrial y Agrícola de Tucumán 42: $1-144$.

Horn GH (1873) Revision of the species of several genera of Meloidae of the United Stated. Proceedings of the American Philosophical Society 13: 88-117.

Kaszab Z (1959) Phylogenetische Beziehungen des Flügelgeäders der Meloiden (Coleoptera), nebst Beschreibung neuer Gattungen und Arten. Acta Zoologica Academidae Scientiarum Hungaricae 5: 67-114. 
Kaszab Z (1960) Beiträge zur Kenntnis der Meloiden von Peru (Coleoptera). Studia Entomologica 3: 401-416.

Kaszab Z (1963a) Studien über Meloiden (Coleoptera). Annales Historico-Naturales Musei Nationalis Hungarici 55(Pars Zoologica): 335-346.

Kaszab Z (1963b) Merkmale der Adaptation, Spezialisation, Konvergenz, Korrelation und Progression bei den Meloiden (Coleoptera). Acta Zoologica Academidae Scientiarum Hungaricae 9: 137-175.

MacSwain JW (1956) A classification of the first instar larvae of the Meloidae (Coleoptera). University of California Publication in Entomology 12: 1-182.

Martínez A (1951) Notas sobre Meloidae. Una nueva especie del género Pseudomeloe F. et G. Comun. Instituto Nacional de Investigación de las Ciencias Naturales 2(7): 97-101.

Martínez A (1952) Notas sobre Meloidae II. Annales de la Sociedad Científica Argentina 153: 254-258.

Martínez A (1954) Notas sobre Meloidae III. Revista de Investigaciones Agropecuarias 8(3): 285-288.

Martínez A (1955) Notas sobre Meloidae IV. Una nueva especie y un nuevo nombre de Epicautas bolivianas (Col. Meloidae). Neotropica 1: 55-58.

Martínez A (1958) Notas sobre Meloidae (Col.) V. Neotropica 4(15): 77-80.

Martínez A (1959) Notas sobre Meloidae VI. Dos nuevas especies de Zonitis de Bolivia. Acta Zoolgica Lilloana 17: 139-148.

Martínez A (1992) Los Meloidae de Salta, Argentina (Coleoptera). Insecta Mundi 6: 1-12.

Pic M (1928) Divers coléoptères nouveaux de la République Argentine. Revista de la Sociedad Argentina de Entomología 2: 99-102.

Pinto JD (1975) The taxonomy of three species of maculate Epicauta (Coleptera: Meloidae), with descriptions of courtship behavior and first instar larvae. Journal of the Kansas Entomology Society 48: 429-452.

Pinto JD (1980) Behavior and taxonomy of the Epicauta maculata Group (Coleoptera: Meloidae). University of California Publication in Entomology 89: 1-111.

Pinto JD (1984) New generic synonymies in the Epicautina (Coleoptera: Meloidae: Meloinae). Proceeding of the Entomological Society of Washington 86: 378-382.

Pinto JD (1991) The taxonomy of North American Epicauta (Coleoptera: Meloidae) with a revision of the nominate subgenus and a survey of courtship behavior. University of California Publications in Entomology 110: 435.

Pinto JD, Bologna MA (2016) Beetles (Coleoptera) of Peru. Survey of the Families. Meloidae Gyllenhal, 1810. Journal of the Kansas Entomological Society 89(2): 202-209. https:// doi.org/10.2317/JKES151211.1

Pregill GK (1981) An appraisal of the vicariance hypothesis of Caribbean biogeography and its application toWest Indian terrestrial vertebrate. Systematic Zoology 30: 147-155 https:// doi.org/10.2307/2992413

Selander RB (1981a) The Custica group of the genus Epicauta (Coleoptera: Meloidae). Proceedings of the Entomological Society of Washington 83: 573-591. https://biodiversitylibrary.org/page/16364922 
Selander RB (1981b) Evidence for a third larval prey in blister beetles (Coleoptera: Meloidae). Journal of the Kansas Entomology Soc. 54: 757-783.

Selander RB (1982) Sexual behaviors, bionomics, and first instar larvae of the Lauta and Diversicornis groups of Epicauta (Coleoptera: Meloidae). Proceeding of Entomological Society of Washington 84: 797-821.

Selander RB (1984) The blister beetles of Florida (Coleoptera: Meloidae). Florida Department of Agriculture and Consumer Services, 4 pp.

Selander RB (1991) On the nomenclature and classification of the Meloidae (Coleoptera). Insecta Mundi 5: 65-94.

Selander RB, Mathieu JM (1969) Ecology, behavior, and adult anatomy of the Albida Group of the genus Epicauta (Coleoptera: Meloidae). Illinois Biol. Monographs 41: 1-168.

Selander RB, Weddle RC (1969) The ontogeny of blister beetles (Coleoptera, Meloidae). II. The effects of age of triungulin larvae at feeding and temperature on development in Epicauta segmenta. Annals of the Entomological Society of America 62: 27-39. https://doi. org/10.1093/aesa/62.1.27

Werner FG (1945) Some North American species of Epicauta (Coleop., Meloidae). Pysche 40: $65-73$.

Werner FG (1954) A review of the subgenus Gnathospasta of the genus Epicauta (Meloidae). The Coleopterists' Bulletin 8: 25-27.

Werner FG (1955) Studies in the genus Epicauta of the North American continent Coleoptera, Meloidae). I. Caviceps group. Bulletin Brooklyn Entomological Society 50: 1-12.

Werner FG (1957a) A new species of Epicauta from the Gulf Coast of Texas. Proceeding Entomology Society of Washington 59: 97-98.

Werner FG (1957b) Epicauta dugesi a valid species (Coleoptera: Meloidae) Psyche: 107-108. https://doi.org/10.1155/1957/41286

Werner FG (1958) Studies in the genus Epicauta of the North American continent (Meloidae) II. The Uniforma-Group. Coleoptera Bulletin: 1-19.

Werner FG (1973) Three new Epicauta from Mexico (Coleoptera: Meloidae). Proceeding Entomology Society of Washington 74: 458-463. 